$$
\text { bug, }+2 \text { cap. }
$$

Dite

\# 0520 Fterodroma externa May 24,1965 $11055^{\circ} \mathrm{N} \quad 148^{\circ} 00^{\prime} \mathrm{W}$

\#1051. Bulweria Bulwerii Mag 4,1965 $20^{\circ} 52^{\prime} \mathrm{N} \quad 157^{\circ} 28^{\prime} \mathrm{W}$

\#1052 Sterna fuscata May 5,1965

$$
18^{\circ} 33^{\prime} \mathrm{N} \quad 15^{\circ} 38^{\prime} \mathrm{W}
$$

A 1053 Sterocrarius longicauda May 7,1965

$$
13^{\circ} 20^{\prime} \mathrm{N} 15,011^{\prime} \mathrm{W}
$$

* 1054 Sula dectylatra May 8,1965

$10^{\circ} 5^{\circ} \mathrm{N} \quad 149^{\circ} 15^{\prime} \mathrm{W}$

\$ 1056 Pterodroma externa hay 8,1965 $10^{\circ} 42^{\prime} \mathrm{N} 149^{\circ} / 3^{\prime} \mathrm{W}$

\#1057 Sula dactylatra May 8,1965 $10^{\circ} 42^{\prime} \mathrm{N} \quad 149^{\circ} 12^{\prime} \mathrm{W}$

\#1058 Pterodroma externa May 8,1965

\#1059

$10^{\circ} 37^{\prime} \mathrm{N} \quad 149^{\circ} 16^{\prime} \mathrm{W}$

May \&, 1965

$10^{\circ} 35^{\circ} \mathrm{N} \cdot 149^{\circ} 15^{\prime} \mathrm{W}$

\# 1060 Sula dactylatra Muy 8,1965

$10^{\circ} 36^{\prime} \mathrm{N} \quad 149^{\circ} 14^{\prime} \mathrm{W}$

\# 1061 Pterodromo externa May 8, 1965 $10^{\circ} 35^{\prime} \mathrm{N} 149^{\circ} 20^{\prime} \mathrm{W}$

\#1062 Puffinus pacticus May \&,1965 $10^{\circ} 33 \mathrm{~N}-149^{\circ} 21^{\prime} \mathrm{W}$ 
1063 Patannes pariksus May, 8,1965

$$
10^{\circ} 3,4 N-149^{\circ} 23^{\prime} \mathrm{W}
$$

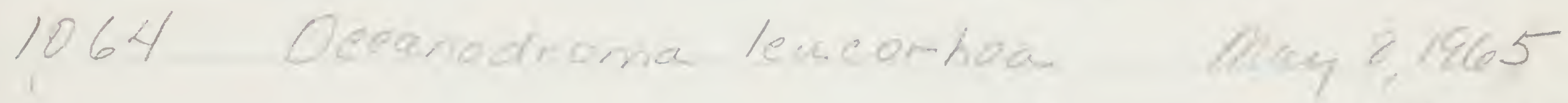

$$
10^{\circ} 24^{\prime} \mathrm{N} \quad 145^{\circ} 29^{\prime} \mathrm{W}
$$

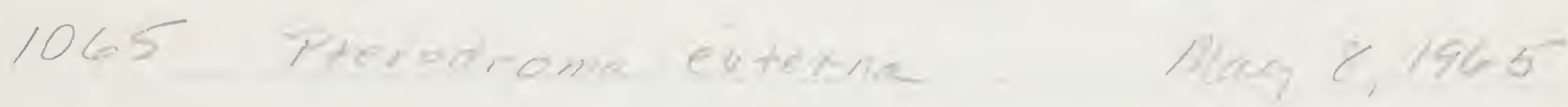

$10^{\circ}$ Q $3^{\circ} \mathrm{N} / 49^{\circ} 46^{\prime} \mathrm{W}$

1066

1920, 8, 1965

$09^{\circ} 40^{\circ} \mathrm{N} \quad 100^{\circ} 12^{\circ} \mathrm{W}$

1068 Sala dactoletion

$\operatorname{lin} 10,9,1865$

1069 Strome fiscata

$08^{\circ} 06 \mathrm{~N} 15,047^{\prime} \mathrm{W}$

Mary 9,1965

O8.01'N 150000'W

1070

Aitcay 21965

OS"0.'N $152^{\circ}$ Do'h

1071 Fandousa parficus

1hary, 9,1965-

C8OONN $152^{\circ}$ OL'W

1072 Phanderma externa

Way, 9.1965

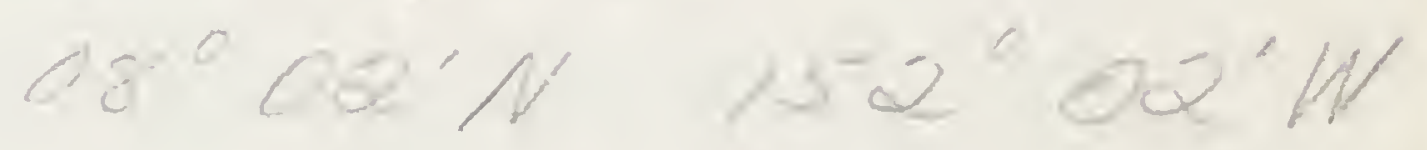

1073 Sremen -2050mo

$10149,9,1865$

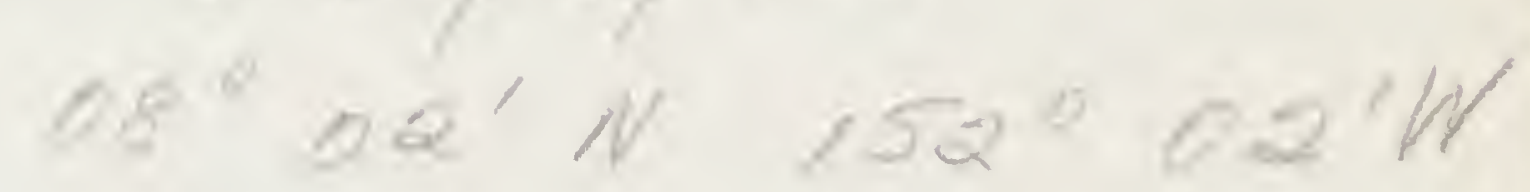

1074 Stena fuscara

$110.89,865$

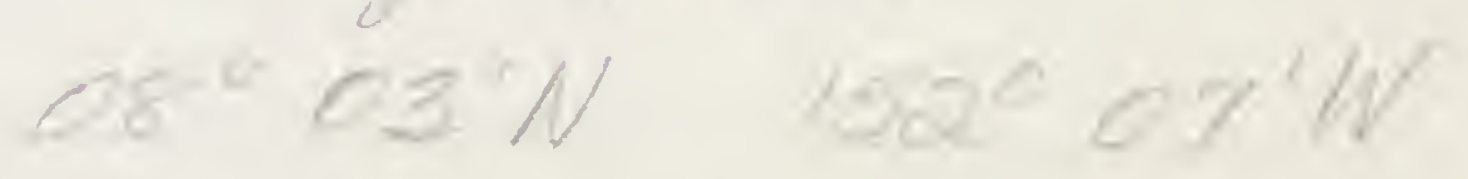

1075

120.91965

$08^{\circ} 03^{\circ} \mathrm{N} \quad 152^{\circ} 05^{\circ} \mathrm{W}$ 
1077 Sala Giectolatioa

2079

1080 Prevodroma externa

108, Swa dactylatrat

1082 Sheme fuswor

1083 Sula diectolatra

1085

1086 Ptempdima exterma

108n. Stemcorarms longicauda

1088 Sula dactylatra

1089 Stema Reremtar
$190,4,196=$

$O 8^{\circ} 0^{\circ} \mathrm{W} \quad 152^{\circ} 45^{\prime} \mathrm{W}$

1100 a 18 . 1965

$09034 \mathrm{~K} \quad 15,053 \mathrm{~W}$

miay 10,1965

$09^{\circ} 35 N^{\circ} \quad 137^{\circ} 57^{\prime h}$

the 10,1965

$69^{\circ} 35{ }^{\circ} \mathrm{N} \quad 153^{\circ} 05^{\prime W}$

Mlay 11, 1965

$08^{\circ} 05^{\prime \prime N} \quad 155^{\circ} 46^{\prime} \mathrm{W}$

Mony 11, Nacs

$28^{\circ} 49^{\prime N} \quad 155^{\circ} 216^{\prime W}$

Mreg, - 11, 1985

$08^{\circ} 43 \mathrm{~N}-153^{\circ} \mathrm{5} 0^{\circ} \mathrm{W}$

N11ay 11. 1965

$05^{\circ} 43 \mathrm{~N} \quad 155^{\circ} 50^{\circ} \mathrm{W}$

1iley 114,1965

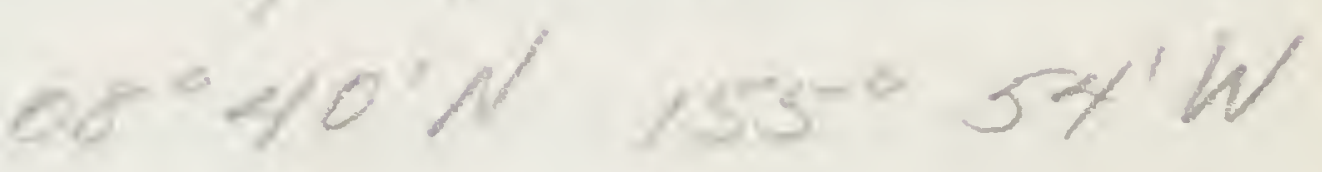

May 11, 1965

$08^{\circ} 36^{\prime} N \quad 15^{\circ} 59^{\prime} W$

Theray 11,1965

$\cos ^{\circ} 33^{\circ} \mathrm{N} \quad 156^{\circ} \mathrm{M} / \mathrm{W}$

Nixy 11,1965

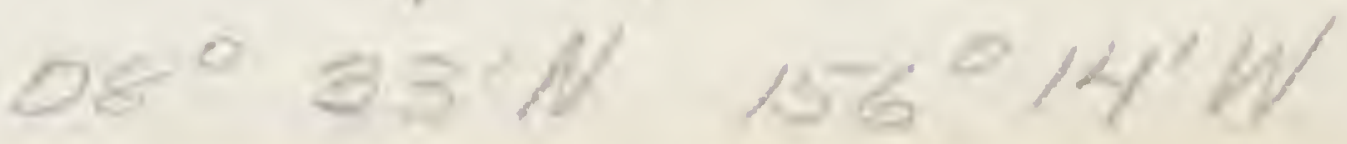


logi sula dartylatra

1092 Stema fustata

1093 Tuffinces notw watus

1094 Sala dactylatra

1045

1096

1098

1099

1100

110) Stema fascata

1102 Fafonas partieus.

1103 Sula dactrlatra
Thar, 11,1965

$08^{\circ} 29^{\circ} \mathrm{N} \quad 156^{\circ} 25^{\circ} \mathrm{W}$

IItiay 11, 1965

$21^{\circ} 27^{\prime} \mathrm{N} \quad 156^{\circ} 29^{\prime} \mathrm{W}$

Alay 11,1965

$08^{\circ} 18 \mathrm{~N}^{\circ} 156^{\circ} 4 \mathrm{I}^{\prime} \mathrm{W}$

1Hain 11, 1965

$08^{\circ}, 16^{\prime N} 156^{\circ} 43^{\prime W}$

Thay 11,1965

$08^{\circ} 12^{\circ} \mathrm{N} \cdot 156^{\circ} 46^{\prime} \mathrm{W}$

Mhay II, 1465

$08^{\circ} \times 11=156^{\circ} 416^{\prime} \mathrm{W}$

Miay. 11, 1865

$12^{\circ} \mathrm{N} \quad 156^{\circ} 46^{\circ} \mathrm{h}$

Alay 11, 1965

$08^{\circ} 04^{\circ} \mathrm{N} \quad 156^{\circ} 55^{\prime} \mathrm{W}$

Ilikey $11,1965^{2}$

$08^{\circ} 0371157^{\circ} 07^{\circ} \mathrm{h}$

Marg 11,1965

Q6 $03 \mathrm{~N}^{\circ} 152^{\circ} 02^{\circ} \mathrm{W}$

Nian 11, 1965

$08^{\circ} 03 N 157^{\circ} 07^{\prime} \mathrm{W}$

livey 11, 1965

Q $0^{\circ} 08^{\circ} \mathrm{N} \quad 15^{\circ} 7^{\circ} 08^{\circ} \mathrm{W}$ 
1104 Sula dactylatra

1106 Germa fúscate

1107 Sula dactylatra.

1109

Mo Paffores panficus

III) Eala danolplatra

wa2 7raethon. lepturus

II3 Sterna fuscara

$1 / 15$

Whe Phacthori leptarios

1143 Srema fersenta

1144
Man 11, 1965

$08^{\circ} 05^{\prime} \mathrm{N} 15^{\circ} 10^{\prime} \mathrm{W}$

MPQ 12,1965

$28^{\circ}$ NIN $160^{\circ} 01^{\prime} \mathrm{W}$

- Praey 12,1405

$08^{\circ} 15^{\circ} \mathrm{N} 160^{\circ} 0 \mathrm{H}^{\prime} \mathrm{W}$

Mlay 12, 1965

$08^{\circ} 13^{\prime} \mathrm{N} \quad 160^{\circ} 26^{\circ} \mathrm{W}$

Mary 12, 1965

$08^{\circ} 12$ N $160^{\circ} 34^{\prime} \mathrm{ll}$

May 12, 965

$08^{\circ} 12^{\circ} \mathrm{N} \quad 160^{\circ} 59^{\circ} \mathrm{W}$

May 14,1965

$1{ }^{\circ} 50^{\circ} \mathrm{N} 166^{\circ} 43^{\circ} \mathrm{W}$

Maey 15,196:

$13 \circ 32 \mathrm{~N} 169^{\circ} 45^{\prime} \mathrm{W}$

$111 \mathrm{ke}, 16,2965$

$13019^{\prime} \mathrm{N} 171^{\circ} 11^{\prime} \mathrm{W}$

Aliong 16,1965

$15055 \mathrm{~N} \quad 170^{\circ} 34 \mathrm{~W}$

Niney 21, 1965

$19^{\circ} 02^{\prime} \mathrm{N} \quad 120^{\circ} \mathrm{NI} \mathrm{W}$

Alag 21, 1465

$19^{\circ} 02^{\prime} 11 \quad 170^{\circ}$ " $1 \mathrm{~W}$ 
1149 Sterna furatio

Nineg 22, 1965

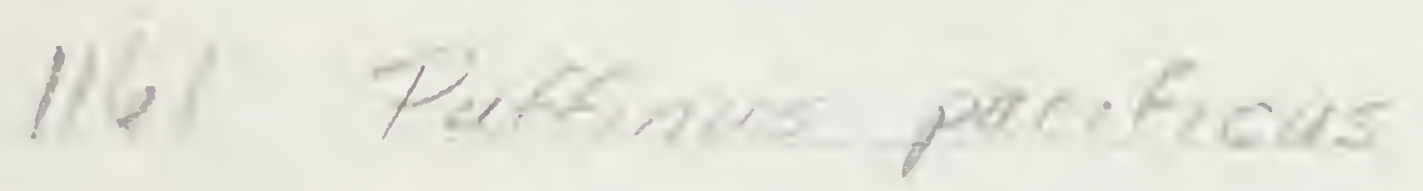

$20^{\circ} 59^{\mathrm{W}} 168^{\circ} \mathrm{OA} \mathrm{W}$

1162

$1172,23,1965$

$2,039 \mathrm{WL} \quad 164^{\circ} 38^{\circ} \mathrm{W}$

1164 Stremon fiescenta

May 23, 1965

$2)^{\circ} 33 N^{N} \quad 164^{\circ} 38^{\prime} \mathrm{W}$

1165

Diey, 23, 1965

2.033'N N164035'W

Nhey 23,1965

$21^{\circ} 33^{\prime} \mathrm{N} \quad 164^{\circ} 38^{\circ} \mathrm{W}$

1107 Pafsoms paoficus

Mey, 23,1965

1169 Sterna fiscata

$2,033 \mathrm{~N} 1644^{\circ} 30^{\prime} \mathrm{W}$

Plag. $23,16 \mathrm{~s}^{\circ}$

1171 Fufters pecifieus

2.03317 164 $38^{\prime W}$

Hay 23,1965-

$205311164^{\circ} 38^{\prime W}$

1172

thay $23,183^{\circ}$

$21^{\circ} 33$ N $164035^{\circ} W$

1173

thay 23, 1965

$21^{\circ} 33=11 \quad 1644^{\circ} 38^{\prime} W$

1175 Gxy13 alba

Mocy 23,1965

2. 33 M $164^{\circ} 35^{\prime} \mathrm{W}$

1182 Paffinas parficas

Alang $23,196=$

$21^{\circ} 34 \mathrm{~N} \quad 164^{\circ} 37^{\prime} \mathrm{W}$ 
1184 Sterna fiscotor

1186 Gygis alba

1187 Sterna lunata

1182 Fuffinds pacificus

1199

1190

1191 Buluera bulwerii
Ma, 231965

$2,366^{\circ}$ II $124^{\circ} 35^{\circ} \mathrm{W}$

Hiacy 23,1963'

2, $38^{\circ} \mathrm{N} 164^{\circ} 06^{\prime} \mathrm{W}$

Dlecy, 24,1965

$2,042 \mathrm{~N} N 161^{\circ} 50^{\prime} \mathrm{W}$

Ma4 $24,1965^{\circ}$

$2,042 \mathrm{~N} \quad 161^{\circ} 40^{\circ} \mathrm{W}$

Mnen 24, 1965

$21^{\circ} 43 \mathrm{~N} \quad 161^{\circ} 22^{\prime} \mathrm{W}$

May 24, 1965

$2,043 \mathrm{~N} \quad 161^{\circ} 22^{\prime} \mathrm{W}$

Hoy, 24,1965

$21^{\circ} 43^{\prime} \mathrm{N}-161^{\circ} 17^{\prime} \mathrm{W}$

Nhay 24, 1965

$21^{\circ} 44^{\circ} \mathrm{N} \quad 161^{\circ} 12^{\prime} \mathrm{W}$

Alecy 24,1965

$210441 \mathrm{~N} \quad 161^{\circ} 11 ' \mathrm{~W}$

thay $24,1965^{-}$

$21^{\circ}$ समI' N $161^{\circ} \mathrm{II} W$

Aleay 24,1965

$2.044^{\prime} \mathrm{NI} 161^{\circ}$ 'W

Inicy 24,1465

$2,044, N \quad 161^{\circ} / 1 W$ 
1197 Bubveria bulweri

1198 Stemp fuscara

1199 Bulweria bulwem.

1200 steme ferrata

12ul Frocelsterna cerulea

1202 Farkenues partione

1003

1204

1205

1206

5238 Ducula mompica

5239 Zneda 7acifea
Nay 2.4, 1965

$21^{\circ} 444^{\prime N} 161^{\circ}$ o. $\mathrm{W}$

$1120,211,1465$

$\because 7^{\circ} 45 \mathrm{~N} \quad 160^{\circ} 5 \mathrm{Y}^{\prime} \mathrm{W}$

$1720,24,1965$

$20^{\circ} 45^{\circ} \mathbb{N} 160^{\circ} 541 \mathrm{~W}$

Ho. $241960^{\circ}$

$2,0^{\circ} y,-N \quad 160^{\circ} 48^{\prime} \mathrm{Wl}$

$2,040, \mathrm{~N} \quad 160^{\circ} 46 \mathrm{~W}$

$21^{\circ} 44^{-1}$ N $160^{\circ} 46^{\circ}$ 'W

May 24,1965

$2,45 \%$ N $160^{\circ} 46^{\prime} \mathrm{W}$

17log, 24,1965

$22^{\circ} 45^{\prime N} / 160^{\circ} 46^{\prime} \mathrm{W}$

May 24, 19les

2,045 IV $160^{\circ} 46$ W

2. $45 . \mathrm{N} \quad 160246 \mathrm{~W}$

Arataue Aloil, Tokelau Istand's Same 
5240 Ducula pacifica March 3, 1965 - Atafa Atoll, Toholeu Islands.

20262 Sterna fuscata. Aug 6,1964 Creen I, Kure Atoll

20274

Aug. 18, 1964

20279

Aug. 28,1964

20280

20284 Arenaria interpres Sept. 6,1964

20295 Pluvialis dominica Sept.15,1964

20309 Erolia melan, itos Sept. 25,1964

20310

20311

20318 ARENAMa interpres Sept 30, 1964

20323 " "Oet. 2,1964

20331 Pluvialis dominica Oet, Q,1964

30359 Pterodrama hypolenca Oct.24,1964

20363 Sula lenogaster

Oet 30,1964

20364 Anous Stolidus

Oet: 31,1964

20365 Pterodroma hypoleuca Nov 2,1964

20366 Limnodromus sp. Oet 30,1964 SnnD I. Mioway

20367 Heteroscelus incanam Oet30,1964

20369 Eroha melanotos Oet, 21,1964

20370

20371 Crreus cyaneus

Eastern I. Misuay

20373 Frequta minor

Nor. 3, 1964 Geeen I. Kure Atoll 
20375 ARENANIA intexpres

20379 Anas acuta

20380 Pluvialis dominica

20381 Sula Sula

20383 Larus sp.

20383 Mareca penelope

20403 Larus sp.

20405 Rissa tridactyla Dec, 30,1964

20414 Larus sp. Tan. 17,1965

20415 Larus argentatus Jan. 20,1965

20441 Diomedea niqripes Feb. 26,1965

20445 Oceanodroma marthami March1,1965"

20446 Faleo peregrinus

20447 Larus sp.

March 3,1965

20448 Larus occidentales Mard 8,1965

20449 Larus sp.

20450 Larus sp.

20452 Phacthon rubricauda March 8,1965

20453 Anas acuta March 17,1965

20454 "March 19,1965

20456 Gygis alba March 24,1965

20461 Lerus sp. April 1,1965

2509 Anous stolidus. Aug201964 Sand I. Johnston Atoll 
25029 Sterna fuscata

25034 Anous stolidus

25036 Sterna fuscata

25038

25039
Aug "1,1964 Sand I

Aug. 18, 1964 Sand I. Tohnston Atou

Aug. 15, 1964

Ang 25,1964 NorthI.

Sept.1,1964 Sand T

30036 Procelstema cervlea Oet, 17,1964 Marshall T.

Bikar Atoll Arumenis.

30079 Heteroscelus incanum Oet.22,1964 Marshall Fslaints

Tata I. Takn Atoll "!

30084 Sterna fuscata Oet.22,1964 Marshall I. Taks I. Takn Atoll

30099 Arenaria interpres Oet 24,1964 Marshall I.

Loj. I. Eritub Atoll

30103

3011. Stema sumatrana Oet.25,1964 Marshall Istands

Enego I. Eritub Atoll

30290 Heteroscelus incanum Nov.14,1964 Gibert Istands KotabuI. Makin Atoll

30292 Sterna sumatrana Nov.14,1964 Gibert Islands.

Sandbarzmi S. E. Tukureret. Makin Atoll

30293

Nov. 14,1964 Gibert Islands

Sandbar $\frac{1}{2}$ mi S.E. TukurereI. Makin Atoll

30295 Sterna sumatrana Nov, 14,1964 Gibert Islanks.

Sanbar Smi S.E. Tukureref. Makin Atoll 
30296 Sterna sumatrana Nov.14,1964 Gibert Islands Sandbar-2mi. S.E. Tukurece I. Makin Atoll $30297 "$ "date ' location Same 30313 Heteroscelus incanum Nov.14, 1964 Gibert Islands 110002 Butaritari I. Makin Atoll March 7,1965 Hawaii I. Laysan I.

110003 Pluvialis dominica Mard 3,1965 Laysan

110004

110005

110006 Lobipes lobatus

110008 Squatarda squatarda

110009

110015 Ereunetes mauri

110018 Calidris canutus

110019 Larus sp.

110021 Larus sp. bane

Bane"

'bance"

Mard 13,1965 Hawain I. Lisianski Island

Dame date + lecation March 15,1965 Hawaii I. Pearl + Hermes reef.

March 15,1965 Hawaili I.

Peart + Hernes Reef.

March 18,1965 Hawaic I.

Pearl + Hermes Beef.

March 12,1965 Hawain I.

Lisianski I. 



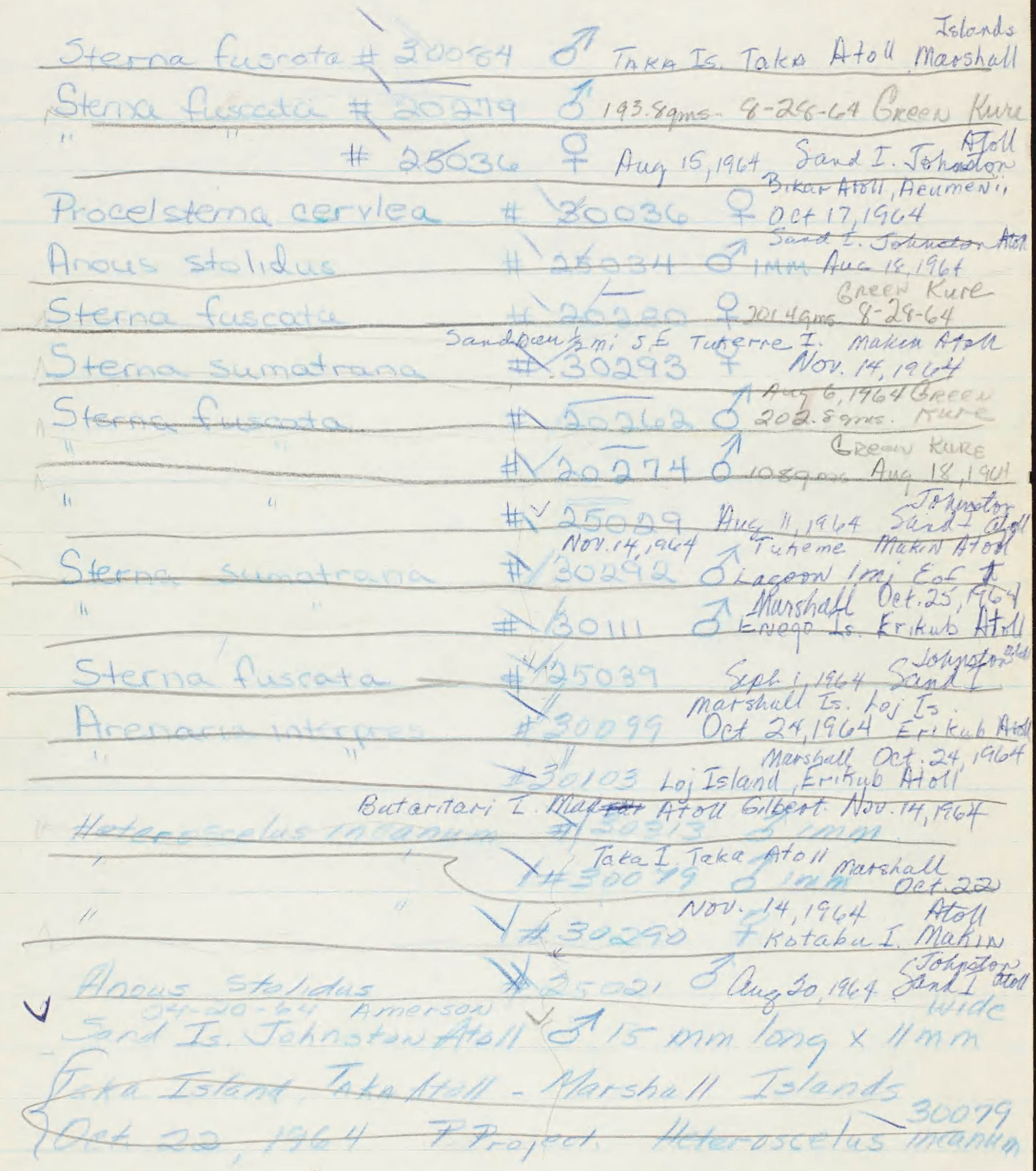




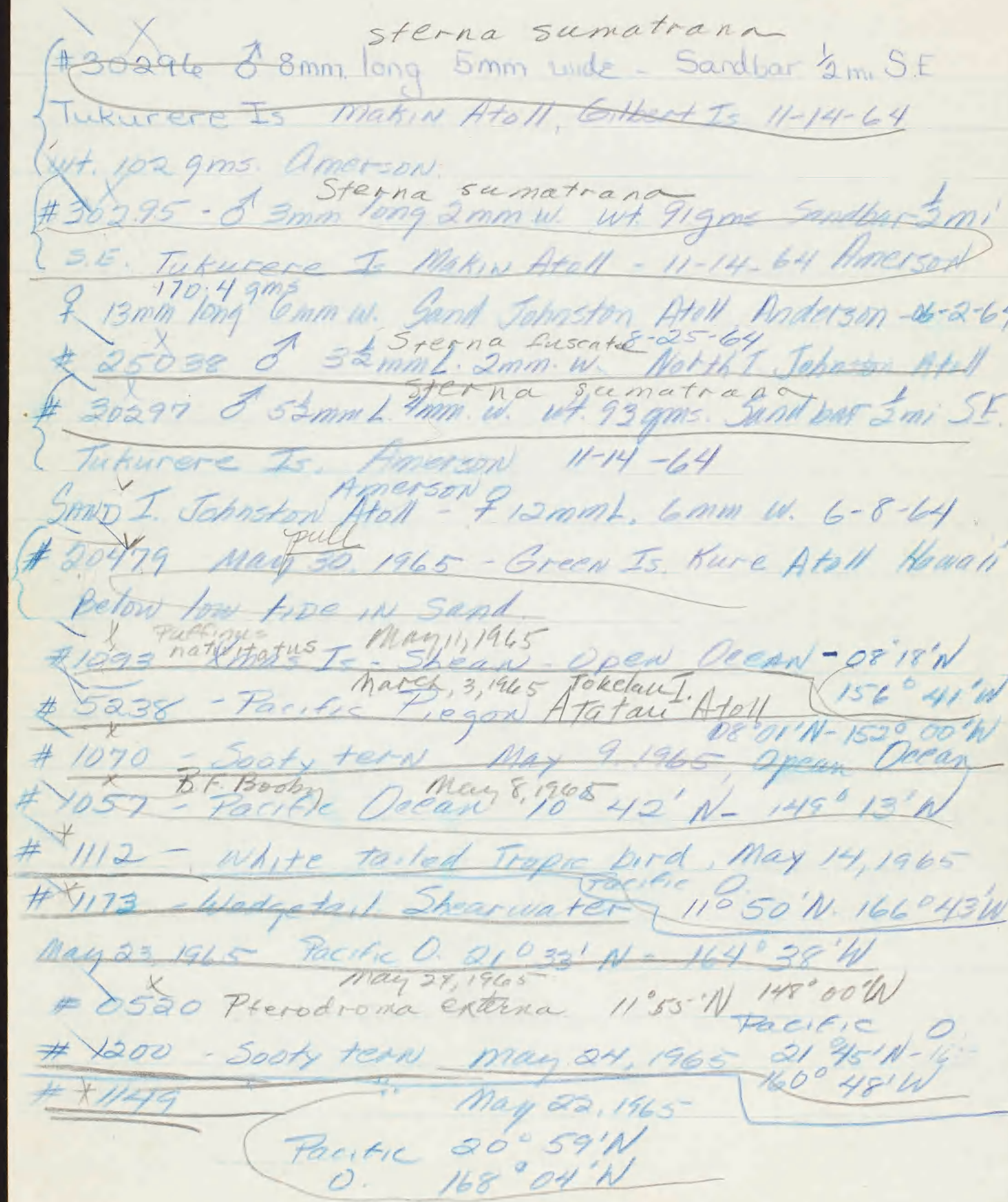


H1082 Mayll,1965 Soty

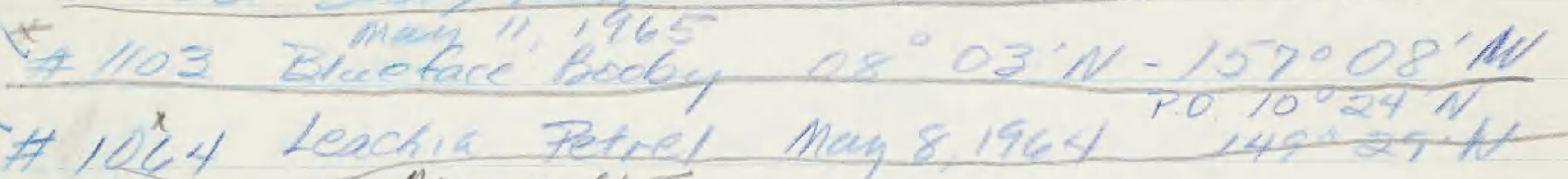

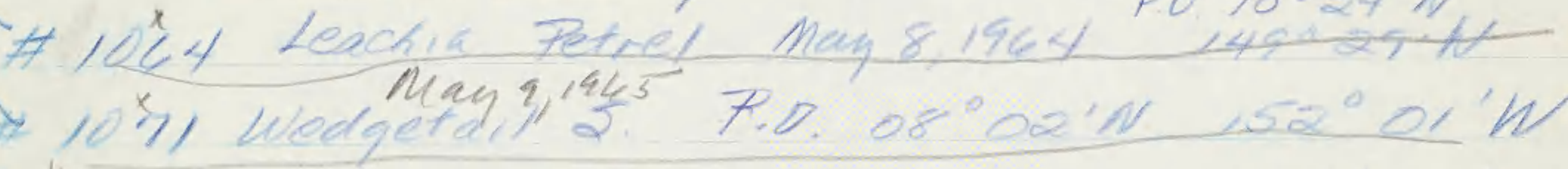

$+497$

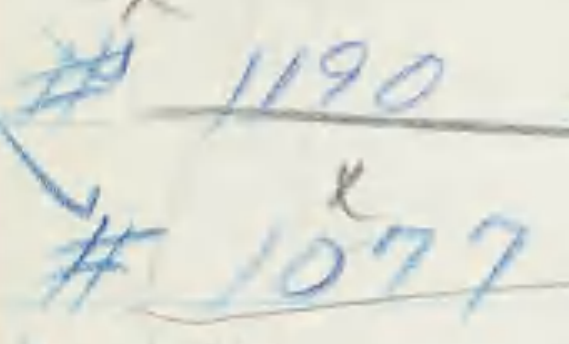

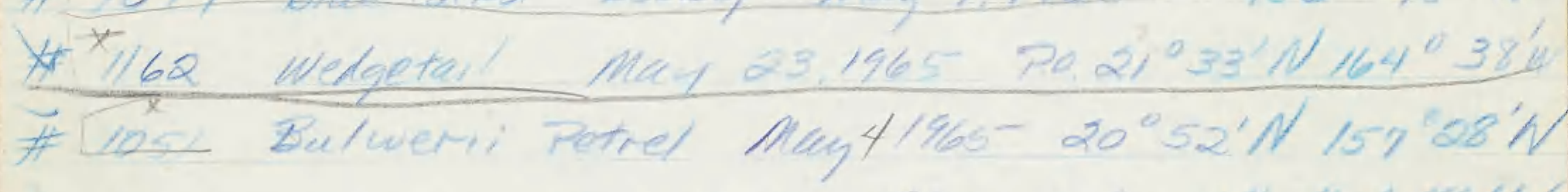

\& 1207

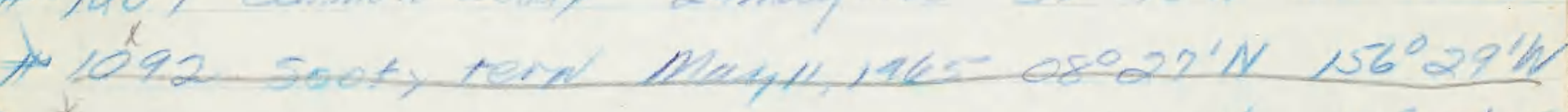

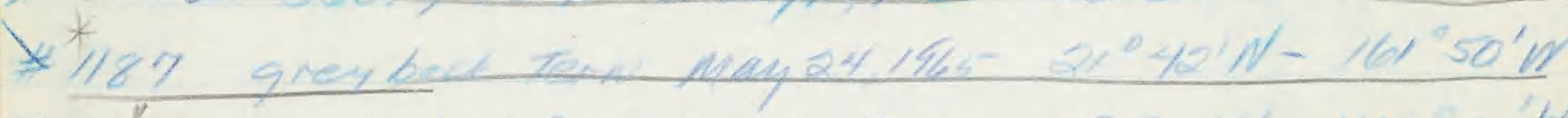

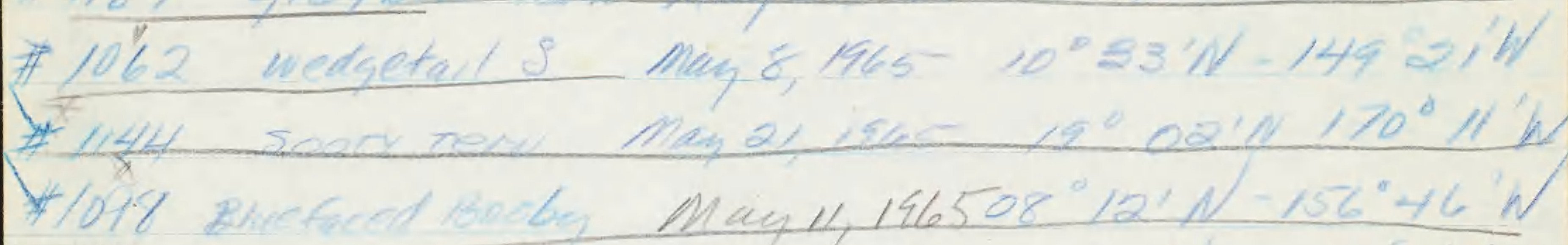

\#1096

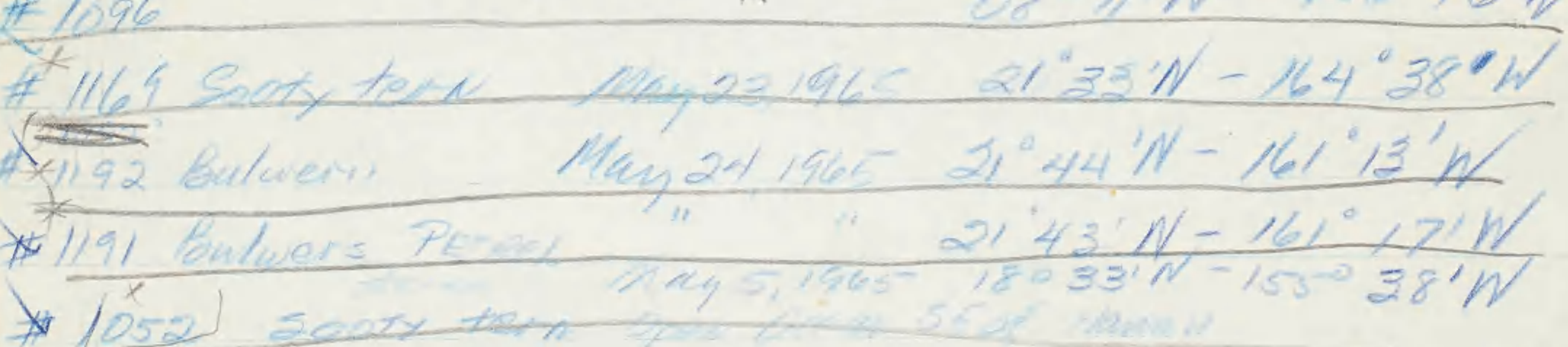

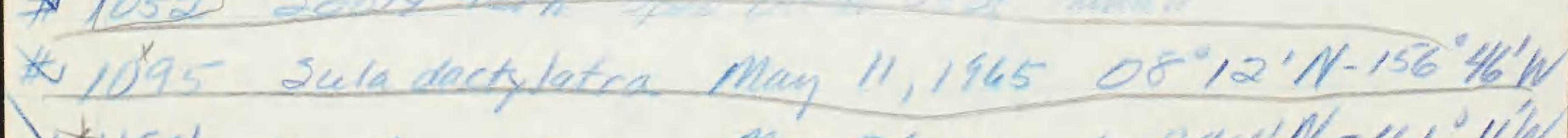

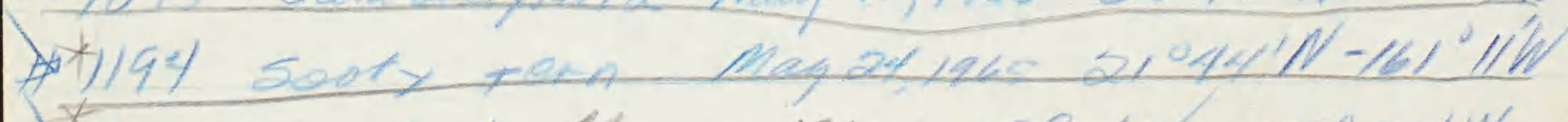

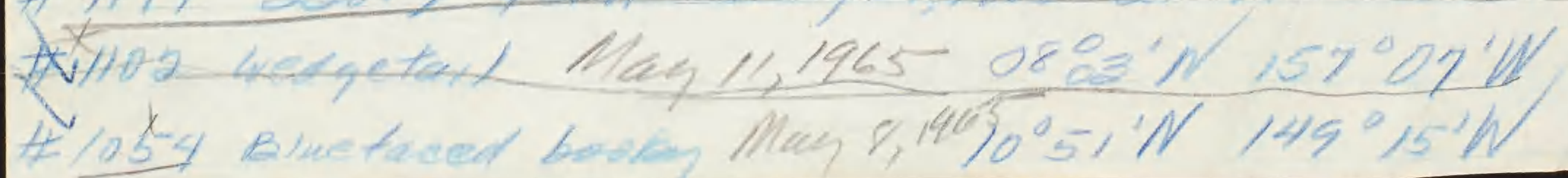




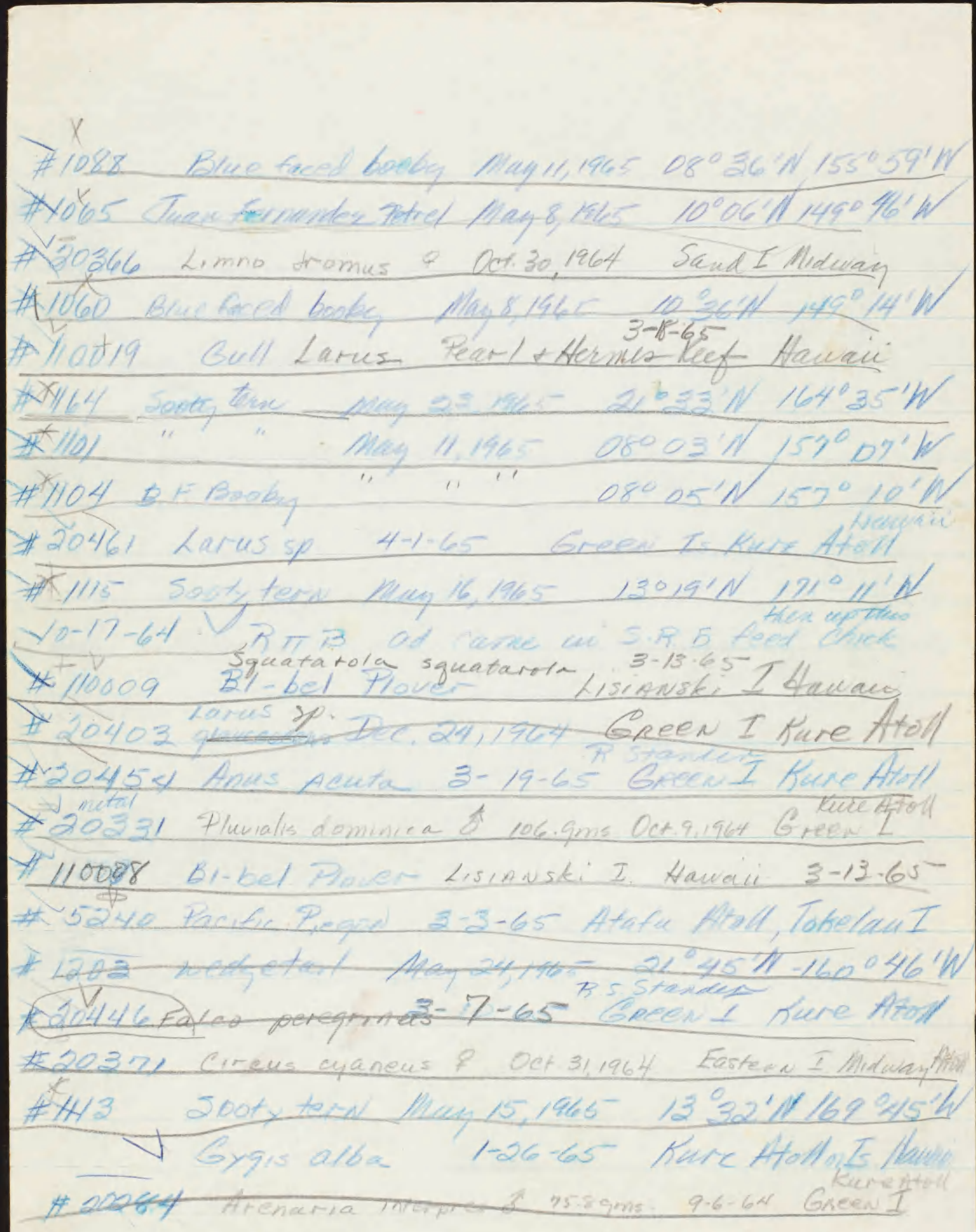




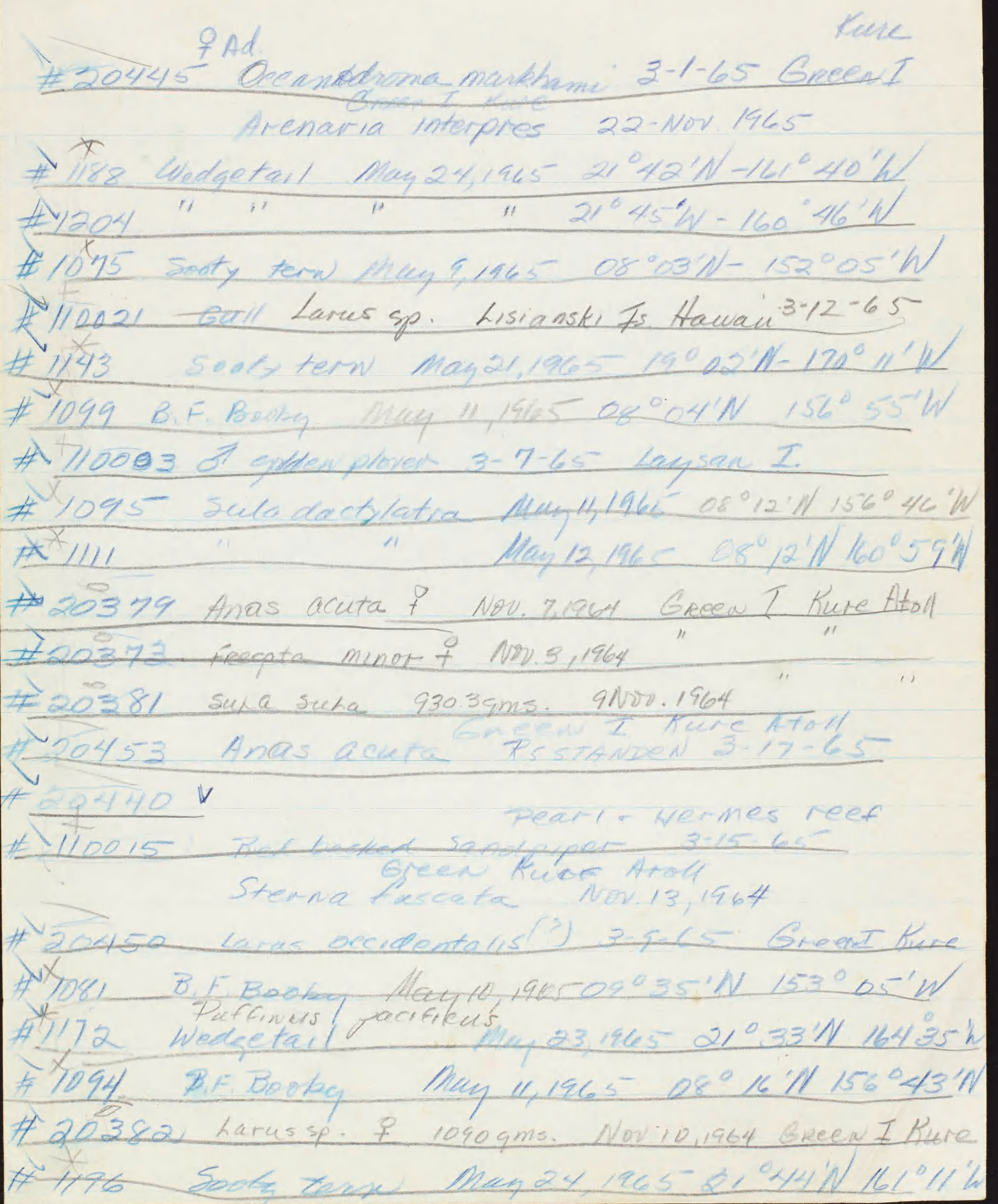


450447 Larns sp?' 3-7-1945 GReen I Rum Atoll

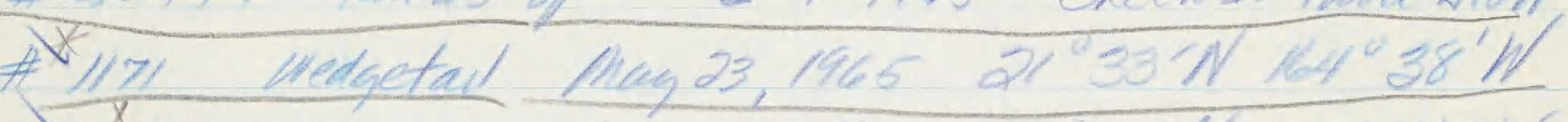

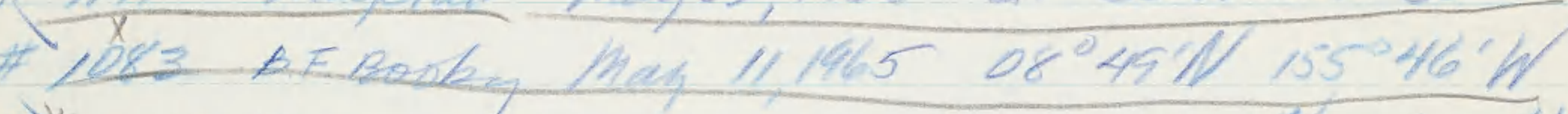

4*1199 Bulwers petrel noug24,1465 21045 II $160^{\circ} 541$

A 20995 Muvialis dommica of $97.3 \mathrm{gms}$. Sept 15,1964 Geeen I Kure

\# 20441 Dromeder nuqupes 26 Feb 1965 Geeen I Rue

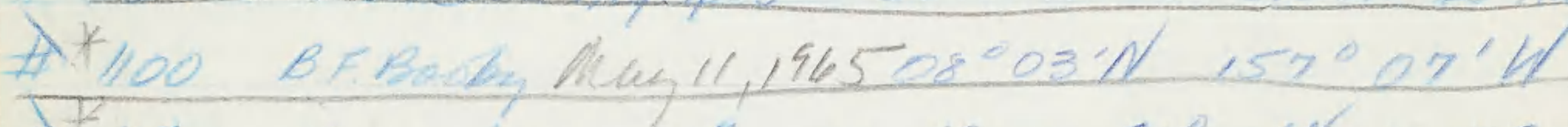

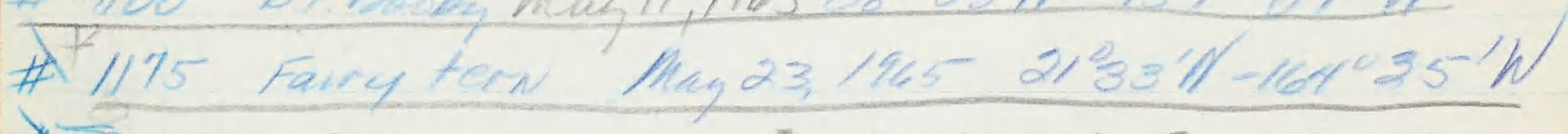

A 20370 Eroria melanotos of Oot 31,1964 Sand I Miduan

\# 110004 Coldew Dorar 3.7.65 Aawai Laysan I!

\#20310 krolia melanotos of 39.2 gms. Sept 25,196\% Green. I hure

\#20369 oct311964 Sand Mndway

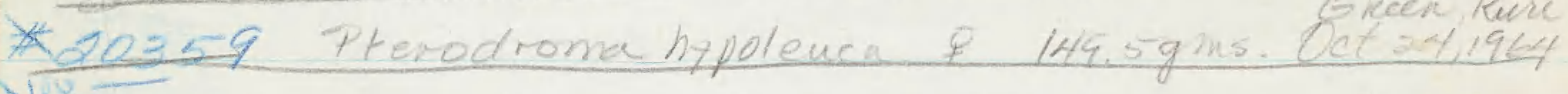

$\times 20282 T$

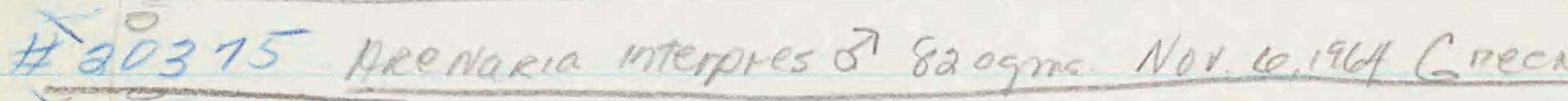

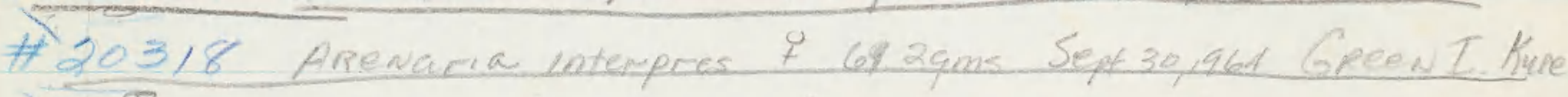

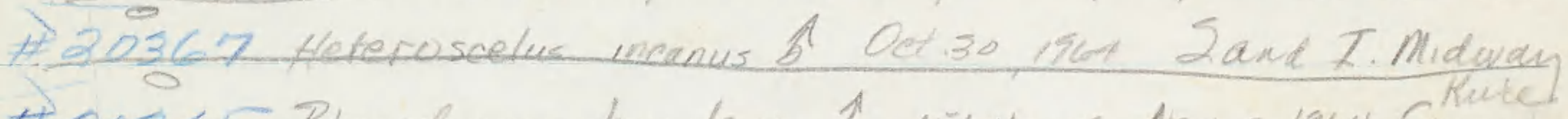

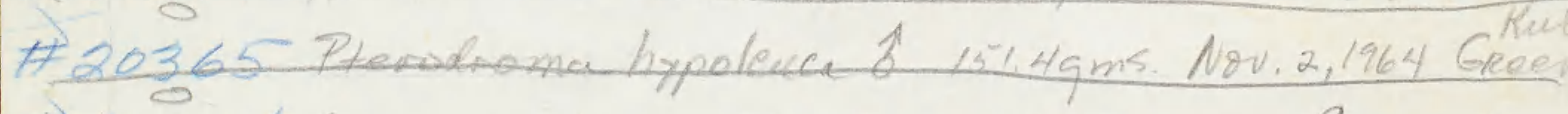

\# 20364 Anows stoldus $165.79 \mathrm{~ms}$. Oet 31,1964 Gnoed, Kive

20380 Plumalus domicuea of $106 \mathrm{gms}$. Nor 5, 1964

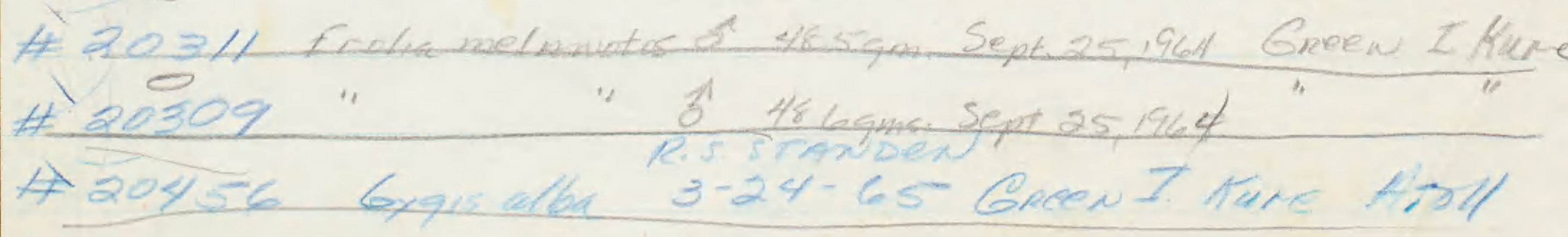




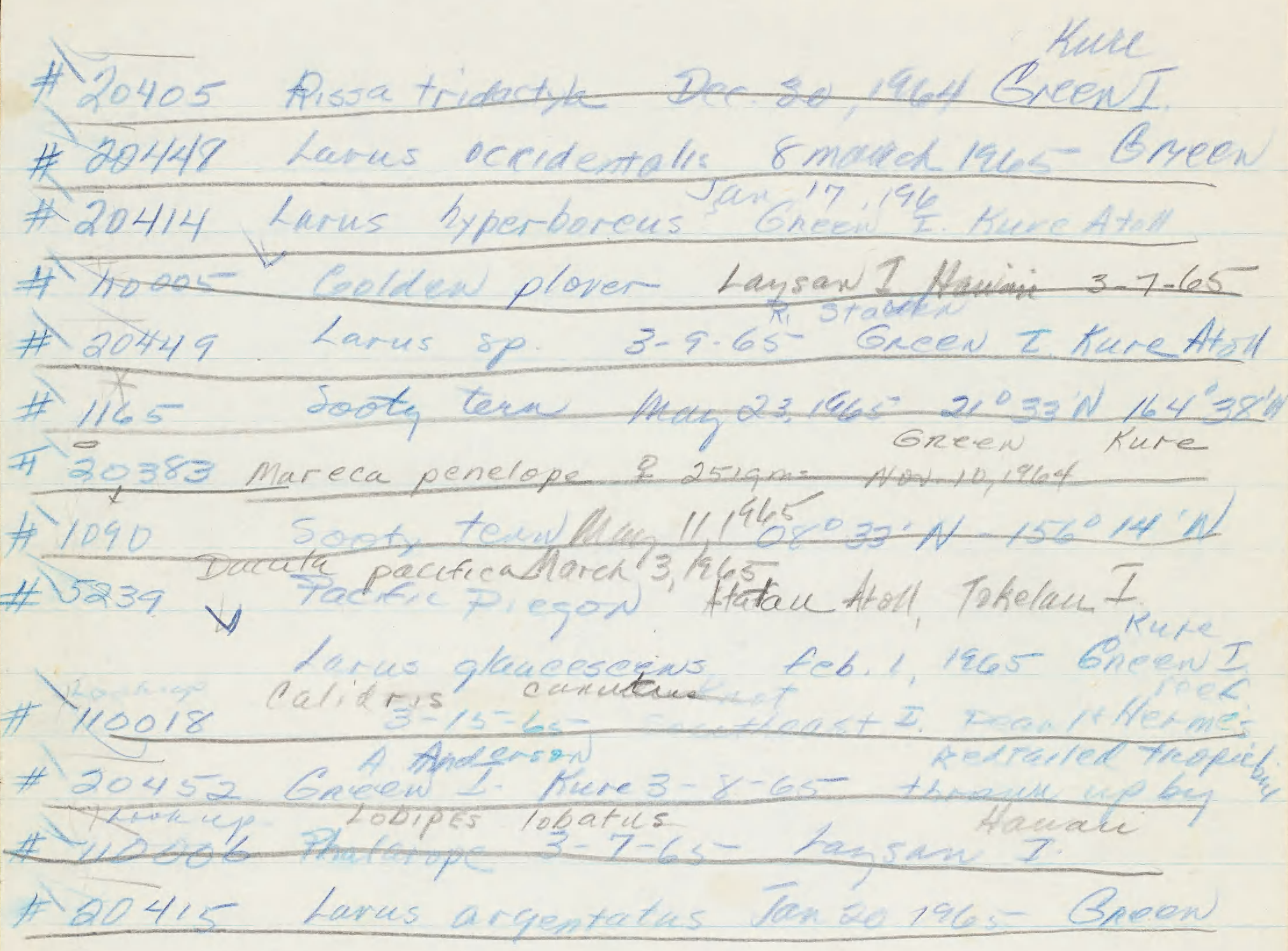




$\begin{array}{lll}20380 & 25034 & 110015 \\ 20381 & 25036 & 110018 \\ 20382 & 25038 & 110019 \\ 20383 & 25039 & 110021 \\ 20403 & 30036 & \\ 20405 & 30079 & \\ 20414 & 30084 & \\ 20415 & 30099 & \\ 20440 & 30103 \\ 20441 & 30111 & \\ 20445 & 20290 \\ 20446 & 30290 \\ 20447 & 30293 \\ 20448 & 30295 \\ 20449 & 20296 \\ 20450 & 30297 \\ 20452 & 30313 \\ 20453 & 110002 \\ 20454 & 110003 \\ 20456 & 110004 \\ 20461 & 110005 \\ 204149 & 110006 \\ 25021 & 110008 \\ 25029 & 110009\end{array}$


0520

\begin{tabular}{|c|c|c|c|c|}
\hline 1021 & 1077 & 1104 & 1184 & $\begin{array}{l}20262 \\
20574\end{array}$ \\
\hline 1052 & 1079 & 1106 & 1186 & $20=79$ \\
\hline$+05=$ & 1080 & 1107 & -1187 & 203280 \\
\hline 1054 & $10 E \%$ & 1109 & 1188 & $28020 \times 1$ \\
\hline 1256 & 1082 & $1 / 10)$ & 1199 & $5-284$ \\
\hline 10,7 & $108=$ & 1111 & $\cdot 1 / 720$ & 20295 \\
\hline $10-7$ & 1085 & 1112 & 117 & 20309 \\
\hline $10=8$ & 1086 & $1 / 1 / 3$ & 1192 & 20310 \\
\hline 1060 & 1087 & $1115^{-}$ & 1193 & 20311 \\
\hline ter & 1088 & 11166 & 2494 & 20318 \\
\hline 1062 & 10899 & 1143 & $1169=$ & 20323 \\
\hline $106 \div$ & $\cdot 370$ & $1 / 44$ & 1146 & $2+3 \geq 1$ \\
\hline 1064 & 1091 & $1 / 49$ & 1197 & 20355 \\
\hline $10 \leqslant 5$ & 1092 & $\forall(c)$ & 1198 & 20363 \\
\hline 1366 & $\angle 0,23$ & 11602 & 1199 & 20364 \\
\hline 10 Ext & 1094 & $1 / 10$ & 1200 & $2036=$ \\
\hline 1067 & 1095 & $116=$ & 1201 & 201366 \\
\hline 1020 & 2086 & 1167 & 1202 & 201667 \\
\hline 1071 & 1092 & 1169 & 1203 & 20367 \\
\hline $10 \times 3 x$ & 1099 & 1171 & -1204 & 20770 \\
\hline $1072=$ & $11 \theta 0$ & $1172^{2}$ & $120+$ & 2037 \\
\hline 1074 & 1101 & 1173 & 1206 & 10120373 \\
\hline 1075 & 11021 & 1175 & 5238 & 20372 \\
\hline $107=$ & 1103 & $11 / 0$ & $\begin{array}{l}5239 \\
5240\end{array}$ & $2037 x$ \\
\hline
\end{tabular}




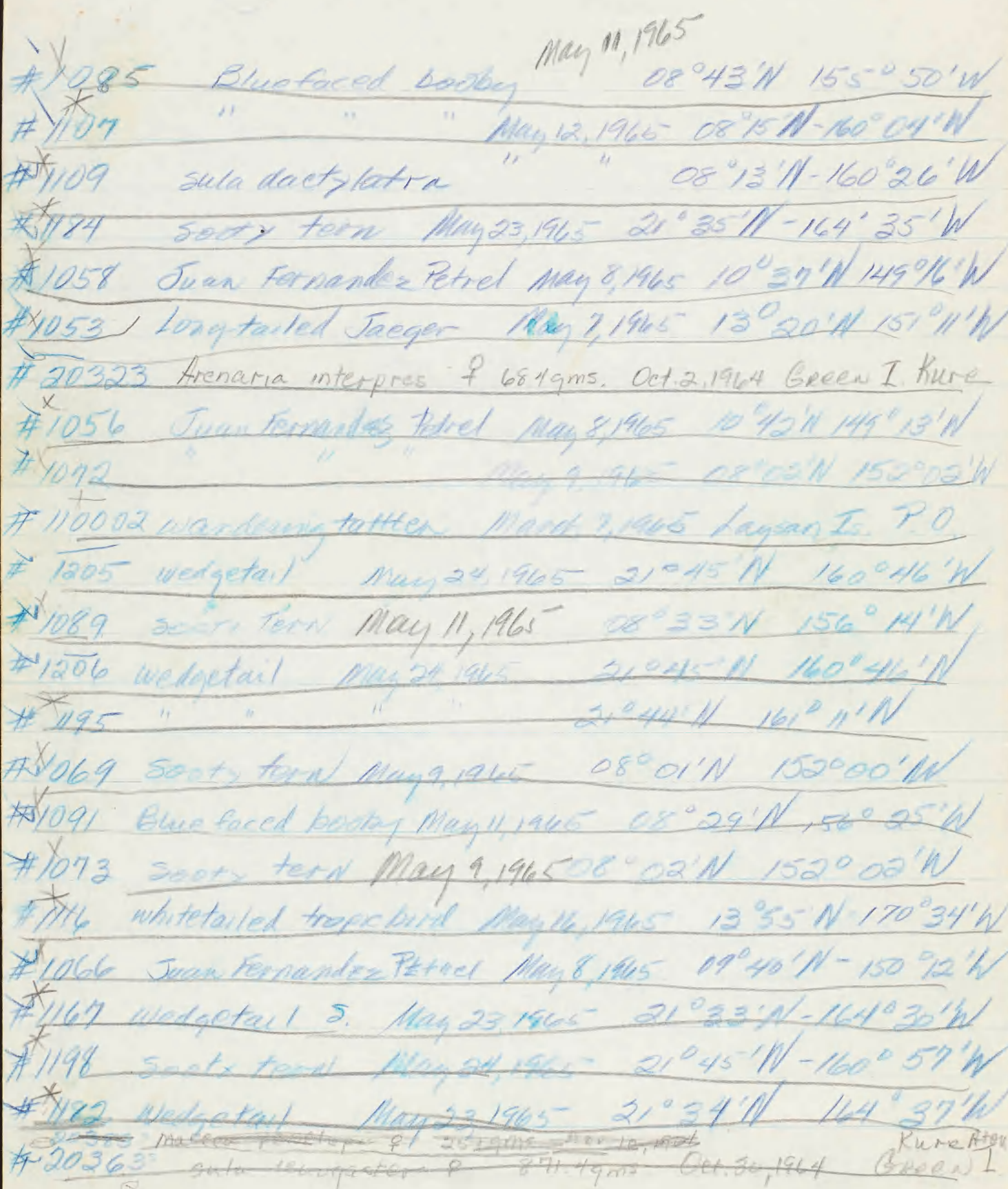


LOYSAN Albatrosa

195150378 Nowell's Stresrustor

10520271

$1713026 ?$

Stompetes

LNA 344

NH 362

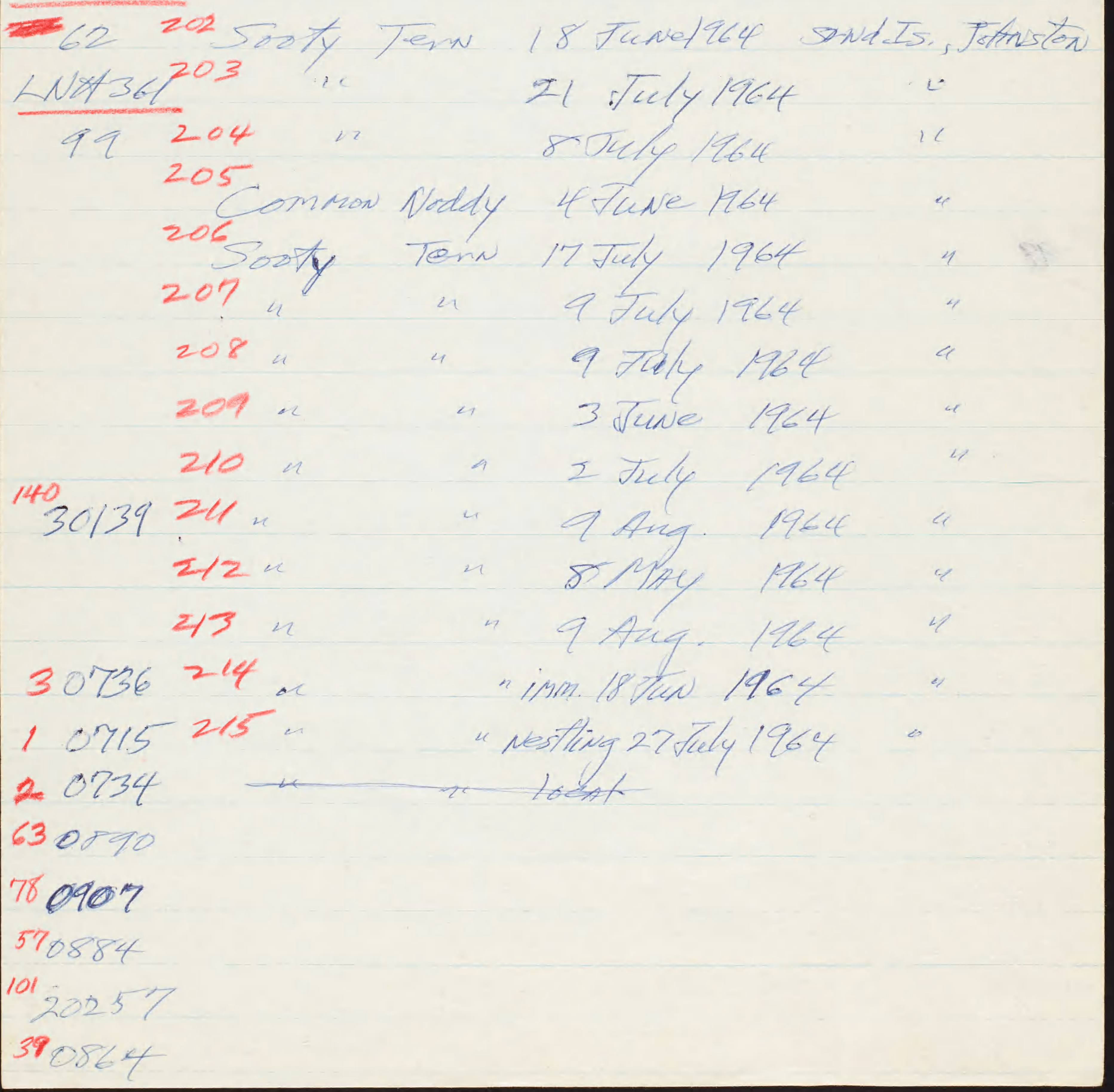




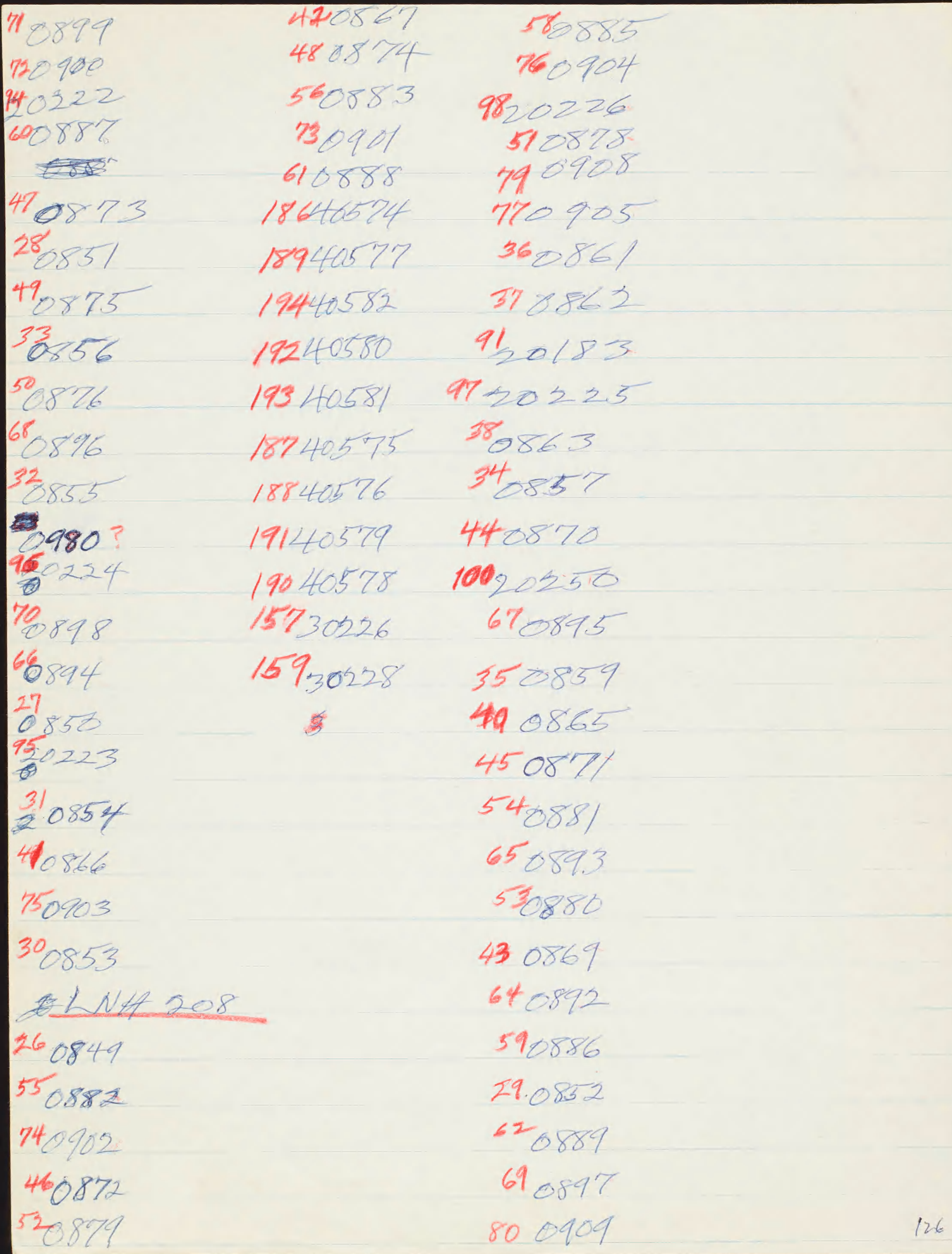




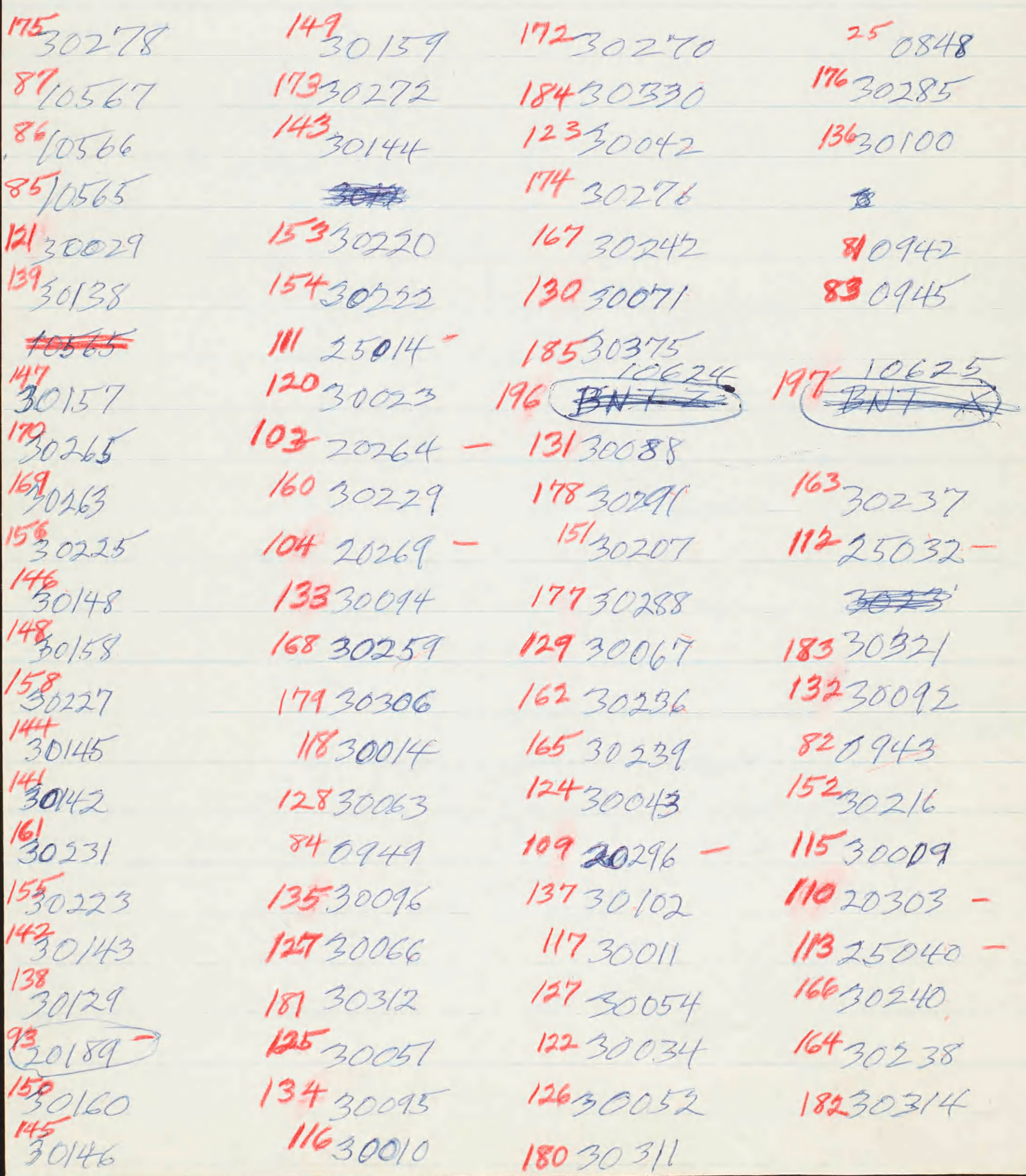




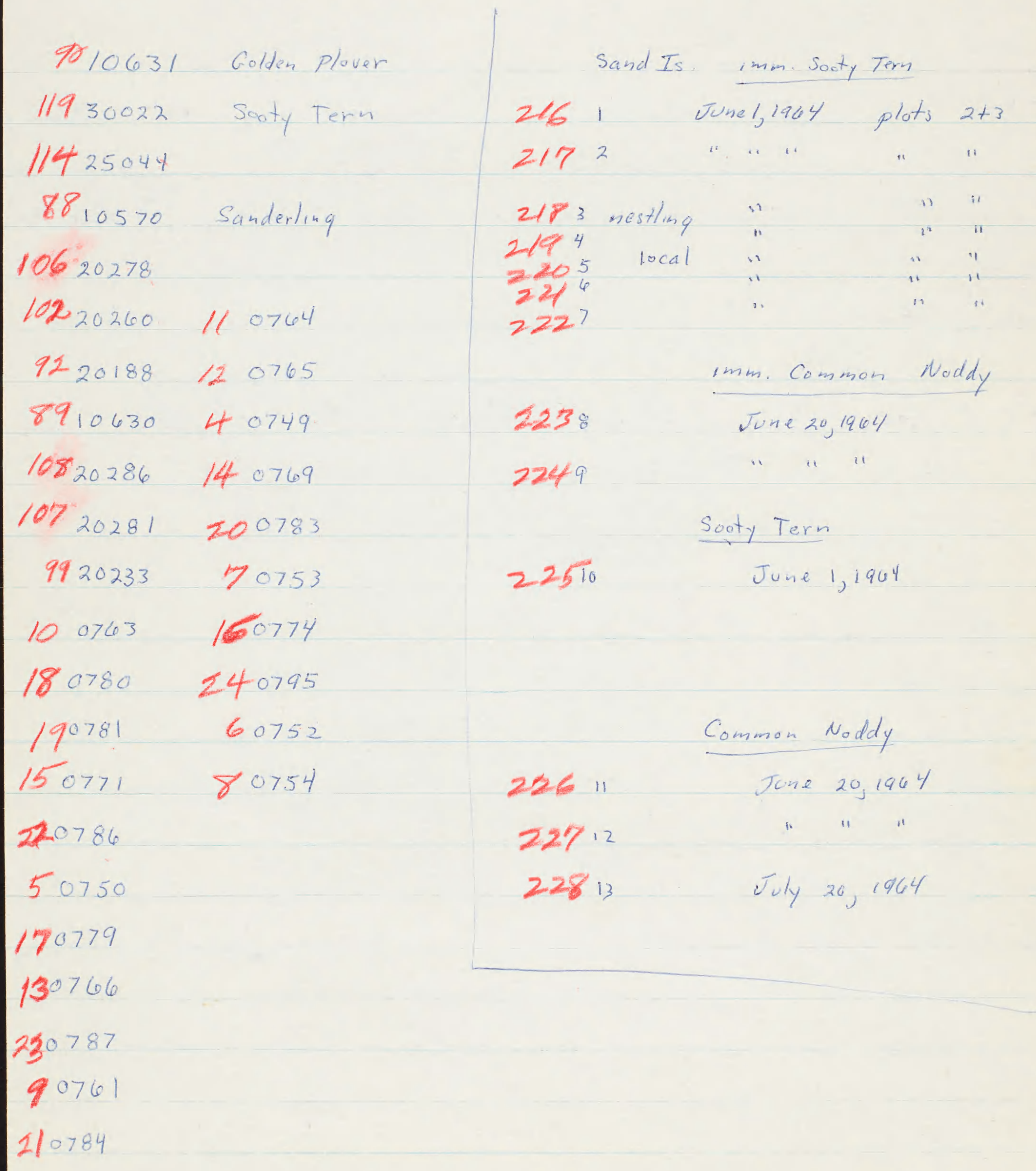

84 
199

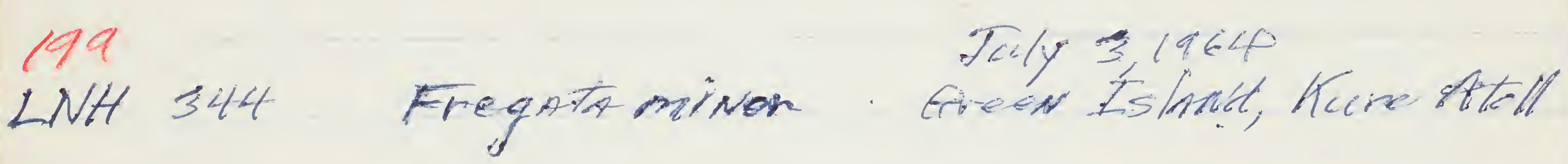

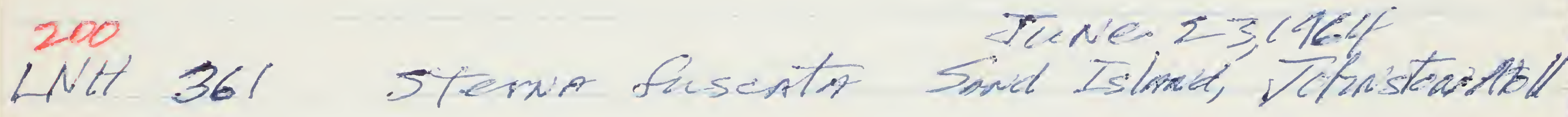

LNA 362

LNA 208

Patadianio phroepygin

Mowi Howaid

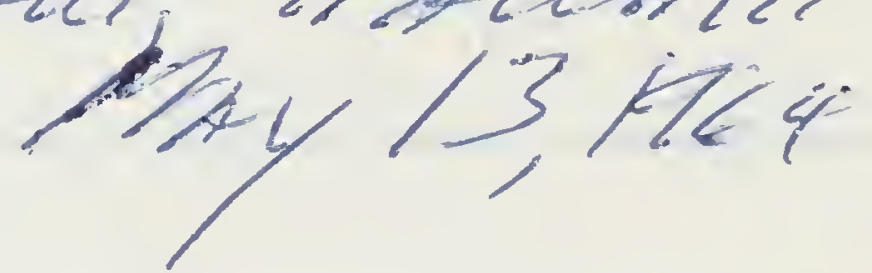


Stomachs

Field

Number

Specles

Date

Iocation

0520 Pterodrome externa

24. May $196511^{\circ} 55^{\prime} \mathrm{N}, 148^{\circ} 00^{\prime} \mathrm{W}$

1051 Eulweria bulweri1

04 May $196520^{\circ} 52^{\prime} \mathbb{N} \cdot 157^{0} 28^{\prime}$,

1052 Sterne fuscata

05 May $196518^{\circ} 33^{\prime} \mathrm{N}, 155^{\circ} 38^{\prime}$ *

1053 Sterocrarlus longicauda

o7 May $196513^{\circ} 20^{\prime} \mathrm{N}, 151^{\circ} 11^{\prime}$ W

1054 Sula dactyletra

08 May $196510^{\circ} 51^{\prime} \mathrm{N}, 149^{\circ} 15^{\prime} \mathrm{W}$

1056 Pterodroma externa

08 May $196510^{\circ} 42^{\prime} \mathrm{N}, 149^{\circ} 13^{\prime} \mathrm{W}$

$1057 \quad$ Sula dectyletra

08 May $196510^{\circ} 43^{\prime} N, 149^{\circ} 13^{\prime}$ W

1058 Pterodroma externa

08 May $196510^{\circ} 37^{\prime} \mathrm{N}, 149^{\circ} 16^{\prime} \mathrm{W}$

1059 Pterodroma externa

$08 \mathrm{May} 196510^{\circ} 35^{\prime} \mathrm{N}, 149^{\circ} 15^{\prime} \mathrm{W}$

1060 Sula dectyletra

$08 \mathrm{Mgy} 196510^{\circ} 36^{\prime} \mathrm{N}, 149^{\circ} 14, \mathrm{~W}$

1061 Pterodroma externa

1062 Puffinus pacificus

1063 Puffinus pacificus

$08 \mathrm{Mey} 196510^{\circ} 35^{\prime} \mathrm{N}, 149^{\circ} 20^{\prime}$ *

08 Mey $196510^{\circ} 33^{\prime} \mathrm{N}, 149^{\circ} 21^{\prime}$ w

08 May $196510^{\circ} 31 \mathrm{~N}, 149^{\circ} 23^{\prime}$ if

1064 Ocenodrome leucorhoe

$08 \mathrm{May}, 196510^{\circ} 24^{4} \mathrm{~N}, 149^{\circ} 29^{\prime} \mathrm{W}$

1065 Pterodroma externa

$08^{\text {May }} 196510^{\circ} 06^{\prime} \mathrm{N}, 149^{\circ} 46^{\prime}$ W

1066 Pterodroma externa

$08 \mathrm{May} 196509^{\circ} 40^{\prime} \mathrm{N}, 150^{\circ} 12^{\prime}$ ต

1068 Sula dactylstra

09 Mey $196508^{\circ} 06^{\prime} \mathrm{N}, 151^{\circ} 47^{\prime}$ w

1069 Sterna fuscata

09 May $196508^{\circ} 01^{\prime}$ N, $152^{\circ} 00^{\prime}$ V

1070 Sterne fusceta

09 Mey $196508^{\circ} \mathrm{O} 1^{\prime} \mathrm{N}, 152^{\circ} \mathrm{OO} \mathrm{\prime}^{\prime} \mathrm{W}$

1071 Puffinus pacificus

09 May $196508^{\circ} 02^{\prime} \mathrm{N}, 152^{\circ} \mathrm{O} 1^{\prime}$ W

1072 Pterodrome externa

$09 \mathrm{May} 196508^{\circ} 02^{\prime} \mathrm{N}, 152^{\circ} 02^{\prime}$ it

1073 Sterna fuscata

09 May $196508^{\circ} 02^{\prime N}, 152^{\circ} 02^{\prime}$ W

1074 Sterna fuscata

$09 \mathrm{May} 196508^{\circ} 03^{\prime} \mathrm{N}, 152^{\circ} 07^{\prime} \mathrm{W}$

1075 Sterna fuscata

09 Mey $196508^{\circ} 03^{\prime}$ N. $15205^{\prime} W$ 
Field.

Numiber

Species

1077 Suls dactylatra

1079 Sula dectylatra

1080 Pterodroma externa

1081 Sula dactylatra

1082 Sterna fuscate

1083 Sula dactyletra

1085 Sula dsctylatra

1086 Pterodrome externa

1087 Stercorarins longicauda

1088 Sule dactylatra

1089 Sterna fuscata

1090 Sterne fuscata

1091 Sule dectyletre

1092 Sterne fuscata

1093 Fuffinus netivitatus

1094 Sule dactylatra

1095 Sula dactyletra

1096 Sula dactyletra

1098 Sula dactylatra

1099 Sula dactylatra

1100 Sule dactyletra

1101 Sterne fuscata

1102 Puffinus pecificus

1103 Sula dect letra
Date

$09 \mathrm{Mey} 1965$

$18 \mathrm{Mey} 1965$

10 May 1965

10 Mey 1965

12 May 1965

11 Mey , 1965

11 May, 1965

11 Mey, 1965

11 May, 1965

11 May 1965

11 May 1965

11. May 1965

11 May 1965

11 May 1965

11 Mey 1965

11 May 1965

11 May 1965

11 May 1965

11 May 1965

11 Mey 1965

11 May 1965

$11 \mathrm{May}, 1965$

1] May 1965

11 May 1965
Location

$0801^{\prime} N, 15245^{\prime} \mathrm{V}$

$0934^{\prime} \mathrm{N}, 15153^{\prime}$ 웡

$0935^{\prime} \mathrm{N}, 15157^{\prime}$ ख

$0935^{\prime} \mathrm{N}, 153 \mathrm{O5}^{\prime} \mathrm{W}$

$0805^{\prime} \mathrm{N}, 155^{4} 46^{\prime}$ औ

$0849 \mathrm{~N}, 15546^{\prime} \mathrm{V}$

$0843^{*} \mathrm{~N}, 15550^{\prime} \mathrm{V}$

$0843^{\prime} \mathrm{N}, 15550^{\prime} \mathrm{W}$

$0840^{\prime} n, 15554^{\prime}$,

$0836^{\prime} \mathrm{N}, 15559^{\prime} \mathrm{y}$

$0833^{\prime} \mathbb{N}, \quad 15614^{\prime}$

$0833^{\prime} \mathrm{N}, 15614^{\prime}$,

$0829^{\prime} \mathrm{N}, 15625^{\prime} \mathrm{W}$

$2127^{\prime} \mathrm{N}, 1.5629^{\prime}$ พ

$08181 \mathrm{~N}, 15641^{\prime} \mathrm{W}$

$0816^{\prime} \mathrm{N}, 15643^{\prime} \mathrm{W}$

$0812^{\prime} \mathrm{N}, 15646^{\prime}$ t

$0811^{*} \mathrm{~N}, 15646^{\prime} \mathrm{V}$

$0812^{\prime} \mathrm{N}, 15646^{\prime}$

$0804^{\prime}$ N, $15655^{\prime} \mathrm{W}$

$0803^{\prime} \mathrm{N}, 15707^{\prime} \mathrm{W}$

$0803^{\prime} \mathrm{N}, 157^{\circ}$. $\mathrm{*}$

$0803^{\prime} \mathbb{N}, 157$ 07'

$0803^{\prime}$ N, $15708^{\prime} \mathrm{W}$ 
Field

Number

Species

1104 Sula dactylatra

1106

Sterna fuscata

1107

Sula dactylatra

1109

Sula dectylatra

1110 Puffinus pacificus

1111 Sula dactylatra

1112 Phaethon lepturus

111. Sterna Puscata

1115 Sterna fuscata

1116 Phacthon lepturus

1143 sterna fuscata

1144 Sterna fuscats

1145 , Sterna fuscata

1161 Puffinus pacificus

1162 Puffinus pacificus

1364 Sterna fuscata

1165 Sterna fuscata

1167 Purfinus pacificus

1169 Sterna fuscata

1171 Puffinus pacificus

1172 Puffinus paeificus

1173 Puffinus pacificus

1175 Gygis alba

1182 Pufrinus pacificus

1184 Sterna fiuscata

1186 Gygis alba
Date

11 May 1965

12 May 1965

12 May 1065

12 May 1965

12 May 1965

12 May 1965

14 May 1965

15 May, 1965

16 May 1965

16 May 1965

21 May 1965

21 May 1965

22 May 1965

23 May 1965

23 May 1965

23 May 1965

23 May 1965

23 May 1965

$25 \mathrm{May} 1965$

23 May 1965

23 May 1965

23 May 1965

2.) May 1965

2) May 1965

2) May 1965

23 May 1965

\section{Location}

$0805^{\prime} \mathrm{N}, 15710^{\circ} \mathrm{W}$

$0815^{\prime} \mathrm{N}, 1600^{\prime} \mathrm{W}$

$0815^{\prime} \mathrm{N}, 16004^{\prime} \mathrm{W}$

$0813^{\prime} \mathrm{N}, 16026^{\prime} \mathrm{W}$

$0812^{\prime} \mathrm{N}, 1604^{\prime} \mathrm{W}$

$0812^{\prime} \mathrm{N}, 16059^{\prime} \mathrm{W}$

$1150^{\prime} \mathrm{N}, 16643^{\prime} \mathrm{W}$

$1332^{\prime} \mathrm{N}, 16945^{\circ} \mathrm{W}$

$1319^{\prime} \mathrm{N}, 17111^{\prime} \mathrm{W}$

$1355^{\prime} \mathrm{N}, 170,4^{\prime} \mathrm{W}$

$1902^{\prime} \mathrm{N}, 17011^{\prime} \mathrm{W}$

$1902^{\prime} \mathrm{N}, 17011^{\prime} \mathrm{W}$

$2059^{\circ} \mathrm{N}, 1680^{\prime} 4^{\prime}$

$213, \mathrm{~N}, \quad 16438^{\prime} \mathrm{W}$

$2133^{\prime} \mathrm{N}, 16458{ }^{\prime} \mathrm{W}$

$2133^{\prime} \mathrm{N}, 1643^{\prime} \mathrm{W}$

$2135^{\prime} \mathrm{N}, 16438 \mathrm{\prime}$

$2133^{\prime} \mathrm{N}, 16430^{*} \mathrm{~W}$

$2133^{\circ} \mathrm{N}, 16438^{\prime} \mathrm{W}$

$2133^{\circ} \mathrm{N}, 16438^{\prime} \mathrm{w}$

$2153^{\prime} \mathrm{N}, 16435^{\prime} \mathrm{w}$

$2133^{\circ} \mathrm{N}, 16438^{\prime} \mathrm{W}$

$2133^{\prime} \mathrm{N}, 16+35^{\prime} \mathrm{W}$

$2134^{\prime} \mathrm{N}, 16437^{\prime} \mathrm{W}$

$2135^{\circ} \mathrm{N}, 16435^{\prime} \mathrm{W}$

$2138^{\circ} \mathrm{N}, 16406^{\prime} \mathrm{W}$ 
Field

Number Species

Date

24 May 1965

24 May 1965

24 May 1965

24 May 1965

24 May 1965

24 May 1965

24 May 1965

24 May 1965

24 May 1965

24 May 1965

24 May 1965

$24 \mathrm{May} 1965$

24 May 1965

May 24, 1965

25 May 1965

24 May 1965

24 May 1965

24 Mey 1065

24 May 1965

24 May 2965

03 March 1965

03 March 3965

0द March 1965

06 Aug, 2965
Location

$2142^{\prime} \mathrm{N}, 16150^{\circ} \mathrm{W}$

$2142^{\prime} \mathrm{N}, 16140^{\prime} \mathrm{W}$

$214^{\circ} \mathrm{N}, 16122^{\prime} W$

$214^{\prime} \mathrm{N}, 16122^{\prime} \mathrm{W}$

$2143^{\prime} \mathrm{N}, 16117^{\circ} \mathrm{W}$

$2144^{\prime} \mathrm{N}, 161.13^{\prime} \mathrm{W}$

$214^{\prime} \mathrm{N}, 16111^{\prime} \mathrm{W}$

$2144^{\prime} N, 161$ 11'

$2144^{\circ} \mathrm{N}, 16111^{\prime} \mathrm{W}$

$214^{\prime} N, 161$ 11' W

$2144^{\prime} \mathrm{N}, 161 \mathrm{OI}^{\prime} \mathrm{W}$

$214^{\circ} \mathrm{N}, 16057^{\prime} \mathrm{W}$

$214^{\prime} \mathrm{N}, 1604^{\prime} \mathrm{W}$

$214^{\circ} \mathrm{N}, 1604^{\circ} \mathrm{W}$

$2145^{\prime} \mathrm{N}, 16046 \cdot \mathrm{m}$

$2145^{\prime} \mathrm{N}, 16046^{\prime} \mathrm{H}$

$2145^{\circ} \mathrm{N}, 16046^{\prime} \mathrm{W}$

$214^{\circ} \mathrm{N}, 16046^{\circ} \mathrm{W}$

$2145^{\prime} \mathrm{N}, 16046^{\prime} \mathrm{W}$

$2145^{\prime} \mathrm{N}, 16046^{\prime} \mathrm{W}$

Atatau Atoll, Tokelau Is.

Atatau Atoll, Tokelau Is.

Atafu Atoll, Tokelau Is.

Green Is., Kure Atoll 
Field

Number

20274

20279 Sterna fuscata

20280 Sterna fuscata

20284 Arenaria interpres

20295 Pluvialis dominica

20309 Erolla melanotos

20310 Erolia melanotos

20311 Erolia melanotos

20318 Arenaria interpres

20323 Arenaria interpres

203,1 Pluvialis dominica

20,59 Pterodroma hypoleuca

20363 Sula leucogaster

20364 Anous stolldus

20365 Pterodroma hypoleuca

20366 Limnodromus sp.

20367 Heteroscelus incanum

20369 Erolia melanotos

20570 Erolia melanotos

20372 Circus cyaneus

20373 Fregata minor

20375 Arenaria interpres

20379 Anas acuta

20380 Pluvialis dominica

20381 Sula sula
Date

18 Aug 1964

28 Aug 1964

28 Aug 1965

06 sep 1964

15 Sep 1964

25 Sep 1964

$25 \operatorname{sep} 1964$

25 Sep 1964

30 sep 1964

02 Oet 1964

O9 Oet 1964

24 Oet 1964

30 Oet 1964

31 oct 1964

O2 Nov 1964

30 oet 1964

30 oct 1964

31 Oet 1964

31 oet 1964

31 oct 1964

03 Nov 1964

06 Nov 1964

O7 Nov 1964

$08 \mathrm{Nov}, 1964$

O9 Nov 1964
Location

Green Is., Kure Atoll

Green Is., Kure Atol1

Green Is., Kure AtolI

Green Is., Kure AtolI

Green Is., Kure Atoll

Green Is., Kure Atoll

Green Is., Kure Atoll

Green Is., Kure AtolI

Green Is, Kure Atoll

Green Is., Kure AtolI

Green Is., Kure Atol1

Green Is., Kure AtolI

Green Is., Kure Atol1

Green Is., Kure Atoll

Green Is., Kure Atoll

Sand I., Midway

Sand I., Midway

Sand I., Midway.

Sand I., Midway

Eastern I., Midway

Green Is., Kure Atoll

Green Is., Kure AtolI

Green Is., Kure Atoll

Green I., Kure Atoll

Green I., Kure Atoll 
Field

Number Species

20382 Larus sp.

20383 Mareca penelope

20403 Larus sp.

20405 Rissa tridactyia

20414 Larus sp.

20415 Larus argentatus

20441 Diomedea nizripes

20445 Oceanodroma markhami

20446 Faleo peregrinus

20447 Larus Sp.

20448 Iarus occidentalis

20449 Larus sp.

20450 Larus sp.

20452 Phaethon rubricauda

20453 Anas acuta

20454 Anas acuta

20456 Gygis alba

20461 Larus sp.

25021 Anous stolidus

25029 Sterna fuscata

25034 Anous stolidus

25036 sterna fuscata

25038 Sterna fuscata

25039 Sterna fuscata

30056 Procelsterna cerulea
Date

$10 \mathrm{Nov} 1964$

$10 \mathrm{Nov} 1964$

$24 \operatorname{Dec} 1964$

30 Dec 1964

$17 \operatorname{Jan} 1965$

$20 \operatorname{Jan} 1965$

26 Feb 1965

OI March 1965

07 March 1965

07 Mareh 1964

08 March 1965

09 March 1965

OS March 1965

08 March 1965

17 March 1965

19 March 1965

24. March 1965

01 Anx 1965

20 Aug 1964

11 Aug 1964

18 fug 1964

15 Aug 1964

25 Aur 1964

OI Sep 1,64

17 Oct 1964
Location

Green I., Kure Atoll

Green I., Kure Atoll

Green I., Kure Atoll

Green I., Kure Atoll

Green I., Kure AtolI

Green I., Kure Atoll

Green I., Kure Atoll

Green I., Kure AtolI

Green I., Kure Atoll

Greed I., Kure Atoll

Green I., Kure Atoll

Green I., Kure Atoll

Green I., Kure Atoll

Green I., Kure Atoll

Green I., Kure Atoll

Green I., Kure Atoll

Green I., Kure Atoll

Green I., Kure AtolI

Sreid I., Johnston AtolI

Sand I., Johnston Atoll

Sand I., Johnston Atoll

Sand I., Johnaton A oll

North I., Johnston Atoll

Sand I., Johnston Atoll

Bizar Atoll, Arumenii I. Marshall I. 
Field

Number

30079 Heteroscelus incanum

30084 Sterna fuscata

30099 Arenaria interpres

30103 Axenaria interpres

20111 Sterna sumatrana

30290 Heteroscelus incarum

30292 Stema sumatrana

3029: Sterna sumatrana

30295 Sterna sumatrana

30256 Stema sumetrana

30297 Sterna sumatrana

30313 Heteroscelus incanum

110002 Heteroscelus incanum

110003 Pluvialts dominte

110004 Pluvialis dominica

110005 Pluvialis domínica

110006 Lobipes lobatus

130008 Squatarole squatarola

110005 Squatarola squatarola

110015 Ereunetes maurd
Date

22 Oct 1964

22 oct 1064

24 oct 1964

24 oet 1964

25 oct 1964

I4 Nov 1964

14 Nov 1964

14 Nov 1964

14 Nov 1964

14 Nov 1964

14 Nov 1964

14 Nov 1964

O7 March 1965

of March 1965

of March 1965

07 March 1965

07 March 1965

13 March 1965

15 March 1965

15 March 1965
Location

Taka I., Taka Atoll, Marshall I. Taka I., Taka Atoll, Marshall I. Loj I., Erikub Atol1, MarshalI I. Loj I., Erikub Atoll, Marshall I. Enego I., Erikub Atoll Marshal1 Is.

Kotabu I., Marin Atoll Gilbert Islands

sandbar $1 / 2 \mathrm{mi}$. SE Tukurere I. Makin Atoll, Gilbert Islunds

sandbar $1 / 2 \mathrm{mi}$, SE Tukurere I. Makin Atol1, Gilbert Islands

sandbar I/2 mi. SE Tukuiere I. Makin Atoll, Gilbert Islands sandbar $1 / 2 \mathrm{mi}$. SE Tukurere I. Makin Atoll, Bilbert Islands

sandbar $1 / 2 \mathrm{mit}$. SE Tukurere I. Makin Atoll, Gilbert Islands

Butaritari I., Makin AtolI Gilbert Islanảs

Laysan I., Hawaiian I.

Laysan I., Hawaiian I.

Laysan I., Hawailan I.

Laysan I., Hawaiian I.

Laysan I., Hawailan I.

Lisianski. I., Hawailan I.

Listansiri I., Hawaitan I.

Pearl. anâ Hermes Reef, Hawaisan Islands 
Fielo

Number

110019

110021 Larus sp.
Date

Location

18 Narch 1965 Pearl and Hermes Reef Hawalian Islanis

I2 March 1965 Insianski I., Hawaiten Is. 


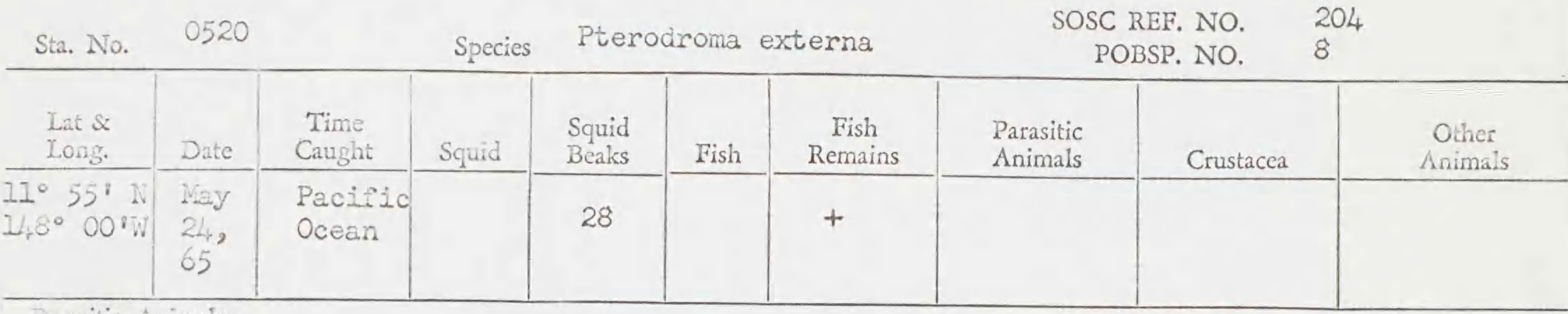

Purasitic Animals:

lots: 2 fish remains

Notes on Sctid: squid beaks

Notes on Fish:

General Notes:

PACIFIC OCEANOGRAPHIC BIOLOGICAL SURVEY PROGRAM SMITHSONIAN OCEANOGRAPHIC SORTING CENTER—SMITHSONIAN INSTITUTION,

Si-USNM-630

S. Willis $1 / 26 / 66$

7.05

\begin{tabular}{|c|c|c|c|c|c|c|c|c|c|}
\hline Sta. No. & 1051 & & Specic & & ia & lwerii & & $\begin{array}{l}\text { No. } \\
\text { No. }\end{array}$ & \\
\hline $\begin{array}{l}\text { Lat \& } \\
\text { Long. }\end{array}$ & Date & $\begin{array}{l}\text { Time } \\
\text { Caught }\end{array}$ & Squid & $\begin{array}{l}\text { Squid } \\
\text { Beaks }\end{array}$ & Fish & $\begin{array}{c}\text { Fish } \\
\text { Remains }\end{array}$ & $\begin{array}{l}\text { Parasitic } \\
\text { Animals }\end{array}$ & Crustacea & $\begin{array}{c}\text { Other } \\
\text { Animals }\end{array}$ \\
\hline $\begin{array}{l}20^{\circ} 52^{\prime} \mathrm{N} \\
157^{\circ} 28^{\prime} \\
\mathrm{H}\end{array}$ & $\begin{array}{l}\text { May } \\
49 \\
65\end{array}$ & $\begin{array}{l}\text { Pacifib } \\
\text { Ocean }\end{array}$ & & & & - & & & \\
\hline
\end{tabular}

Parasitic Animals:

Notes on Squid:

lots: 1 digestedremains

\section{Notes on Fish:}

General Notes:

The remains are beyond identification. 


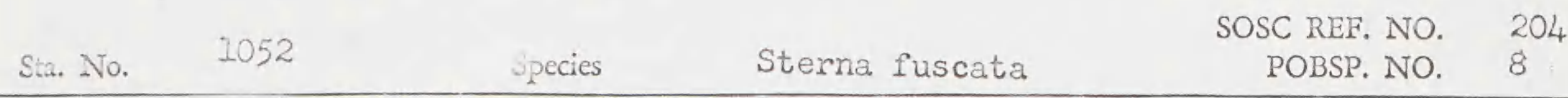

\begin{tabular}{|c|c|c|c|c|c|c|c|c|c|}
\hline $\begin{array}{l}\text { Lat \& } \\
\text { Long. }\end{array}$ & Date & $\begin{array}{l}\text { Time } \\
\text { Caught }\end{array}$ & Squid & $\begin{array}{l}\text { Squid } \\
\text { Beaks }\end{array}$ & Fish & $\begin{array}{l}\text { Fish } \\
\text { Remains }\end{array}$ & $\begin{array}{l}\text { Parasitic } \\
\text { Animals }\end{array}$ & Crustacea & $\begin{array}{l}\text { Other } \\
\text { Animals }\end{array}$ \\
\hline $\begin{array}{l}18^{\circ} 33^{\circ} \mathrm{N} \\
155^{\circ} 38^{\circ} \cdot\end{array}$ & $\begin{array}{l}\text { May } \\
59 \\
65\end{array}$ & $\begin{array}{l}\text { Pacific } \\
\text { Ocean }\end{array}$ & & 3 & & - & & & \\
\hline
\end{tabular}

Parasitic Animals:

lots: 2 squid beaks

Notes on Squid:

digested remains

Notes on Fish:

General Notes:

Digested remains contain several bits of fish scales, squid shell, \& I fish bone.

$7 \cdot 65$$$
-1
$$
PACIFIC OCEANOGRAPHIC BIOLOGICAL SURVEY PROGRAM
SMITHSONIAN OCEANOGRAPHIC SORTING CENTER—SMITHSONLAN WINSTITUIION/66
ST-USNM-3.30

SOSC REF. NO. 204

POBSP. NO. 8

\begin{tabular}{|c|c|c|c|c|c|c|c|c|c|}
\hline $\begin{array}{l}\text { Lat \& } \\
\text { Long. }\end{array}$ & Date & $\begin{array}{l}\text { Time } \\
\text { Caught }\end{array}$ & Squid & $\begin{array}{l}\text { Squid } \\
\text { Beaks }\end{array}$ & Fish & $\begin{array}{c}\text { Fish } \\
\text { Remains }\end{array}$ & $\begin{array}{l}\text { Parasitic } \\
\text { Animals }\end{array}$ & Crustacea & $\begin{array}{l}\text { Other } \\
\text { Animals }\end{array}$ \\
\hline $\begin{array}{l}13^{\circ} 20^{\circ} \mathrm{N} \\
151^{\circ} 11^{\circ}\end{array}$ & $\begin{array}{l}\text { Hay } \\
8, \\
65\end{array}$ & & $\frac{1}{\text { Remains }}$ & & & + & & & \\
\hline
\end{tabular}

Parasitic Animals:

lots: 3 squid

Notes on Squid:

Squid with tentacles detached.

squid remains

fish remains

\section{Notes on Fish:}

General Notes: 
Sta. No. 1054 Species Sula dactylatra

\begin{tabular}{|c|c|c|c|} 
Squid & $\begin{array}{c}\text { Squid } \\
\text { Beaks }\end{array}$ & Fish & $\begin{array}{c}\text { Fish } \\
\text { Remains }\end{array}$ \\
\cline { 1 - 2 } & 19 & & - \\
& & &
\end{tabular}

rasitic Animals:

Noics on Squid:

Notes on Fish:

General Notes:

The remains are beyond identification.

PACIFIC OCEANOGRAPHIC BIOLOGICAL SURVEY PROGRAM

SMITHSONIAN OCEANOGRAPHIC SORTING CENTER—SMITHSONIAN INSTITUTION

BI.UANG.830

7.65

S. Willis $12 / 22 / 65$ lots: 4 cephalopod eyes (5)

debris

squid beaks

digested remains
POBSP. NO. 8

\begin{tabular}{|c|c|c|}
\hline $\begin{array}{l}\text { Parasitic } \\
\text { Animals }\end{array}$ & Crustacea & $\begin{array}{c}\text { Other } \\
\text { Animals }\end{array}$ \\
\hline & & \\
\hline & & \\
\hline
\end{tabular}

ns

Other Animals 


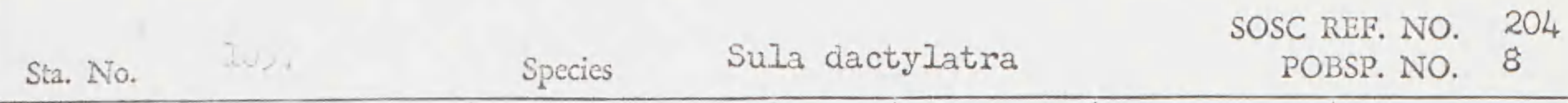

\begin{tabular}{|c|c|c|c|c|c|c|c|c|c|}
\hline $\begin{array}{l}\text { Lat \& } \\
\text { Long. }\end{array}$ & Date & $\begin{array}{l}\text { Time } \\
\text { Camght }\end{array}$ & Squid & $\begin{array}{l}\text { Squid } \\
\text { Beaks }\end{array}$ & Fish & $\begin{array}{c}\text { Fish } \\
\text { Remains }\end{array}$ & $\begin{array}{l}\text { Parasitic } \\
\text { Animals } \\
\end{array}$ & Crustacea & $\begin{array}{c}\text { Other } \\
\text { Animals }\end{array}$ \\
\hline $\begin{array}{l}10^{\circ} 42^{*} \mathrm{~N} \\
149^{\circ} 13 \mathrm{~W}\end{array}$ & $\begin{array}{l}\text { Way } \\
8, \\
65\end{array}$ & $\begin{array}{l}\text { Pacilic } \\
\text { Ocean }\end{array}$ & & & & + & & & \\
\hline
\end{tabular}

Parasitic Animals:

lots: I fish remains

Notes on Squid:

Notes on Fish:

One fish tail is in the remains.

General Notes:

PACIFIC OCEANOGRAPHIC BIOLOGICAL SURVEY PROGRAM

SMITHSONIAN OCEANOGRAPHIC SORTING CENTER—SMITHSONIAN INSTITUTION

51.บรNลล-630

$7 \cdot 68$

S. Wizlis $12 / 14 / 65$

SOSC REF. NO. 204

Sta. No. 1058

Species Pterodroma externa

POBSP. NO. 8

\begin{tabular}{|c|c|c|c|c|c|c|c|c|c|}
\hline $\begin{array}{l}\text { Lat \& } \\
\text { Long. }\end{array}$ & Date & $\begin{array}{l}\text { Time } \\
\text { Caught }\end{array}$ & Squid & $\begin{array}{l}\text { Squid } \\
\text { Beaks }\end{array}$ & Fish & $\begin{array}{c}\text { Fish } \\
\text { Remains }\end{array}$ & $\begin{array}{l}\text { Parasitic } \\
\text { Animals }\end{array}$ & Crustacea & $\begin{array}{l}\text { Other } \\
\text { Animals }\end{array}$ \\
\hline $\begin{array}{l}10^{\circ} 37^{\circ} \mathrm{N} \\
249^{\circ} 26^{\circ} \mathrm{T}\end{array}$ & $\begin{array}{l}\text { May } \\
8, \\
65\end{array}$ & $\begin{array}{l}\text { Pacisic } \\
\text { Ocean }\end{array}$ & & 167 & & - & & & \\
\hline
\end{tabular}

Parasitic Animals:

Iots: 2 squid beaks

Notes on Squid: digested remains

Notes on Fish:

\section{Generail Notes:}

PACIFIC OCEANOGRAPHIC BIOLOGICAL SURVEY PROGRAM SMITHSONIAN OCEANOGRAPHIC SORTING CENTER—SMITHSONIAN INSTITUTION 


\begin{tabular}{|c|c|c|c|c|c|c|c|c|c|}
\hline Sta. No. & & 59 & Specie & Pte & roma & cterna & SOs & $\begin{array}{l}\text { No. } \\
\text { NO. }\end{array}$ & \\
\hline $\begin{array}{l}\text { Lat \& } \\
\text { Long. }\end{array}$ & Date & $\begin{array}{l}\text { Time } \\
\text { Caught }\end{array}$ & Squid & $\begin{array}{l}\text { Squid } \\
\text { Beaks }\end{array}$ & Fish & $\begin{array}{c}\text { Fish } \\
\text { Remains }\end{array}$ & $\begin{array}{l}\text { Parasitic } \\
\text { Animals }\end{array}$ & Crustacea & $\begin{array}{l}\text { Other } \\
\text { Animals }\end{array}$ \\
\hline $\begin{array}{l}35^{\mathrm{I}} \mathrm{N} \\
9^{\circ} 15^{\mathrm{I} W}\end{array}$ & $\begin{array}{l}\text { May } \\
8, \\
65\end{array}$ & $\begin{array}{l}\text { Pacific } \\
\text { Ocean }\end{array}$ & & 23 & & + & $\begin{array}{c}\text { Nematode } \\
\text { (I) }\end{array}$ & & \\
\hline
\end{tabular}

Parasitic Animals:

lots: 3 squid beaks

Notes on Squid: fish remains nematode

Notes on Fish:

General Notes:

PACIFIC OCEANOGRAPHIC BIOLOGICAL SURVEY PROGRAM

SMITHISONIAN OCEANOGRAPHIC SORTING CENTER_SMITHSONIAN INSTITUTION

S. Willis $12 / 8 / 65$

5i-บBNAM-830

$7 \cdot 65$

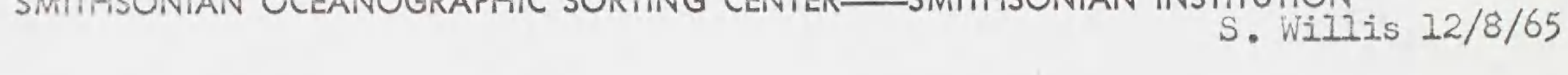

\begin{tabular}{|c|c|c|c|c|c|c|c|c|c|}
\hline Sta. No. & 10 & & Specie & Sul & lacty & ra & & $\begin{array}{l}\text { No. } \\
\text { No. }\end{array}$ & \\
\hline $\begin{array}{l}\text { Lat \& } \\
\text { Long. }\end{array}$ & Date & $\begin{array}{l}\text { Time } \\
\text { Caught }\end{array}$ & Squid & $\begin{array}{l}\text { Squid } \\
\text { Beaks }\end{array}$ & Fish & $\begin{array}{c}\text { Fish } \\
\text { Remains }\end{array}$ & $\begin{array}{l}\text { Parasitic } \\
\text { Animals }\end{array}$ & Crustacea & $\begin{array}{l}\text { Other } \\
\text { Animals }\end{array}$ \\
\hline $\begin{array}{r}36^{\circ} \mathrm{N} \\
-20: \mathrm{N}\end{array}$ & $\begin{array}{l}\text { Nay } \\
8\end{array}$ & $\begin{array}{l}\text { Pacilic } \\
\text { Ocean }\end{array}$ & & 1 & & + & & & \\
\hline
\end{tabular}

Parasitic Animals:

lots: 2 squid bosks

Notes on Squid: fish remains

Notes on Fish:

Fish remains contain one fish tail.

General Notes: 
SOSC REF. NO. 204

Sta. No. 1061

Species

Pterodroma externa POBSP. NO.

8

\begin{tabular}{|c|c|c|c|c|c|c|c|c|c|}
\hline $\begin{array}{l}\text { Lat \& } \\
\text { Long. }\end{array}$ & Date & $\begin{array}{l}\text { Time } \\
\text { Caught }\end{array}$ & Squid & $\begin{array}{l}\text { Squid } \\
\text { Beaks }\end{array}$ & Fish & $\begin{array}{c}\text { Fish } \\
\text { Remains }\end{array}$ & $\begin{array}{l}\text { Parasitic } \\
\text { Animals }\end{array}$ & Crustacea & $\begin{array}{l}\text { Other } \\
\text { Animals }\end{array}$ \\
\hline $\begin{array}{r}10^{\circ} 35^{\circ} \mathrm{N} \\
149^{\circ} 20^{\circ} \mathrm{W}\end{array}$ & $\begin{array}{l}\text { May } \\
89 \\
65\end{array}$ & $\begin{array}{l}\text { Pacific } \\
\text { Ocean }\end{array}$ & & 304 & & - & & & \\
\hline
\end{tabular}

Parasitic Animals:

lots: 2 squid beaks

Notes on Squid:

digested remains

Notes on Fish:

General Notes:

Several squid beak fragments and a few fish bones were left in the digested remains.

PACIFIC OCEANOGRAPHIC BIOLOGICAL SURVEY PROGRAM

SMITHSONIAN OCEANOGRAPHIC SORTING CENTER—SMITHSONIAN INSTITUTION WIIIS 3/2/66

8i-บรกลก-830

7.05

\begin{tabular}{|c|c|c|c|c|c|c|c|c|c|}
\hline Sta. No. & 20 & & Specie: & Puff & $2 \mathrm{pa}$ & icus & & $\begin{array}{l}\text { NO. } 20 \\
\text { NO. } 8\end{array}$ & \\
\hline $\begin{array}{l}\text { Lat \& } \\
\text { Long. }\end{array}$ & Date & $\begin{array}{c}\text { Time } \\
\text { Caught }\end{array}$ & Squid & $\begin{array}{l}\text { Squid } \\
\text { Beaks } \\
\end{array}$ & Fish & $\begin{array}{c}\text { Fish } \\
\text { Remains }\end{array}$ & $\begin{array}{l}\text { Parasitic } \\
\text { Animals } \\
\end{array}$ & Crustacea & $\begin{array}{c}\text { Other } \\
\text { Animals }\end{array}$ \\
\hline $\begin{array}{l}10^{\circ} 33^{\circ} \mathrm{N} \\
149^{\circ} 21^{\circ} \mathrm{W}\end{array}$ & $\begin{array}{l}\text { May } \\
8 ; \\
65\end{array}$ & $\begin{array}{l}\text { Pacific } \\
\text { Ocean }\end{array}$ & & & & - & & & \\
\hline
\end{tabular}

Notes on Squid:

Iots: I digested remains

Notes on Fish:

General Notes:

One squid beak was left in the remains. 


$\begin{array}{llr}\text { Sta. No. } 1063 \text { Species Puffinus pacificus } & \text { SOSC REF. NO. }{ }^{204} \\ \text { POBSP. NO. } & 8\end{array}$

\begin{tabular}{|c|c|c|c|c|c|c|c|c|c|}
\hline $\begin{array}{l}\text { Lat \&t } \\
\text { Long. }\end{array}$ & Date & $\begin{array}{l}\text { Time } \\
\text { Caught }\end{array}$ & Squid & $\begin{array}{l}\text { Squid } \\
\text { Beaks } \\
\end{array}$ & Fish & $\begin{array}{c}\text { Fish } \\
\text { Remains }\end{array}$ & $\begin{array}{l}\text { Parasitic } \\
\text { Animals }\end{array}$ & Crustacea & $\begin{array}{l}\text { Other } \\
\text { Animals }\end{array}$ \\
\hline $\begin{array}{l}20^{\circ} 31 \cdot \mathrm{N} \\
149^{\circ} \\
23^{\circ} \mathrm{W}\end{array}$ & $\begin{array}{l}\text { Hay } \\
8 \\
65\end{array}$ & $\begin{array}{l}\text { Pacific } \\
\text { Ocean }\end{array}$ & & & & - & Nematodes (2) & & \\
\hline
\end{tabular}

Parasitic Animals:

lots: 2 nematodes

Notes on Squid:

aigested remains

Notes on Fish:

General Notes:

The digested remains are beyond identification.

PACIFIC OCEANOGRAPHIC BIOLOGICAL SURVEY PROGRAM

SMITHSONIAN OCEANOGRAPHIC SORTING CENTER—SMITHSONIAN INSTITUTION

Si-USNM-630

S. Willis $2 / 21 / 66$

$7 \cdot 65$

SOSC REF. NO. 204

Sta. No. 2064

Species Oceanodroma Ieucorhoa

POBSP. NO. 8

\begin{tabular}{|c|c|c|c|c|c|c|c|c|c|}
\hline $\begin{array}{l}\text { Lat \& } \\
\text { Long. }\end{array}$ & Date & $\begin{array}{l}\text { Time } \\
\text { Caught }\end{array}$ & Squid & $\begin{array}{l}\text { Squid } \\
\text { Beaks }\end{array}$ & Fish & $\begin{array}{c}\text { Fish } \\
\text { Remains }\end{array}$ & $\begin{array}{l}\text { Parasitic } \\
\text { Animals }\end{array}$ & Crustacea & $\begin{array}{l}\text { Other } \\
\text { Animals }\end{array}$ \\
\hline $\begin{array}{l}10^{\circ} 24^{\circ} \mathrm{N} \\
149^{\circ} 29^{\circ} \mathrm{W}\end{array}$ & $\begin{array}{l}\text { May } \\
8, \\
65\end{array}$ & $\begin{array}{l}\text { Pacific } \\
\text { Ocean }\end{array}$ & & & & - & & & \\
\hline
\end{tabular}

Parasitic Animals:

lots: 1 debris

Notes on Squid:

Notes on Fish:

General Notes:

One (I) squid beak was left in the debris. 
SOSC REF. NO. 204

\begin{tabular}{c|c} 
Sta. No. & 1065 \\
\hline Lat \& & \\
Long. & Date \\
\hline $10^{\circ} 06^{\prime} \mathrm{N}$ & Nay \\
$149^{\circ} 46^{\circ}$ & 8, \\
& 65 \\
\hline
\end{tabular}

Species Pterodroma externa

$\mid$\begin{tabular}{|l|}
$\begin{array}{c}\text { Time } \\
\text { Caught }\end{array}$ \\
\hline $\begin{array}{l}\text { Pacific } \\
\text { Ocean }\end{array} \mid$
\end{tabular}

Parasitic Animals:

Notes on Squid:

lots: I fish remains

\section{Notes on Fish:}

\section{General Notes:}

\section{PACIFIC OCEANOGRAPHIC BIOLOGICAL SURVEY PROGRAM}

SMITHSONIAN OCEANOGRAPHIC SORTING CENTER—SMITHSONIAN INSTITUTION 


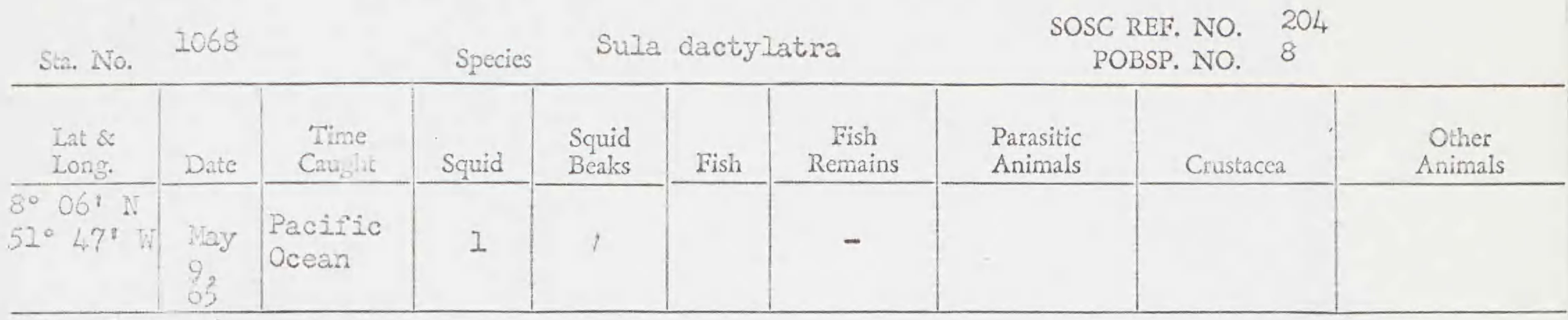

Parasitic Animals:

lots: 2 digested remains

Notes on Squid:

Squid without tentacles. squid

Notes on Fish:

General Notes:

One (1) squid beak was left in the remains.

PACIFIC OCEANOGRAPHIC BIOLOGICAL SURVEY PROGRAM

SMITHSONIAN OCEANOGRAPHIC SORTING CENTER—SMITHSONIAN INSTITUTION

Si-Usพm-630

$7 \cdot 65$

S. Willis $12 / 22 / 65$

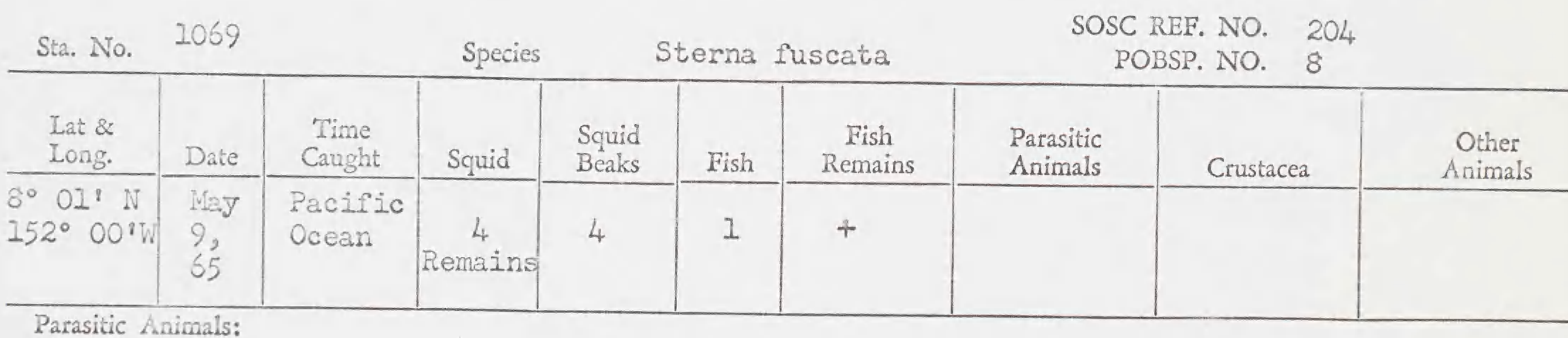

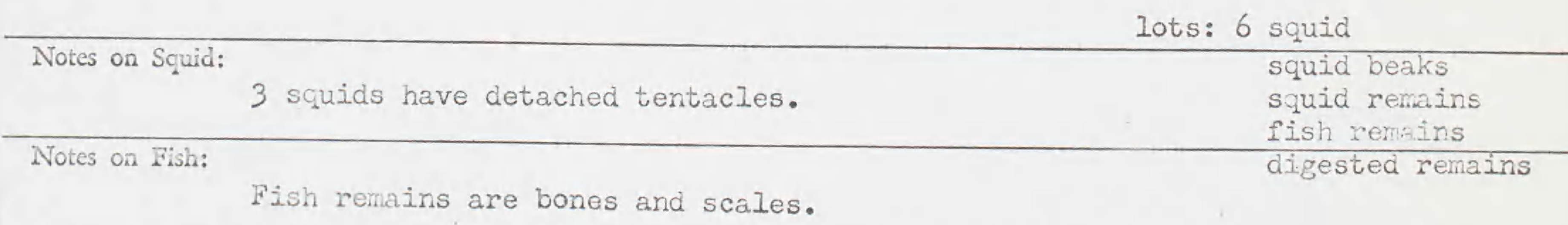

General Notes: 


\begin{tabular}{|c|c|c|c|c|c|c|c|c|c|}
\hline Sta. No. & 1070 & & Species & & $2 a f^{2}$ & ata & & $\begin{array}{l}\text { NO. } \\
\text { NO. }\end{array}$ & \\
\hline $\begin{array}{l}\text { Lat \& } \\
\text { Long. }\end{array}$ & Date & $\begin{array}{l}\text { Time } \\
\text { Caught }\end{array}$ & Squid & $\begin{array}{l}\text { Squid } \\
\text { Beaks }\end{array}$ & Fish & $\begin{array}{c}\text { Fish } \\
\text { Remains } \\
\end{array}$ & $\begin{array}{l}\text { Parasitic } \\
\text { Animals }\end{array}$ & Crustacea & $\begin{array}{l}\text { Other } \\
\text { Animals }\end{array}$ \\
\hline $\begin{array}{l}8^{\circ} 02^{\circ} \mathrm{N} \\
152^{\circ} 00^{\circ} \\
\mathrm{W}\end{array}$ & $\begin{array}{l}\text { May } \\
9 \\
65\end{array}$ & $\begin{array}{l}\text { Pacific } \\
\text { Ocean }\end{array}$ & $\begin{array}{c}3 \\
\text { Remains }\end{array}$ & & & - & & & \\
\hline
\end{tabular}

Notes on Squid:

Iots: 4 squid

one squid had detached tentacles. squid remains digested renains debris

Notes on Fish:

General Notes: 1. One squid beaks was left in the digested remains.

2. Bits of wood particles comprise the debris.

PACIFIC OCEANOGRAPHIC BIOLOGICAL SURVEY PROGRAM

SMITHSONIAN OCEANOGRAPHIC SORTING CENTER—SMITHSONIAN INSTITUTION

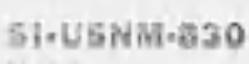
S. Willis $2 / 28 / 66$

7.68

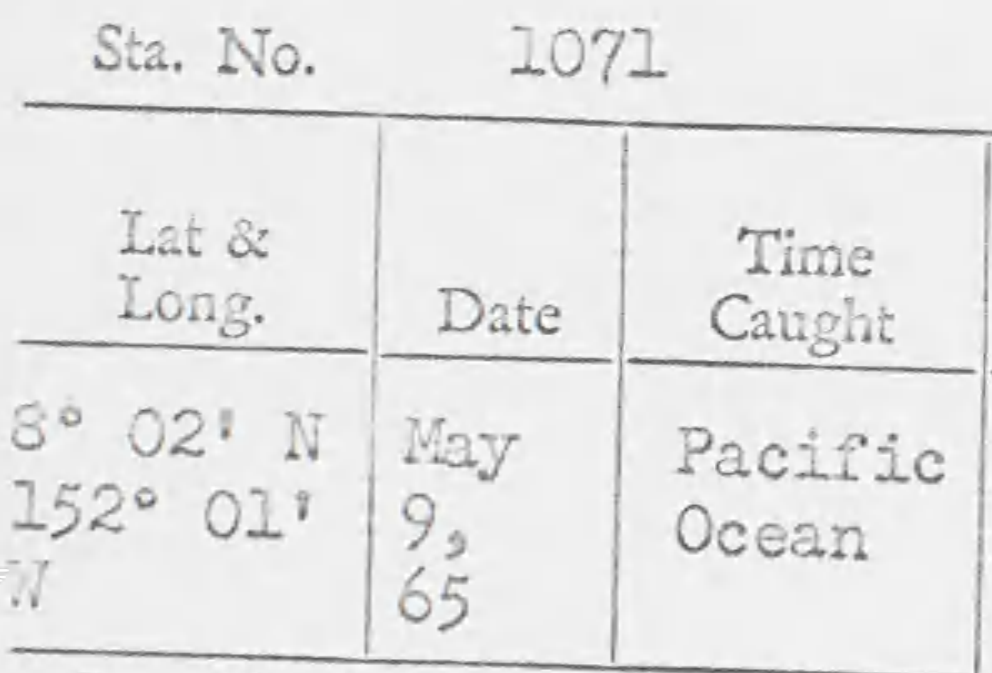

Parasitic Animals:

\begin{tabular}{|c|c|c|} 
Species & Puffinus pa \\
Squid & $\begin{array}{c}\text { Squid } \\
\text { Beaks }\end{array}$ & Fish \\
\hline 8 & & \\
\hline
\end{tabular}

pacificus

\begin{tabular}{c} 
Fish \\
Remains \\
\hline-
\end{tabular}

SOSC REF. NO. 204

POBSP. NO. 8

\begin{tabular}{|c|c|c}
$\begin{array}{c}\text { Parasitic } \\
\text { Animals }\end{array}$ & Crustacea & $\begin{array}{c}\text { Other } \\
\text { Animals }\end{array}$ \\
\hline & &
\end{tabular}

Notes on Squid:

Iots: 2 squid

Squid have tentacles detached.

digested remains

Notes on Fish:

General Notes:

The digested remains are beyond identification. 
SOSC REF. NO. 204

Sta. No. $1072 \quad$ Species Fterodroma externa

POBSP. NO. 8

\begin{tabular}{|c|c|c|c|c|c|c|c|c|c|}
\hline $\begin{array}{l}\text { Lat \& } \\
\text { Long. }\end{array}$ & Date & $\begin{array}{l}\text { Time } \\
\text { Caught }\end{array}$ & Squid & $\begin{array}{l}\text { Squid } \\
\text { Beaks }\end{array}$ & Fish & $\begin{array}{l}\text { Fish } \\
\text { Remains }\end{array}$ & $\begin{array}{l}\text { Parasitic } \\
\text { Animals }\end{array}$ & Crustacea & $\begin{array}{l}\text { Other } \\
\text { Animals }\end{array}$ \\
\hline $\begin{array}{lll}8^{\circ} & 02^{\circ} & N \\
152^{\circ} & 02^{\prime}\end{array}$ & $\begin{array}{l}\text { Xay } \\
9 . \\
65\end{array}$ & $\begin{array}{l}\text { Pacific } \\
\text { Ocean }\end{array}$ & & 22 & & - & & & \\
\hline
\end{tabular}

Parasitic Ánimals:

lots: 2 squid beaks

Notes on Squid:

digested remains

Notes on Fish:

General Notes:

The remains are beyond identification.

PACIFIC OCEANOGRAPHIC BIOLOGICAL SURVEY PROGRAM

SMITHSONIAN OCEANOGRAPHIC SORTING CENTER—SMITHSONIAN INSTITUTION

Si-USNA4-630

S. Willis $12 / 8 / 65$

SOSC REF. NO. 204

Sta. No. 2073

Species

Sterna fuscata

POBSP. NO. 8

\begin{tabular}{|c|c|c|c|c|c|c|c|c|c|}
\hline $\begin{array}{l}\text { Lat \& } \\
\text { Long. }\end{array}$ & Date & $\begin{array}{l}\text { Time } \\
\text { Caught }\end{array}$ & Squid & $\begin{array}{l}\text { Squid } \\
\text { Beaks }\end{array}$ & Fish & $\begin{array}{c}\text { Fish } \\
\text { Remains }\end{array}$ & $\begin{array}{l}\text { Parasitic } \\
\text { Animals }\end{array}$ & Crustacea & $\begin{array}{l}\text { Other } \\
\text { Animals }\end{array}$ \\
\hline $\begin{array}{l}8^{\circ} 02^{\circ} \mathrm{N} \\
252^{\circ} 02^{\circ} \mathrm{W}^{\circ}\end{array}$ & $\begin{array}{l}\text { May } \\
92 \\
65\end{array}$ & $\begin{array}{l}\text { Pacific } \\
\text { Ocean }\end{array}$ & 4 & & & - & & & \\
\hline
\end{tabular}

Parasitic Animals:

lots: 2 squids

Notes on Squid: one squid with tentacles; other with tentacles detached. digested remains

Notes on Fish:

General Notes:

identipicateral wood particles are in the digested remains; other contents are beyond

PACIFIC OCEANOGRAPHIC BIOLOGICAL SURVEY PROGRAM

SMITHSONIAN OCEANOGRAPHIC SORTING CENTER—SMITHSONIAN INSTITUTION

S. WiIIis $1 / 4 / 66$ 
Sta. No. 1074

\begin{tabular}{|c|c|c|c|c|c|c|}
\hline $\begin{array}{l}\text { Lat \& } \\
\text { Long. }\end{array}$ & Dute & $\begin{array}{l}\text { Time } \\
\text { Caught }\end{array}$ & Squid & $\begin{array}{l}\text { Squid } \\
\text { Beaks }\end{array}$ & Fish & $\begin{array}{c}\text { Fish } \\
\text { Remains }\end{array}$ \\
\hline $\begin{array}{l}8^{\circ} 03^{\circ} \mathrm{N} \\
152^{\circ} 07^{\circ} \\
W\end{array}$ & $\begin{array}{l}\text { Nay } \\
99 \\
65\end{array}$ & $\begin{array}{l}\text { Pacific } \\
\text { Ocean }\end{array}$ & 2 & & 2 & + \\
\hline
\end{tabular}

Parasitic Animals:
SOSC REF. NO. 204 POBSP. NO. 8

Notes on Squid:

The squid have detached tentacles.

lots: 3 squids

fish

fish remains

\begin{tabular}{|l|l|c}
$\begin{array}{c}\text { Parasitic } \\
\text { Animals }\end{array}$ & Crustacea & $\begin{array}{c}\text { Other } \\
\text { Animals }\end{array}$ \\
\hline & & \\
& &
\end{tabular}

Notes on Fish:

1. The fish's heads are digested.

2. The fish remains are mostly bones. (also scales)

General Notes:

PACIFIC OCEANOGRAPHIC BIOLOGICAL SURVEY PROGRAM

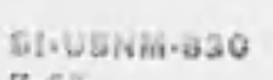

$7 \cdot 65$
SMITHSONIAN OCEANOGRAPHIC SORTING CENTER—SMITHSONIAN INSTITUTION

S. Willis $2 / 28 / 66$

SOSC REF. NO. 204

Sta. No. $1075 \quad$ Species Sterna fuscata

POBSP. NO. 8

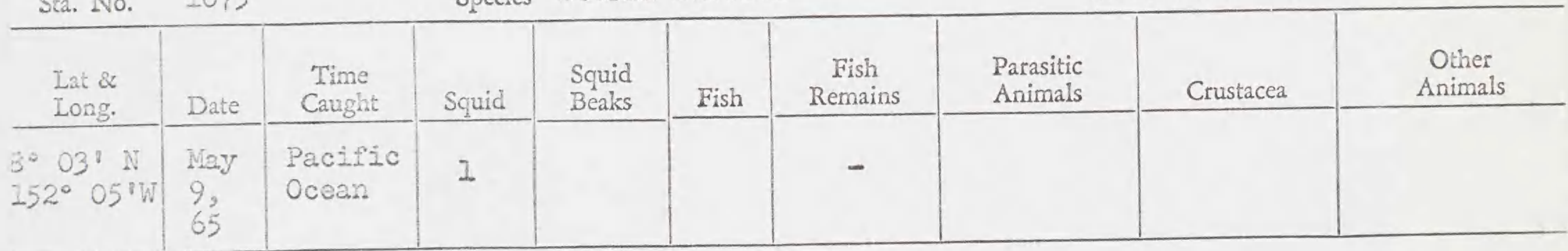

Parasitic Animais:

lots: 2 squid

Notes on Squid: Squid has tentacles attached. digested remains

Notes on Fish:

General Notes:

The remains are beyond identification.

PACIFIC OCEANOGRAPHIC BIOLOGICAL SURVEY PROGRAM SMITHSONIAN OCEANOGRAPHIC SORTING CENTER—SMITHSONIAN INSTITUTION 
Sta, No. $\quad 2077$

Species

Sula dactylatra

\begin{tabular}{|c|c|c|c|c|c|c|c|c|c|}
\hline $\begin{array}{l}\text { Lat \& } \\
\text { Long. }\end{array}$ & Date & $\begin{array}{c}\text { Time } \\
\text { Caught }\end{array}$ & Squid & $\begin{array}{l}\text { Squid } \\
\text { Beaks }\end{array}$ & Fish & $\begin{array}{c}\text { Fish } \\
\text { Remains }\end{array}$ & $\begin{array}{l}\text { Parasitic } \\
\text { Animals }\end{array}$ & Crustacea & $\begin{array}{l}\text { Other } \\
\text { Animals }\end{array}$ \\
\hline $\begin{array}{l}8^{\circ} 01^{\prime} \mathrm{N} \\
252^{\circ} 45^{\circ} \mathrm{W}\end{array}$ & $\begin{array}{l}\text { May } \\
99 \\
65\end{array}$ & $\begin{array}{l}\text { Pacilic } \\
\text { Ocean }\end{array}$ & & 7 & & + & & & \\
\hline
\end{tabular}

Parasitic Animals:

lots: 2 squid beaks

Notes on Squid:

Notes on Fish:

General Notes:

PACIFIC OCEANOGRAPHIC BIOLOGICAL SURVEY PROGRAM

SMITHSONIAN OCEANOGRAPHIC SORTING CENTER—SMITHSONIAN INSTITUTION

S. Willis $1 / 26 / 66$

B1-UENM-ख30

7.65

\begin{tabular}{|c|c|c|c|c|c|c|}
\hline Sta. No. & 1079 & & Specic & Sula & atyl & \\
\hline $\begin{array}{l}\text { Lat \& } \\
\text { Long. }\end{array}$ & Date & $\begin{array}{l}\text { Time } \\
\text { Caught }\end{array}$ & Squid & $\begin{array}{l}\text { Squid } \\
\text { Beaks } \\
\end{array}$ & Fish & $\begin{array}{c}\text { Fish } \\
\text { Remains }\end{array}$ \\
\hline $\begin{array}{l}34^{\circ} \mathrm{N} \\
51^{\circ} 53^{\circ}\end{array}$ & $\begin{array}{l}\text { May } \\
\frac{28}{65}\end{array}$ & $\begin{array}{l}\text { Pacipic } \\
\text { Ocean }\end{array}$ & & 25 & & + \\
\hline
\end{tabular}

SOSC REF. NO.

204

POBSP. NO.

\begin{tabular}{|c|c|c|c|c|} 
Parasitic \\
Animals \\
\hline
\end{tabular}

\begin{tabular}{|c|c|} 
Crustacea & $\begin{array}{c}\text { Other } \\
\text { Animals }\end{array}$ \\
\hline $\begin{array}{c}\text { Isopods (2) } \\
\text { Isopod - } \\
\text { remains }\end{array}$ & \\
\hline
\end{tabular}

Parasitic Animals:

lots: 5 squid beaks cephalopod eyes 6 isopods isopod remains

Notes on Squid: fish remains

Notes on Fish:

General Notes:

The isopod remains consist of logs and bits of carapace.

PACIFIC OCEANOGRAPHIC BIOLOGICAL SURVEY PROGRAM

SMITHSONIAN OCEANOGRAPHIC SORTING CENTER—SMITHSONIAN INSTITUTION 
SOSC REF. NO. 204

Sta. No. 1080

Species Pterodroma externa.

POBSP. NO. 8

\begin{tabular}{|c|c|c|c|c|c|c|c|c|c|}
\hline $\begin{array}{l}\text { Lat \& } \\
\text { Long. }\end{array}$ & Date & $\begin{array}{l}\text { Time } \\
\text { Caught }\end{array}$ & Squid & $\begin{array}{l}\text { Squid } \\
\text { Beaks }\end{array}$ & Fish & $\begin{array}{c}\text { Fish } \\
\text { Remains }\end{array}$ & $\begin{array}{l}\text { Parasitic } \\
\text { Animals }\end{array}$ & Crustacea & $\begin{array}{l}\text { Other } \\
\text { Animals }\end{array}$ \\
\hline $\begin{array}{l}9^{\circ} 35^{\circ} \mathrm{N} \\
152^{\circ} 07^{\circ} \\
W\end{array}$ & $\begin{array}{l}\text { Nay } \\
\frac{10}{65}\end{array}$ & $\begin{array}{l}\text { Pacific } \\
\text { Ocean }\end{array}$ & & & & - & & & \\
\hline
\end{tabular}

Parasitic Animals:

lots: I cephalopod remains

Notes on Squid:

Notes on Fish:

General Notes:

The cephalopod remains consist of a $1 / 2$ squid and remains of an octopus.

PACIFIC OCEANOGRAPHIC BIOLOGICAL SURVEY PROGRAM

SMITHSONIAN OCEANOGRAPHIC SORTING CENTER—SMITHSONIAN INSTITUTION

รก-บธผพิัยวอ

7.05

S. Willis $2 / 2 / 66$

\begin{tabular}{|c|c|c|c|c|c|c|c|c|c|}
\hline Sta. No. & 1081 & & Species & Sula & acty] & & & $\begin{array}{l}\text { NO. } 20 \\
\text { NO. } 8\end{array}$ & \\
\hline $\begin{array}{l}\text { Lat \& } \\
\text { Long. }\end{array}$ & Date & $\begin{array}{c}\text { Time } \\
\text { Caught }\end{array}$ & Squid & $\begin{array}{l}\text { Squid } \\
\text { Beaks } \\
\end{array}$ & Fish & $\begin{array}{c}\text { Fish } \\
\text { Remains } \\
\end{array}$ & $\begin{array}{l}\text { Parasitic } \\
\text { Animals } \\
\end{array}$ & Crustacea & $\begin{array}{l}\text { Other } \\
\text { Animals }\end{array}$ \\
\hline $\begin{array}{l}9^{\circ} 35^{\prime} \mathrm{N} \\
153^{\circ} 05^{\prime} \\
7\end{array}$ & $\begin{array}{l}\text { May } \\
20, \\
65\end{array}$ & $\begin{array}{l}\text { Paciric } \\
\text { Ocean }\end{array}$ & & & 1 & + & & & \\
\hline
\end{tabular}

lots: 2 fish

Notes on Squid:

fish remains

Notes on Fish:

The $x$ in is digested.

General Notes: 


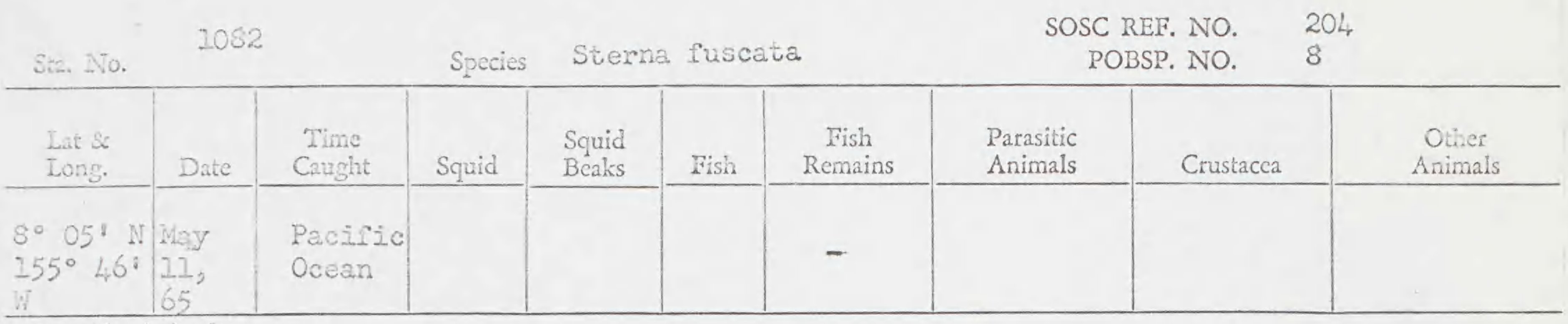

Parasitic Arimals:

lots: 1 digested remains

Notes on Squid:

Notes on Fish:

General Notes:

The remains are beyond identificatione

PACIFIC OCEANOGRAPHIC BIOLOGICAL SURVEY PROGRAM

SMITHISONIAN OCEANOGRAPHIC SORTING CENTER—SMITHSONIAN INSTITUTION

SH.UลN:-630

$7 \cdot 65$

S. Willis $12 / 8 / 65$

Sta. No. 2083

\begin{tabular}{|c|c|}
\hline $\begin{array}{l}\text { Lat \& } \\
\text { Long. }\end{array}$ & Date \\
\hline $\begin{array}{l}8^{\circ} 49^{\circ} \\
155^{\circ} 46^{\circ} \\
\text { T. }\end{array}$ & $\begin{array}{l}\text { May } \\
\frac{21}{65}\end{array}$ \\
\hline
\end{tabular}

Parasitic Animals:

Notes on Squid:

Squid with tentacles detached.

lots: 7 squids

\begin{tabular}{|c|c|c|c|c|}
$\begin{array}{c}\text { Time } \\
\text { Caught }\end{array}$ & Squid & $\begin{array}{c}\text { Squid } \\
\text { Beaks }\end{array}$ & Fish & $\begin{array}{c}\text { Fish } \\
\text { Remains }\end{array}$ \\
\hline $\begin{array}{lll}\text { Pacisic } \\
\text { Ocean }\end{array}$ & $\begin{array}{c}3 \\
\text { Remaing }\end{array}$ & 34 & 1 & + \\
& & 1 & \\
\hline
\end{tabular}

Species Sules dactylatra.

SOSC REF. NO. 204 POBSP. NO.

\begin{tabular}{c|c|c}
$\begin{array}{c}\text { Parasitic } \\
\text { Animals }\end{array}$ & Crustacea & $\begin{array}{c}\text { Other } \\
\text { Animals }\end{array}$ \\
\hline & Isopods (2) & \\
\end{tabular}
sपuid beaks fish fish remains

Notes on Fish:

Fish with head digested, body partially digested. isopods digested remains squid remains

General Notes:

One isopod has head detached. 
Sta. No. 1085 Species

|

\begin{tabular}{|c|c|c|}
\hline $\begin{array}{l}\text { Lat \& } \\
\text { Long. }\end{array}$ & Date & $\begin{array}{l}\text { Time } \\
\text { Caught }\end{array}$ \\
\hline $\begin{array}{l}8^{\circ} 43^{1} \mathrm{~N} \\
155^{\circ} 50 \mathrm{~N}\end{array}$ & $\begin{array}{l}\text { May } \\
\frac{17}{65}\end{array}$ & $\begin{array}{l}\text { Pacifice } \\
\text { Ocean }\end{array}$ \\
\hline
\end{tabular}

Parasitic Animals:

Squid $\mid$\begin{tabular}{c|c|c}
$\begin{array}{c}\text { Squid } \\
\text { Beaks }\end{array}$ & Fish & $\begin{array}{c}\text { Fish } \\
\text { Remains }\end{array}$ \\
\hline I4 & +
\end{tabular}

Sula dactylatra

SOSC REF. NO. 204 POBSP. NO. 8

Prosic Animals:

Notes on Squid:

lots: 4 fish remains

Notes on Fish:

General Notes:

The crustacea remains appear to be parts of an isopod.

PACIFIC OCEANOGRAPHIC BIOLOGICAL SURVEY PROGRAM

SMITHSONIAN OCEANOGRAPHIC SORTING CENTER—SMITHSONIAN INSTITUTION

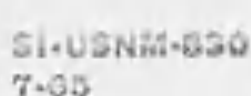

S. Willis $2 / 28 / 66$ crustacea remains

isopod

squid beaks
Other Animals
Remains Isopod (1)

$\mid$\begin{tabular}{c|c} 
Other \\
Crustacea \\
\hline Remimals \\
Isopod (2)
\end{tabular}

scuid beaks

\begin{tabular}{|c|c|c|c|c|c|c|c|c|c|}
\hline Sta. No. & \pm 086 & & Species & Pt & oma & externa & & $\begin{array}{l}\text { NO. } \\
\text { NO. }\end{array}$ & \\
\hline $\begin{array}{l}\text { Lat \& } \\
\text { Long. }\end{array}$ & Date & $\begin{array}{l}\text { Time } \\
\text { Caught }\end{array}$ & Squid & $\begin{array}{l}\text { Squid } \\
\text { Beaks }\end{array}$ & Fish & $\begin{array}{c}\text { Fish } \\
\text { Remains }\end{array}$ & $\begin{array}{l}\text { Parasitic } \\
\text { Animals }\end{array}$ & Crustacea & $\begin{array}{c}\text { Other } \\
\text { Animals }\end{array}$ \\
\hline $\begin{array}{l}8^{\circ} 43^{\circ} \mathrm{N} \\
155^{\circ} 50^{\circ}\end{array}$ & $\begin{array}{l}\text { Mey } \\
\frac{11}{65}\end{array}$ & $\begin{array}{l}\text { Pacific } \\
\text { Oceam }\end{array}$ & Rematnp & 296 & - & + & & & \\
\hline
\end{tabular}

Parasitic Animals:

Notes on Squid:

Iots: 3 squid remains

fish remains

squid beaks

Notes on Fish:

\author{
The fish remains are bones.
}

General Notes: 


$\begin{array}{llrl}\text { Sta. No. } & 1087 \text { Species Stercorarius Iongicauda } & \text { SOSC REF. NO. } & 204 \\ \text { POBSP. NO. } & 8\end{array}$

\begin{tabular}{|c|c|c|c|c|c|c|c|c|c|}
\hline $\begin{array}{l}\text { Lat \& } \\
\text { Long. }\end{array}$ & Date & $\begin{array}{l}\text { Time } \\
\text { Caught }\end{array}$ & Squid & $\begin{array}{l}\text { Squid } \\
\text { Beaks } \\
\end{array}$ & Fish & $\begin{array}{c}\text { Fish } \\
\text { Remains }\end{array}$ & $\begin{array}{l}\text { Parasitic } \\
\text { Animals }\end{array}$ & Crustacea & $\begin{array}{l}\text { Other } \\
\text { Animals }\end{array}$ \\
\hline $\begin{array}{l}8^{\circ} 40^{\prime} \mathrm{N} \\
155^{\circ} 54^{\circ} \\
\pi\end{array}$ & $\begin{array}{l}\text { May } \\
\frac{27}{65},\end{array}$ & $\begin{array}{l}\text { Pacific } \\
\text { Ocean }\end{array}$ & 1 & & & - & & & \\
\hline
\end{tabular}

lots: 2 squid

Notes on Squid: eggs

Notes on Fish:

Gencral Notes:

Besides the squid, the stomach contained only eggs which might be cephalopod eggs.

PACIFIC OCEANOGRAPHIC BIOLOGICAL SURVEY PROGRAM

SMITHSONIAN OCEANOGRAPHIC SORTING CENTER_SMITHISONIAN INSTITUTION 2/2I/66

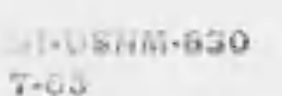

\begin{tabular}{|c|c|c|c|c|c|c|c|c|c|}
\hline Sta, No. & 2088 & & Specie: & Sul: & dacty & tra & & $\begin{array}{l}\text { NO. } \\
\text { NO. }\end{array}$ & \\
\hline $\begin{array}{l}\text { Lat \& } \\
\text { Long. }\end{array}$ & Date & $\begin{array}{l}\text { Time } \\
\text { Caught }\end{array}$ & Squid & $\begin{array}{l}\text { Squid } \\
\text { Beaks } \\
\end{array}$ & Fish & $\begin{array}{c}\text { Fish } \\
\text { Remains }\end{array}$ & $\begin{array}{l}\text { Parasitic } \\
\text { Animals } \\
\end{array}$ & Crustacea & $\begin{array}{l}\text { Other } \\
\text { Animals }\end{array}$ \\
\hline $\begin{array}{l}8^{\circ} 36^{\prime} \mathrm{N} \\
-155^{\circ} 59^{\circ} \\
7^{\prime}\end{array}$ & $\begin{array}{l}\text { May } \\
11, \\
65\end{array}$ & $\begin{array}{l}\text { Pacific } \\
\text { Ocean }\end{array}$ & & 2 & & + & & & \\
\hline
\end{tabular}

Notes on Squid:

Lots: 3 squid beaks

fish remains

digested remains

Notes on Fish:

The ish remains are bones.

General Notes:

The digested remains are beyond identification. 
Sta. No. $\quad 2089$

\begin{tabular}{|c|c|c|c|c|c|c|}
\hline $\begin{array}{l}\text { Lat \& } \\
\text { Long. }\end{array}$ & Date & $\begin{array}{l}\text { Time } \\
\text { Caught }\end{array}$ & Squid & $\begin{array}{l}\text { Squid } \\
\text { Beaks }\end{array}$ & Fish & $\begin{array}{c}\text { Fish } \\
\text { Remains }\end{array}$ \\
\hline $\begin{array}{l}8^{\circ} 33^{\circ} \mathrm{N} \\
156^{\circ} 14^{\circ} \\
4\end{array}$ & $\begin{array}{l}\text { May } \\
\frac{117}{65}\end{array}$ & $\begin{array}{l}\text { Pacific } \\
\text { Ocean }\end{array}$ & & 2 & 1 & + \\
\hline
\end{tabular}

Parasitic Animals:

lotes on Squid:

Notes on Fish:

1 fish tail is in the fish remains.

General Notes:

PACIFIC OCEANOGRAPHIC BIOLOGICAL SURVEY PROGRAM

SMITHSONIAN OCEANOGRAPHIC SORTING CENTER—SMITHISONIAN INSTITUTION

SI-USNM- 330
S. Wi.tuis $1 / 26 / 66$

lots: 4 squid beaks

fish

fish remains debris
Other Animals

\begin{tabular}{|c|c|c|c|c|c|c|c|c|c|}
\hline Sta. No. & 1090 & & Speci & Ste & fus & & & $\begin{array}{l}\text { NO. } \\
\text { NO. }\end{array}$ & \\
\hline $\begin{array}{l}\text { Lat \& } \\
\text { Long. }\end{array}$ & Date & $\begin{array}{l}\text { Time } \\
\text { Caught }\end{array}$ & Squid & $\begin{array}{l}\text { Squid } \\
\text { Beaks }\end{array}$ & Fish & $\begin{array}{c}\text { Fish } \\
\text { Remains }\end{array}$ & $\begin{array}{l}\text { Parasitic } \\
\text { Animals }\end{array}$ & Crustacea & $\begin{array}{l}\text { Other } \\
\text { Animals }\end{array}$ \\
\hline $\begin{array}{l}8^{\circ} 33^{\circ} \mathrm{N} \\
156^{\circ} 14^{\circ} \\
4\end{array}$ & $\begin{array}{l}\text { May } \\
\frac{11}{65} \text {, }\end{array}$ & $\begin{array}{l}\text { Pacific } \\
\text { Occan }\end{array}$ & & & 1 & + & & & \\
\hline
\end{tabular}

Parasitic Animals:

Notes on Squid:

Lats: 2 fish

fish remains

Notes on Fish: Fish with head digested; fish remains are mostly bones.

General Notes: 


\begin{tabular}{|c|c|c|c|c|c|c|c|c|c|}
\hline $\begin{array}{l}\text { Tat \& } \\
\text { Long. }\end{array}$ & Date & $\begin{array}{l}\text { Time } \\
\text { Cauight }\end{array}$ & Squid & $\begin{array}{l}\text { Squid } \\
\text { Beaks }\end{array}$ & Fish & $\begin{array}{c}\text { Fish } \\
\text { Remains }\end{array}$ & $\begin{array}{l}\text { Parasitic } \\
\text { Animals }\end{array}$ & Crustacea & $\begin{array}{l}\text { Other } \\
\text { Animals }\end{array}$ \\
\hline $156^{\circ} 48^{\circ}$ & $\frac{\text { Nay }}{\frac{217}{65}}$ & $\begin{array}{l}\text { Pacific } \\
\text { Ocean }\end{array}$ & $\frac{1}{\text { Remains }}$ & 99 & & + & & & \\
\hline
\end{tabular}

Parasitic Animals:

Notes on Squid:

lots: 5 scuid

Notes on Fish:

\section{General Notes:}

The remains are beyond identification.

PACIFIC OCEANOGRAPHIC BIOLOGICAL SURVEY PROGRAM

SMITHSONIAN OCEANOGRAPHIC SORTING CENTER_-SMITHSONIAN INSTITUTION

Si-บENM-ล30

SOSC REF. NO. 204 POBSP. NO.

8

\begin{tabular}{|c|c|c|c|c|c|c|c|c|c|}
\hline Sta. No. & 1094 & & Species & Sula & acty? & & & NO. & 8 \\
\hline $\begin{array}{l}\text { Lat \& } \\
\text { Long. }\end{array}$ & Date & $\begin{array}{c}\text { Time } \\
\text { Caught }\end{array}$ & Squid & $\begin{array}{l}\text { Squid } \\
\text { Beaks }\end{array}$ & Fish & $\begin{array}{c}\text { Fish } \\
\text { Remains }\end{array}$ & $\begin{array}{l}\text { Parasitic } \\
\text { Animals }\end{array}$ & Crustacea & $\begin{array}{l}\text { Other } \\
\text { Animals }\end{array}$ \\
\hline $\begin{array}{l}8^{\circ} 26^{\circ} N \\
156^{\circ} 43^{\circ} \\
W^{\circ}\end{array}$ & $\begin{array}{l}\text { May } \\
\frac{11}{65}\end{array}$ & $\begin{array}{l}\text { Pacific } \\
\text { Ocean }\end{array}$ & Remains & 10 & 2 & + & & & Isopod (I) \\
\hline
\end{tabular}

Parasitic Animals:

lots: 5 squid remains squid beaks

Notes on Squid:

Squid remains consist of eyes and bits of squid. isopod fish

fish remains

Notes on Fish:

The fish are digested.

General Notes: 
Sta. No. 1095

Species Sula dactylatra

\begin{tabular}{|c|c|c|c|c|c|c|c|}
\hline $\begin{array}{l}\text { Lat \& } \\
\text { Long. }\end{array}$ & Dute & $\begin{array}{l}\text { Time } \\
\text { Caught }\end{array}$ & Squid & $\begin{array}{l}\text { Squid } \\
\text { Beaks }\end{array}$ & Fish & $\begin{array}{c}\text { Fish } \\
\text { Remains }\end{array}$ & $\begin{array}{l}\text { Parasitic } \\
\text { Animals }\end{array}$ \\
\hline 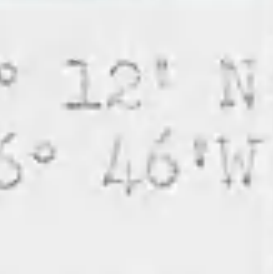 & $\frac{\text { Mey }}{65}$, & $\begin{array}{l}\text { Pactifio } \\
\text { Ocean }\end{array}$ & Remains & 23 & 2 & + & \\
\hline
\end{tabular}

Parasitic Animals:

The parasitic copepod had thwee distinct body parts.

\begin{tabular}{ll} 
Notes on Squid: & $\begin{array}{l}\text { squid beaks } \\
\text { fish } \\
\text { fish remains }\end{array}$ \\
\hline Notes on Fish: he fish were in a separate bag and are partially & $\begin{array}{l}\text { parcistic copepod } \\
\text { digested. }\end{array}$
\end{tabular}

General Notes:

PACIFIC OCEANOGRAPHIC BIOLOGICAL SURVEY PROGRAM

SMITHSONIAN OCEANOGRAPHIC SORTING CENTER—SMITHSONIAN INSTITUTION

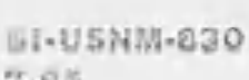

SMITHSONIAN OCEANOGRAPHIC SORIING CENTER - SMITHSONIAN INSTITUHION

S. Wi17is $2 / 28 / 66$

$7 \cdot 0$

\begin{tabular}{|c|c|c|}
\hline $\begin{array}{l}\text { Lat \& } \\
\text { Long. }\end{array}$ & Date & $\begin{array}{l}\text { Time } \\
\text { Caught }\end{array}$ \\
\hline $\begin{array}{l}5072: N \\
156^{\circ} 46^{\circ} \\
I\end{array}$ & $\frac{\text { May }}{65}$ & $\begin{array}{l}\text { Pacific } \\
\text { Ocean }\end{array}$ \\
\hline
\end{tabular}

Parasitic Animals:

Notes on Squid:

Species Sula dactylatra

\begin{tabular}{|c|c|c|c|} 
Squid & $\begin{array}{c}\text { Squid } \\
\text { Beaks }\end{array}$ & Fish & $\begin{array}{c}\text { Fish } \\
\text { Remains }\end{array}$ \\
\hline 3 & 2 & + \\
\hline & & +
\end{tabular}


SOSC REF. NO. 204

Sta. No. 2098

Species Sula dactylatra

POBSP. NO.

\begin{tabular}{|c|c|c|c|c|c|c|c|c|c|}
\hline $\begin{array}{l}\text { Lat \& } \\
\text { Long. }\end{array}$ & Date & Time & Squid & $\begin{array}{l}\text { Squid } \\
\text { Beaks }\end{array}$ & Fish & $\begin{array}{c}\text { Fish } \\
\text { Remains }\end{array}$ & $\begin{array}{l}\text { Parasitic } \\
\text { Animals }\end{array}$ & Crustacea & $\begin{array}{l}\text { Other } \\
\text { Animals }\end{array}$ \\
\hline $\begin{array}{l}3^{\circ} 12^{8} N \\
156^{\circ} \quad 46^{\circ} \\
W\end{array}$ & $\begin{array}{l}\text { Nay } \\
\frac{11}{65}\end{array}$ & Ocean & & 2 & 2 & $\uparrow$ & & & \\
\hline
\end{tabular}

Parasitic Animals:

Notes on Squid:

lots: 3 squid beaks fish

fish remains

Notes on Fish:

The fish are digested.

General Notes:

PACIFIC OCEANOGRAPHIC BIOLOGICAL SURVEY PROGRAM

SMITHSONIAN OCEANOGRAPHIC SORTING CENTER—SMITHSONIAN INSTITUTION

$\begin{array}{ll}\text { Si.USNT6.630 } & \text { S. Willis } 1 / 26 / 66\end{array}$

\begin{tabular}{|c|c|c|c|c|c|c|c|c|c|}
\hline Sta. No. & 2099 & & Specis & Sula & tcy la & & & $\begin{array}{l}\text { NO. } \\
\text { NO. }\end{array}$ & \\
\hline $\begin{array}{l}\text { Lat \& } \\
\text { Long. }\end{array}$ & Date & $\begin{array}{l}\text { Time } \\
\text { Caught }\end{array}$ & Squid & $\begin{array}{l}\text { Squid } \\
\text { Beaks } \\
\end{array}$ & Fish & $\begin{array}{c}\text { Fish } \\
\text { Remains }\end{array}$ & $\begin{array}{l}\text { Parasitic } \\
\text { Animals }\end{array}$ & Crustacea & $\begin{array}{l}\text { Other } \\
\text { Animals }\end{array}$ \\
\hline $\begin{array}{r}8^{\circ} \mathrm{OL}^{\circ} \mathrm{N} \\
156^{\circ} \quad 55^{\circ} \mathrm{W}\end{array}$ & $\frac{\text { Mas }}{\frac{77}{65}}$ & $\begin{array}{l}\text { Pacific } \\
\text { Ocean }\end{array}$ & 2 & 28 & 1 & + & & & \\
\hline
\end{tabular}

Parasitic Animals:

Notes on Squid:

The squid have detached tentacles.

lots: Li squid

squid beaks

fish

Notes on Fish:

fish remains

The fish is digested.

General Notes: 
Sta. No. 1091

\begin{tabular}{|c|c|c|c|}
\hline $\begin{array}{l}\text { Lat \& } \\
\text { Long. }\end{array}$ & Date & $\begin{array}{l}\text { Time } \\
\text { Caught }\end{array}$ & Squid \\
\hline $\begin{array}{l}8^{\circ} 29^{\prime} \mathrm{N} \\
156^{\circ} 25^{\circ} \\
W\end{array}$ & $\begin{array}{l}\text { May } \\
\frac{77}{65},\end{array}$ & $\begin{array}{l}\text { Pacific } \\
\text { Ocean }\end{array}$ & \\
\hline
\end{tabular}

Parasitic Animals:

Notes on Squid:

Notes on Fish:

General Notes:

The debris is a small lead ball.

lots: 4 fish remains squid beaks

digested remains debris
Other Animals

\section{PACIFIC OCEANOGRAPHIC BIOLOGICAL SURVEY PROGRAM
SMITHSONIAN OCEANOGRAPHIC SORTING CENTER—SMITHSONIAN INSTITUTION W. WiI $1 / 26 / 66$ \\ PACIFIC OCEANOGRAPHIC BIOLOGICAL SURVEY PROGRAM
SMITHSONIAN OCEANOGRAPHIC SORTING CENTER—SMITHSONIAN INSTITUTION S. Wi1.2is $1 / 26 / 66$

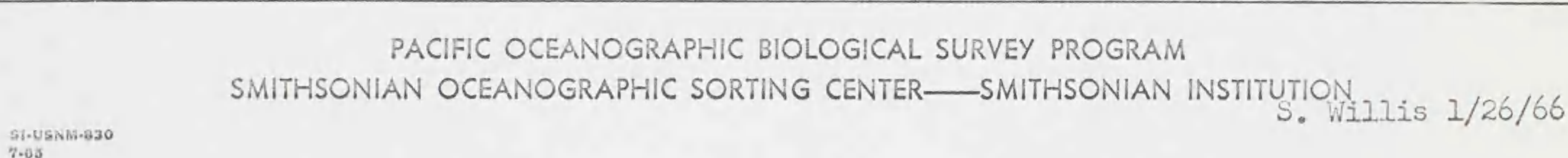

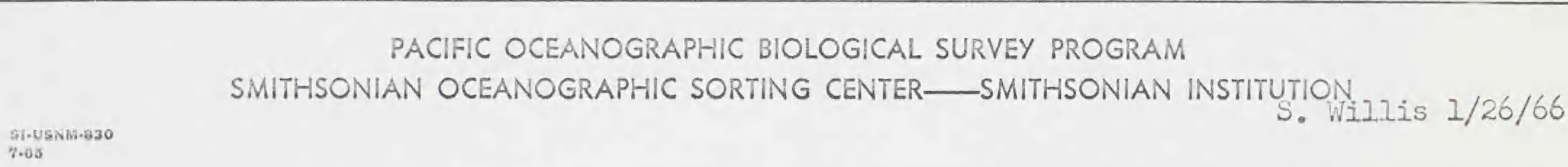

SOSC REF. NO. POBSP. NO.

204

8

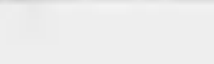

$+2$

\begin{tabular}{|c|c|c|c|c|c|c|c|c|c|}
\hline \multirow{2}{*}{$\begin{array}{l}\text { Sta. No. } \\
\text { Lat \& } \\
\text { Long. }\end{array}$} & \multicolumn{2}{|c|}{1092} & Species & \multicolumn{3}{|c|}{ Sterna fuscats } & \multicolumn{2}{|c|}{$\begin{array}{c}\text { SOSC REF. NO. } \\
\text { POBSP. NO. }\end{array}$} & \multirow[b]{2}{*}{$\begin{array}{l}\text { Other } \\
\text { Animals }\end{array}$} \\
\hline & Date & $\begin{array}{l}\text { Time } \\
\text { Caught }\end{array}$ & Squid & $\begin{array}{l}\text { Squid } \\
\text { Beaks }\end{array}$ & Fish & $\begin{array}{c}\text { Fish } \\
\text { Remains }\end{array}$ & $\begin{array}{l}\text { Parasitic } \\
\text { Animals }\end{array}$ & Crustacea & \\
\hline $\begin{array}{l}22^{\circ} 27^{\circ} \mathrm{NV} \\
256^{\circ} 25^{\circ} \mathrm{W}\end{array}$ & $\begin{array}{l}\text { May } \\
\frac{11}{65},\end{array}$ & $\begin{array}{l}\text { Pacific } \\
\text { Ocean }\end{array}$ & & & & + & & & \\
\hline
\end{tabular}

Parasitic Animals:

lots: I fish remains

\begin{abstract}
Notes on Squid:
\end{abstract}

\title{
Notes on Fish:
}

General Notes: 


\begin{tabular}{|c|c|c|c|c|c|c|c|c|c|}
\hline Sta. No. & 11200 & & Species & Si & dac & tra & & $\begin{array}{l}\text { NO. } \\
\text { No. }\end{array}$ & \\
\hline $\begin{array}{l}\text { Lat \& } \\
\text { Long. }\end{array}$ & Date & $\begin{array}{l}\text { Time } \\
\text { Caught }\end{array}$ & Squid & $\begin{array}{l}\text { Squid } \\
\text { Beaks }\end{array}$ & Fish & $\begin{array}{c}\text { Fish } \\
\text { Remains }\end{array}$ & $\begin{array}{l}\text { Parasitic } \\
\text { Animals } \\
\end{array}$ & Crustacea & $\begin{array}{l}\text { Other } \\
\text { Animals }\end{array}$ \\
\hline $\begin{array}{l}8^{\circ} 03^{*} \mathrm{~N} \\
257^{\circ} \text { of }\end{array}$ & $\begin{array}{l}\text { May } \\
112 \\
65\end{array}$ & $\begin{array}{l}\text { Pacific } \\
\text { Ocean }\end{array}$ & Remains & 19 & & + & $\begin{array}{r}\text { Tapeworms } \\
(5)\end{array}$ & & \\
\hline
\end{tabular}

Parasitic Animals:

The tapewornis were approximately $5-10 \mathrm{~cm}$. Iong.

Notes on Squid:

Iots: 4 squid remains

squid beaks

tapeworms

fish remains

Notes on Fish:

General Notes:

PACIFIC OCEANOGRAPHIC BIOLOGICAL SURVEY PROGRAM

SMITHSONIAN OCEANOGRAPHIC SORTING CENTER—SMITHSONIAN INSTITUTION

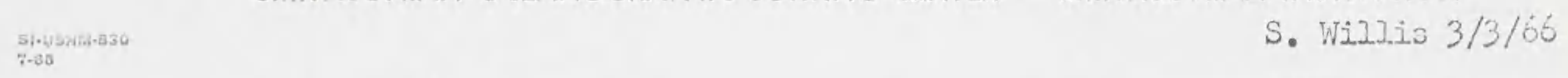

SOSC REF. NO. 204

Sta. No. $\quad 1101$

Species Stema fuscata POBSP. NO. 8

\begin{tabular}{|c|c|c|c|c|c|c|c|c|c|}
\hline $\begin{array}{l}\text { Lat \& } \\
\text { Long. }\end{array}$ & Date & $\begin{array}{l}\text { Time } \\
\text { Caught }\end{array}$ & Squid & $\begin{array}{l}\text { Squid } \\
\text { Beaks }\end{array}$ & Fish & $\begin{array}{c}\text { Fish } \\
\text { Remains }\end{array}$ & $\begin{array}{l}\text { Parasitic } \\
\text { Animals }\end{array}$ & Crustacea & $\begin{array}{l}\text { Other } \\
\text { Animals }\end{array}$ \\
\hline $\begin{array}{l}8^{\circ} 03^{\circ} \mathrm{N} \\
15^{\circ} 07^{\circ} \\
4^{\circ}\end{array}$ & $\begin{array}{l}\text { Nay } \\
\frac{11}{65}\end{array}$ & $\begin{array}{l}\text { Pacific } \\
\text { Ocean }\end{array}$ & & & 2 & + & & & \\
\hline
\end{tabular}

Parasitic Animals:

Iots: 2 fish

Notes on Squid:

Iish remains

Notes on Fish:

Two fish tails are in the fish remains.

General Notes: 


\begin{tabular}{|c|c|c|c|c|c|c|c|c|c|}
\hline $\begin{array}{l}\text { Lat \& } \\
\text { Long. }\end{array}$ & Date & $\begin{array}{l}\text { Time } \\
\text { Caught }\end{array}$ & Squid & $\begin{array}{l}\text { Squid } \\
\text { Beaks }\end{array}$ & Fish & $\begin{array}{c}\text { Fish } \\
\text { Remains } \\
\end{array}$ & $\begin{array}{l}\text { Parasitic } \\
\text { Animals }\end{array}$ & Crustacea & $\begin{array}{c}\text { Other } \\
\text { Animals }\end{array}$ \\
\hline $\begin{array}{c}0.03 \cdot \mathrm{N} \\
57^{\circ} 07 \cdot \mathrm{N}\end{array}$ & $\frac{11}{65}$ & $\begin{array}{l}\text { Pacific } \\
\text { Ocean }\end{array}$ & ${ }^{7}$ & 96 & & - & & & \\
\hline
\end{tabular}

Parasitic Animals:

Notes on Squid: AII the squid have detached tentacles.

Iotsi 5 squids

squid beaks

digested remains

squid remains

Notes on Fish:

$\operatorname{rock}(1)$

General Notes: 1. The rock is mediun size and flotable.

2. Several wood particles were left in the digested remains.

PACIFIC OCEANOGRAPHIC BIOLOGICAL SURVEY PROGRAM

SMITHSONIAN OCEANOGRAPHIC SORTING CENTER_-SMITHSONIAN INSTITUTION

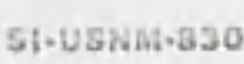

S. Willis $2 / 28 / 66$

Sta. No. 1203

Species Sula dactylatra

SOSC REF. NO. 204 POBSP. NO. 8

\begin{tabular}{|c|c|c|c|c|c|c|c|c|c|}
\hline $\begin{array}{l}\text { Lat \& } \\
\text { Long. }\end{array}$ & Date & $\begin{array}{l}\text { Time } \\
\text { Caught }\end{array}$ & Squid & $\begin{array}{l}\text { Squid } \\
\text { Beaks }\end{array}$ & Fish & $\begin{array}{c}\text { Fish } \\
\text { Remains }\end{array}$ & $\begin{array}{l}\text { Parasitic } \\
\text { Animals }\end{array}$ & Crustacea & $\begin{array}{l}\text { Other } \\
\text { Animals }\end{array}$ \\
\hline $\begin{array}{l}3^{\circ} 03^{\circ} \mathrm{N} \\
157^{\circ} 08^{\circ} \mathrm{W}\end{array}$ & $\begin{array}{l}\text { Hay } \\
\frac{37}{65},\end{array}$ & $\begin{array}{l}\text { Pacific } \\
\text { Coean }\end{array}$ & 4 & 22 & 2 & $\phi$ & & & \\
\hline
\end{tabular}

Parasitic Animals:

Lots: 4. squid

Notes on Squid: The squid have detached tentacles.

squid beaks fish

fish remains

Notes on Fish: The head of one fish is digested.

General Notes: 


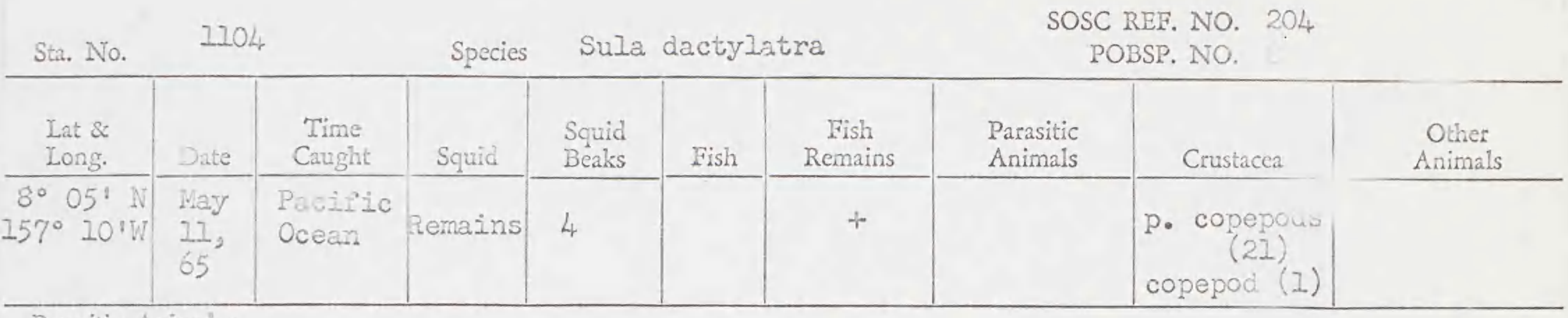

Parasitic Animals:

lots: 5 squid remains

Notes on Squid:

squid beaks

fish remains

Notes on Fish:

parasitic copepods

non-parasitic copepod

General Notes:

Most of the parasitic copepods had three (3) distinct body parts.

PACIFIC OCEANOGRAPHIC BIOLOGICAL SURVEY PROGRAM

SMITHSONIAN OCEANOGRAPHIC SORTING CENTER_SMITHSONIAN INSTITUTION

Si.USNM-OSO

$t .62$
$5 i \cdot 42 \pi$

S. Willis $3 / 2 / 66$

Sta. No. $\quad 2106$

Species Sterna fuscata

SOSC REF. NO. 204

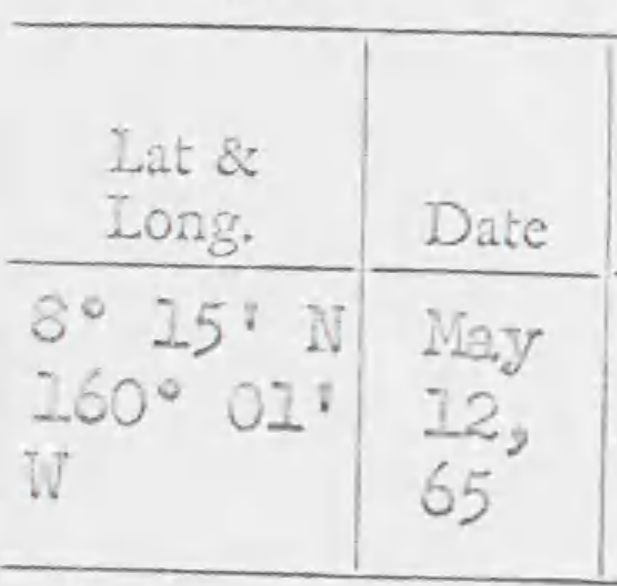

\begin{tabular}{l} 
Time \\
Caught \\
\hline $\begin{array}{l}\text { Pacific } \\
\text { Ocean }\end{array}$
\end{tabular}

\begin{tabular}{|l|l|c|}
$\begin{array}{l}\text { Squid } \\
\text { Beaks }\end{array}$ & Fish & $\begin{array}{c}\text { Fish } \\
\text { Remains }\end{array}$ \\
\hline & & - \\
&
\end{tabular}

POBSP. NO.

8

Parasitic Animals:

Notes on Squid:

lots: 1 digested remains

Notes on Fish:

General Notes:

The remains are beyond identification.

PACIFIC OCEANOGRAPHIC BIOLOGICAL SURVEY PROGRAM

SMITHSONIAN OCEANOGRAPHIC SORTING CENTER—SMITHSONIAN INSTITUTION 
Sta. No. 1207

\begin{tabular}{|c|c|c|c|c|c|c|}
\hline $\begin{array}{l}\text { Lat \& } \\
\text { Long. }\end{array}$ & Date & $\begin{array}{l}\text { Time } \\
\text { Caught }\end{array}$ & Squid & $\begin{array}{l}\text { Squid } \\
\text { Beaks }\end{array}$ & Fish & $\begin{array}{c}\text { Fish } \\
\text { Remains }\end{array}$ \\
\hline $\begin{array}{l}8^{\circ} 15^{\circ} \mathrm{N} \\
160^{\circ} 01^{?} \\
N\end{array}$ & $\begin{array}{l}\text { 1.2y } \\
\frac{22}{65}\end{array}$ & $\begin{array}{l}\text { Protific } \\
\text { pcesal }\end{array}$ & 1 & & & + \\
\hline
\end{tabular}

Parasitic Animals:

Notes on Scuid:
SOSC REF. NO. 204 POBSP. NO. 8

Squid has tentacles detached.

Notes on Fish:

General Notes:

PACIFIC OCEANOGRAPHIC BIOLOGICAL SURVEY PROGRAM

SMITHSONIAN OCEANOGRAPHIC SORTING CENTER—SMITHSONIAN INSTITUTION

Si-USNM-2030

Other Arimals

$\frac{\text { lots: } 2 \text { squid }}{\text { fish remains }}$

\begin{tabular}{l|l}
$\begin{array}{c}\text { Parasitic } \\
\text { Animals }\end{array}$ & Crustacea \\
\hline & $\begin{array}{c}\text { Other } \\
\text { Animals }\end{array}$ \\
\hline
\end{tabular}

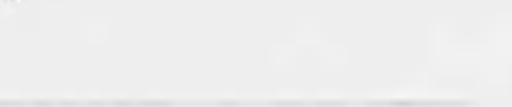

Sta. No. 1109

Species Sula dactylatra

SOSC REF. NO. 204 POBSP. NO. 8

\begin{tabular}{|c|c|c|c|c|c|c|c|c|c|}
\hline $\begin{array}{l}\text { Lat \& } \\
\text { Long. }\end{array}$ & Date & $\begin{array}{l}\text { Time } \\
\text { Caught }\end{array}$ & Squid & $\begin{array}{l}\text { Squid } \\
\text { Beaks }\end{array}$ & Fish & $\begin{array}{l}\text { Fish } \\
\text { Remains }\end{array}$ & $\begin{array}{l}\text { Parasitic } \\
\text { Animals }\end{array}$ & Crustacea & $\begin{array}{l}\text { Other } \\
\text { Animals }\end{array}$ \\
\hline $\begin{array}{l}8013: N \\
160^{\circ} 26^{\circ} \\
N\end{array}$ & $\begin{array}{l}\text { May } \\
\frac{12}{65}\end{array}$ & $\begin{array}{l}\text { Pacific } \\
\text { Ocean }\end{array}$ & $\begin{array}{c}10 \\
\text { Remaind }\end{array}$ & 11 & 3 & + & & & \\
\hline
\end{tabular}

Parasitic Animals:

Notes on Squid: I. 4 squid have no tentacles.

2. 5 squid eyes are in the squid remains.

Notes on Fish:

The fish heads are digested.
Iots: 5 squid

squid remains

squid beaks

fish

fish remains

General Notes: 
Sta. No. 1220 Species Puffinus pacificus

\begin{tabular}{l} 
Time \\
Caught \\
\hline Paciftic \\
Ocean
\end{tabular}

Parasitic Animais:

\begin{tabular}{|l|l|l|c|} 
Squid & $\begin{array}{c}\text { Squid } \\
\text { Beaks }\end{array}$ & Fish & $\begin{array}{c}\text { Fish } \\
\text { Remains }\end{array}$ \\
\hline 1 & & & - \\
& & & \\
& &
\end{tabular}

\begin{tabular}{|c|} 
Parasitic \\
Animals \\
\hline
\end{tabular}

SOSC REF. NO. 204 POBSP. NO.

8

\begin{tabular}{|c|c|} 
Crustacea & $\begin{array}{c}\text { Other } \\
\text { Animals }\end{array}$ \\
\hline & \\
& \\
\hline
\end{tabular}

lots: 2 squid

Notes on Scuid: digested remains

Notes on Fin

Guneral Notes:

PACIFIC OCEANOGRAPHIC BIOLOGICAL SURVEY PROGRAM SMITHSONIAN OCEANOGRAPHIC SORTING CENTER—SMITHSONIAN INSTITUTION

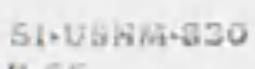

$7 \cdot 63$

\begin{tabular}{|c|c|c|c|c|c|c|c|c|c|}
\hline Sta. No. & 1111 & & Speci & & dac & atra & & $\begin{array}{l}\text { NO. } \\
\text { NO. }\end{array}$ & \\
\hline $\begin{array}{l}\text { Lat \& } \\
\text { Long. }\end{array}$ & Date & $\begin{array}{l}\text { Time } \\
\text { Caught }\end{array}$ & Squid & $\begin{array}{l}\text { Squid } \\
\text { Beaks }\end{array}$ & Fish & $\begin{array}{c}\text { Fish } \\
\text { Remains }\end{array}$ & $\begin{array}{l}\text { Parasitic } \\
\text { Animals } \\
\end{array}$ & Crustacea & $\begin{array}{l}\text { Other } \\
\text { Animals }\end{array}$ \\
\hline $\begin{array}{l}8^{\circ} 12^{\prime} \mathrm{N} \\
160^{\circ} 59^{\circ} \\
0\end{array}$ & $\begin{array}{l}\text { Wy } \\
\frac{12}{65},\end{array}$ & $\begin{array}{l}\text { Pacinic } \\
\text { Ocean }\end{array}$ & & & 3 & + & & & \\
\hline
\end{tabular}

Parasitic Animals:

lots: 2 fish

Notes on Squid:

fish remains

General Notes: 


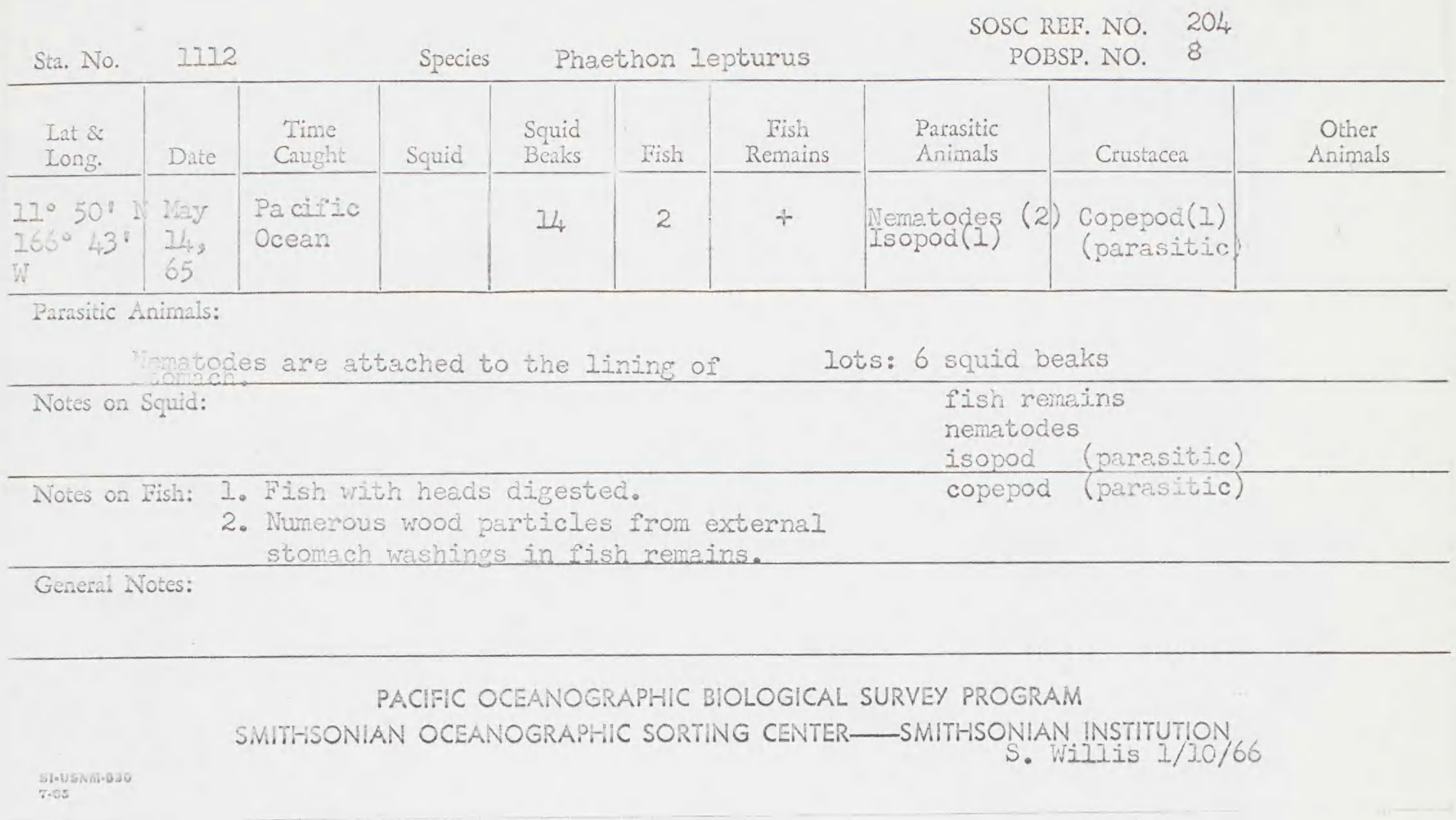

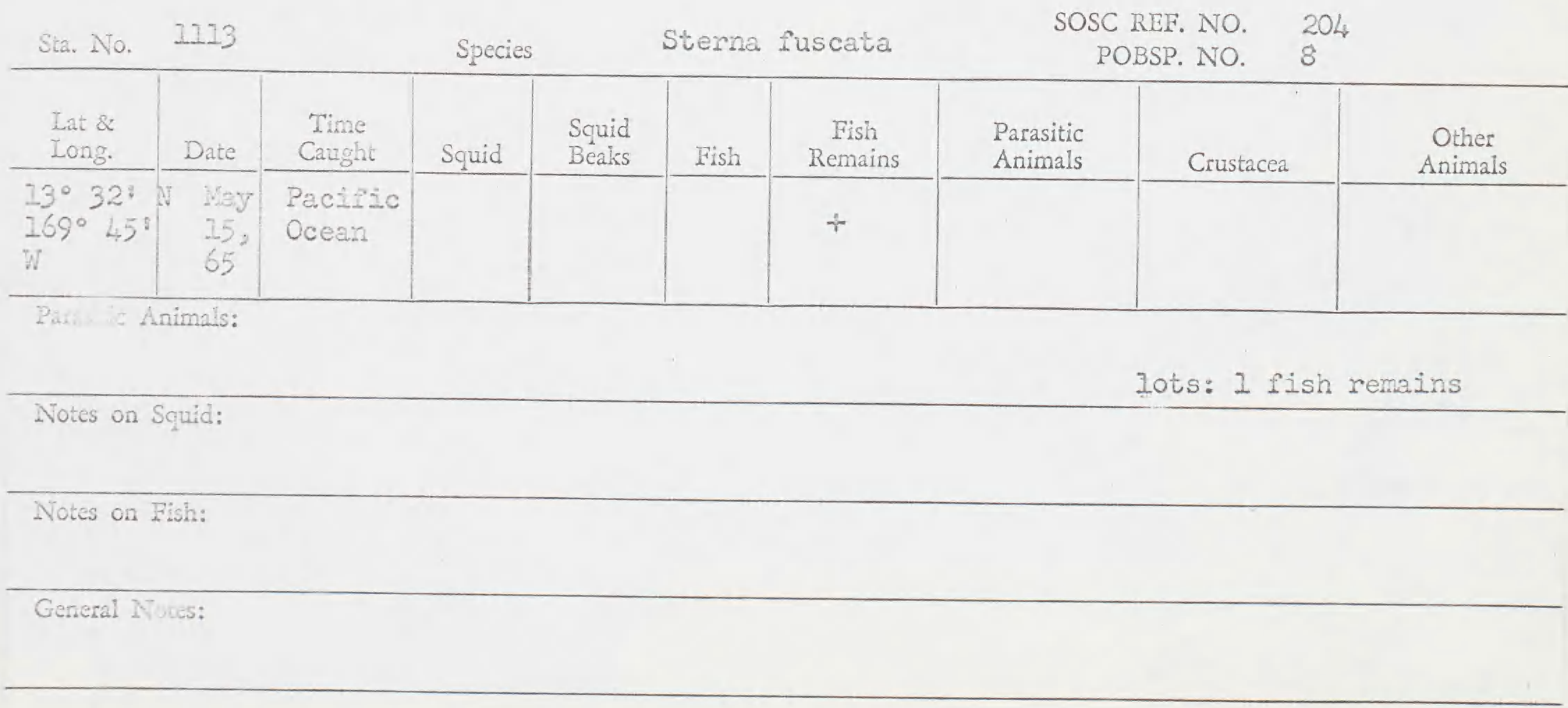


Sta. No. 2115 Species

Sterna fuscata

SOSC REF. NO.

POBSP. NO.

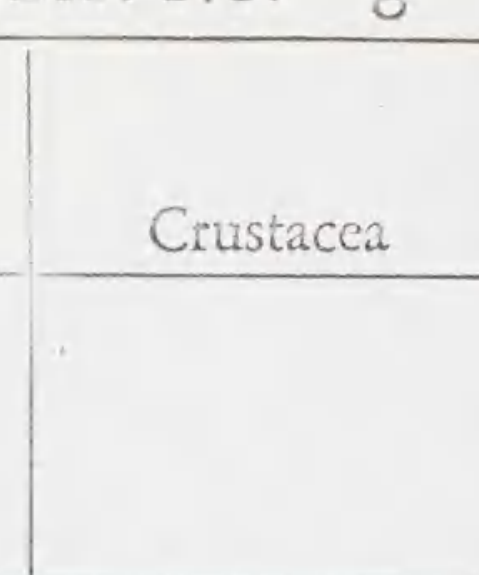

Parasitic Animals:

\begin{tabular}{|c|c|c|c|c|}
\hline $\begin{array}{l}\text { Tine } \\
\text { Cotught }\end{array}$ & Souid & $\begin{array}{l}\text { Squid } \\
\text { Beaks }\end{array}$ & Fish & $\begin{array}{c}\text { Fish } \\
\text { Remains }\end{array}$ \\
\hline $\begin{array}{l}\text { Pacific } \\
\text { Ocean }\end{array}$ & & & & - \\
\hline
\end{tabular}

\begin{tabular}{l} 
Parasitic \\
Animals \\
\hline
\end{tabular}

204 8

Iots: 1 digested remains

Notes on Squid:

Notes on Fish:

General Notes:

The remains are beyond identification.

PACIFIC OCEANOGRAPHIC BIOLOGICAL SURVEY PROGRAM

SMITHSONIAN OCEANOGRAPHIC SORTING CENTER-SMITHSONIAN INSTITUTION

S. Willis $1 / 10 / 66$

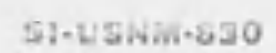

$7+00$

SOSC REF. NO. 204

Sta. No. $\quad 2116$

Species

Phaethon lepturus

POBSP. NO.

8

\begin{tabular}{|c|c|c|c|c|c|c|c|c|c|}
\hline $\begin{array}{l}\text { Lat \& } \\
\text { Long. }\end{array}$ & Date & $\begin{array}{l}\text { Time } \\
\text { Caught }\end{array}$ & Squid & $\begin{array}{l}\text { Squid } \\
\text { Beaks }\end{array}$ & Fish & $\begin{array}{c}\text { Fish } \\
\text { Remains }\end{array}$ & $\begin{array}{l}\text { Parasitic } \\
\text { Animals }\end{array}$ & Crustacea & $\begin{array}{l}\text { Other } \\
\text { Animals }\end{array}$ \\
\hline $\begin{array}{ll}3^{\circ} & 55^{\circ} \mathrm{N} \\
0^{\circ} & 34^{\circ} \mathrm{W}\end{array}$ & $\begin{array}{l}\text { May } \\
16, \\
65\end{array}$ & $\begin{array}{l}\text { Pacific } \\
\text { Ocoan }\end{array}$ & Remains & 11 & 4 & + & & & \\
\hline
\end{tabular}

Parasitic Animals:

lots: 4 squid remains

squid beaks

fish remains

Notes on Squid: One group of tentacles is in the remains. fish

\footnotetext{
Notes on Fish: The fish are partially digested.
}

\footnotetext{
General Notes:
} 
Sta. No. $\quad 2143$

Sterna fuscata

SOSC REF. NO. POBSP. NO. 8

204

Species

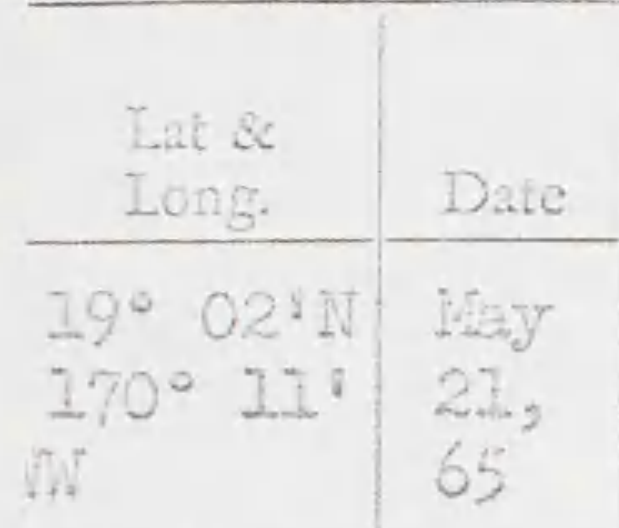

\begin{tabular}{|l|l|l|l|c|}
\begin{tabular}{c|c} 
Time \\
Caught
\end{tabular} & Squid & $\begin{array}{c}\text { Squid } \\
\text { Beaks }\end{array}$ & Fish & $\begin{array}{c}\text { Fish } \\
\text { Remains }\end{array}$ \\
\cline { 1 - 3 } $\begin{array}{l}\text { Pacific } \\
\text { Ocean }\end{array}$ & & & & - \\
& & & -
\end{tabular}

\begin{tabular}{|l|l|l}
$\begin{array}{c}\text { Parasitic } \\
\text { Animals }\end{array}$ & Crustacea & $\begin{array}{c}\text { Other } \\
\text { Animals }\end{array}$ \\
\hline & & \\
& & \\
&
\end{tabular}

Purusitic Animals:

Notes on Squid:

Iotsi I digested remains

Notes on Fish:

General Notes:

The remains are beyond identification.

PACIFIC OCEANOGRAPHIC BIOLOGICAL SURVEY PROGRAM

SMITHSONIAN OCEANOGRAPHIC SORTING CENTER—SMITHSONIAN INSTITUTION

SL-บธKพิ-850

7. 63

S. Willis $3 / 3 / 66$

\begin{tabular}{|c|c|c|c|c|c|c|c|c|c|}
\hline Sta. No. & 124,4 & & Specic & & na 1 & ata & & $\begin{array}{l}\text { No. } \\
\text { NO. }\end{array}$ & \\
\hline $\begin{array}{l}\text { Lat \& } \\
\text { Long. }\end{array}$ & Date & $\begin{array}{l}\text { Time } \\
\text { Caught }\end{array}$ & Squid & $\begin{array}{l}\text { Squid } \\
\text { Beaks } \\
\end{array}$ & Fish & $\begin{array}{c}\text { Fish } \\
\text { Remains }\end{array}$ & $\begin{array}{l}\text { Parasitic } \\
\text { Animals } \\
\end{array}$ & Crustacea & $\begin{array}{l}\text { Other } \\
\text { Animals } \\
\end{array}$ \\
\hline $\begin{array}{l}29^{\circ} 02^{\circ} \\
270^{\circ} 11^{\circ} \%\end{array}$ & $\begin{array}{l}\text { May } \\
21, \\
65\end{array}$ & $\begin{array}{l}\text { Pacific } \\
\text { Ocean }\end{array}$ & & & & - & & & \\
\hline
\end{tabular}

Notes on Squid: lots: I digested remains

Notes on Fish:

General Notes:

The re ins are bend identification.

PACIFIC OCEANOGRAPHIC NOLOGICAL SURVEY PROGRAM

SMITHSONIAN OCEANOGRAPHIS TH STHER_SMITHSONIAM 
SOSC REF. NO. 204

Sta. No. $\quad 1149 \quad$ Species Sterna fuscata

POBSP. NO. 8

\begin{tabular}{|c|c|c|c|c|c|c|c|c|c|}
\hline $\begin{array}{l}\text { Lat \& } \\
\text { Long. }\end{array}$ & Dace & $\begin{array}{l}\text { Time } \\
\text { Caught }\end{array}$ & Squid & $\begin{array}{l}\text { Squid } \\
\text { Beaks }\end{array}$ & Fish & $\begin{array}{l}\text { Fish } \\
\text { Remains }\end{array}$ & $\begin{array}{l}\text { Parasitic } \\
\text { Animals }\end{array}$ & Crustacea & $\begin{array}{c}\text { Other } \\
\text { Animals }\end{array}$ \\
\hline $\begin{array}{l}20^{\circ} 59^{\circ} \mathrm{W} \\
168^{\circ} 04^{\circ} \\
W^{\circ}\end{array}$ & $\begin{array}{l}\text { May } \\
22, \\
6 \%\end{array}$ & $\begin{array}{l}\text { Pacif } \\
\text { Ocean }\end{array}$ & & & & - & & & \\
\hline
\end{tabular}

Parasitic Animals:

Lots: 0

Notes on Squid:

Notes on Fish:

General Notes:

The stomach was empty.

PACIFIC OCEANOGRAPHIC BIOLOGICAL SURVEY PROGRAM

SMITHSONIAN OCEANOGRAPHIC SORTING CENTER—SMITHSONIAN INSTITUTION

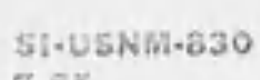

S. Willis $3 / 3 / 66$

$7 \cdot \overline{0}$

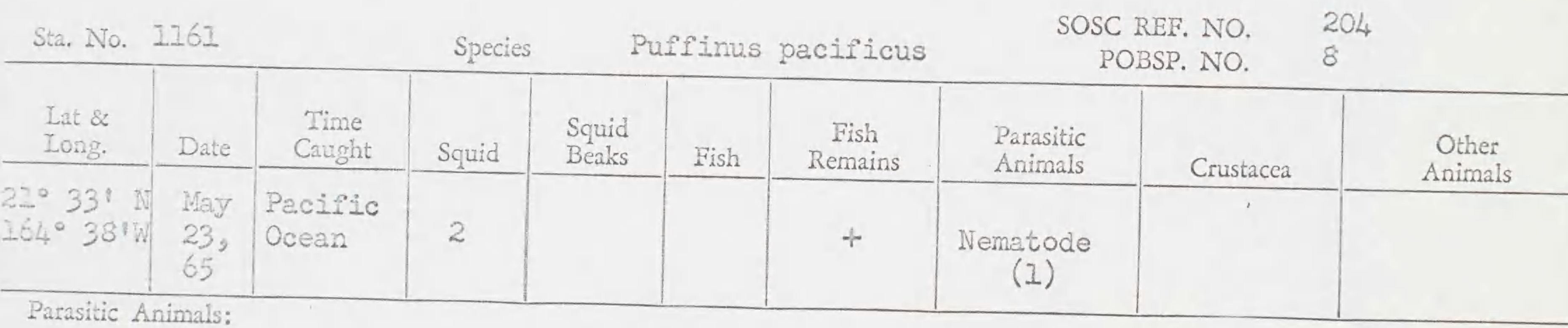

Notes on Squid: I squid has tentacles attached. The other has no tentacles and is in bad condition covered with saw dust. lots: 3 squids

fish remains nematode

Notes on Fish: 
SOSC REF. NO. 204

Sta. No. $1162 \quad$ Species Puffinus pacificus POBSP. NO. 8

\begin{tabular}{c|c|c|c|c|c|c|c|c}
\hline Lat 8 \\
Long.
\end{tabular}

Parasitic Animals:

Notes on Squid:

Iots: 4 squid beaks

fish remains

pelycepods

digested remains

Notes on Fish:

General Notes:

PACIFIC OCEANOGRAPHIC BIOLOGICAL SURVEY PROGRAM

SMITHSONIAN OCEANOGRAPHIC SORTING CENTER—SMITHISONIAN INSTITUTION

S1. USWN-030

$7-83$

S. Wizlis $3 / 3 / 66$

Sta. No. 1164

\begin{tabular}{|c|c|}
\hline $\begin{array}{l}\text { Lat \& } \\
\text { Long. }\end{array}$ & Date \\
\hline $\begin{array}{l}27^{\circ} 33^{\circ} \\
264^{\circ} \\
W\end{array}$ & $\begin{array}{l}\text { Nay } \\
23 \\
65\end{array}$ \\
\hline
\end{tabular}

Parasitic Animals:

Notes on Squid:

Species Sterna fuscata

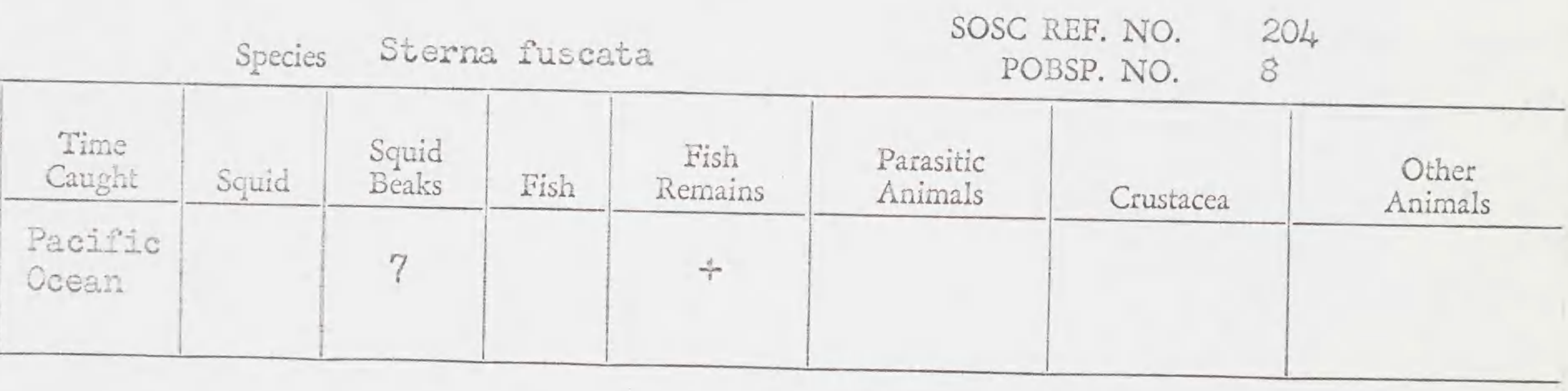

7ots: 3 squid beaks

fish remains

squid tentacles( 1 group)

Notes on Fish:

General Notes: 
SOSC REF. NO. ${ }_{8}^{204}$

Sta. No. 1165

Species Sterna fuscata

POBSP. NO.

\begin{tabular}{|c|c|c|c|c|c|c|c|c|c|}
\hline $\begin{array}{l}\text { Lat } s \\
\text { Long. }\end{array}$ & Date & $\begin{array}{l}\text { Time } \\
\text { Caught }\end{array}$ & Squid & $\begin{array}{l}\text { Squid } \\
\text { Beaks }\end{array}$ & Fish & $\begin{array}{l}\text { Fish } \\
\text { Remains }\end{array}$ & $\begin{array}{l}\text { Parasitic } \\
\text { Animals }\end{array}$ & Crustacea & $\begin{array}{l}\text { Other } \\
\text { Animals }\end{array}$ \\
\hline $\begin{array}{l}21^{\circ} 33^{\circ} \\
164^{\circ} 38^{\circ}\end{array}$ & $\begin{array}{l}\text { Wey } \\
23, \\
65\end{array}$ & $\begin{array}{l}\text { Pacifie } \\
\text { Oecan }\end{array}$ & 1 & 4 & 1 & + & & & \\
\hline
\end{tabular}

Pansitic Animals:

lots: 6 squid beaks

Notes on Squid: One group of tentacles in squid remains.

Notes on Fish: 1. 2 digested fish halves th the remains.

2. Fish with head digested.

General Notes:

Digested remains are beyond identification.

PACIFIC OCEANOGRAPHIC BIOLOGICAL SURVEY PROGRAM

SMITHSONIAN OCEANOGRAPHIC SORTING CENTER—SMITHSONIAN INSTITUTION

Si-cisanim-45o

$7 \cdot 65$

S. Willis $1 / 10 / 66$

\begin{tabular}{|c|c|c|c|c|c|c|c|c|c|}
\hline Sta. No. & $126 ?$ & & Species & & ufin & pacifici & & $\begin{array}{l}\text { NO. } 2 \\
\text { No. } 8 \\
\end{array}$ & \\
\hline $\begin{array}{l}\text { Lat \& } \\
\text { Long. }\end{array}$ & Date & $\begin{array}{l}\text { Time } \\
\text { Caught }\end{array}$ & Squid & $\begin{array}{l}\text { Squid } \\
\text { Beaks }\end{array}$ & Fish & $\begin{array}{c}\text { Fish } \\
\text { Remains } \\
\end{array}$ & $\begin{array}{l}\text { Parasitic } \\
\text { Animals }\end{array}$ & Crustacea & $\begin{array}{l}\text { Other } \\
\text { Animals }\end{array}$ \\
\hline $\begin{array}{l}21^{\circ} 33^{\circ} \mathrm{N} \\
264^{\circ} 30^{\circ} \\
\mathrm{N}\end{array}$ & $\begin{array}{l}\text { May } \\
23 \\
65\end{array}$ & $\begin{array}{l}\text { Pacilic } \\
\text { Ocean }\end{array}$ & femains & 144 & & + & & & \\
\hline
\end{tabular}

Notes on Sqquid:

2ots: 4 squid remains

squid beaks

fish remains

Notes on Fish:

The fifh remains are bones.

digested remans

General Notes: 
SOSC REF. NO. 204

Sta. No.

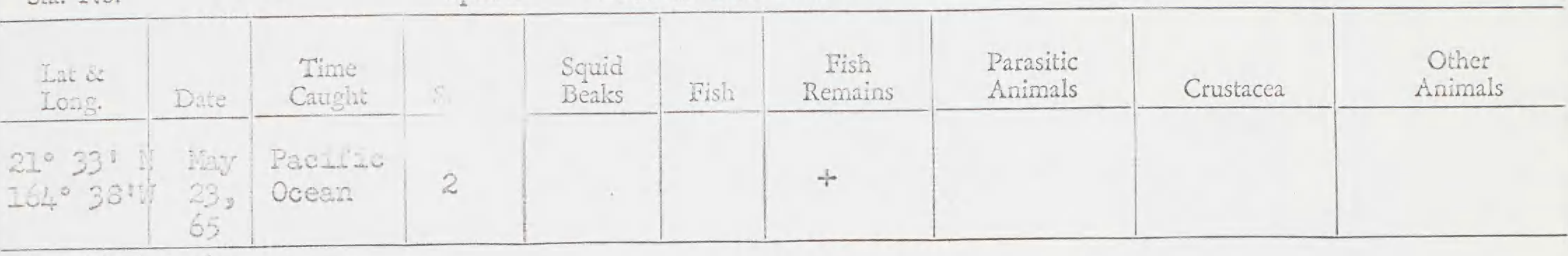

Parasitic Animals:

Lots: 2 squid

Notes on Squid:

Squid have tentacles attached.

fish remains

Notes on Fish:

General Notes:

No stomach was received just the contents.

PACIFIC OCEANOGRAPHIC BIOLOGICAL SURVEY PROGRAM

SMITHSONIAN OCEANOGRAPHIC SORTING CENTER—SMITHSONIAN INSTITUTION

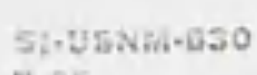

$7 \cdot 0.5$

S. Willis $3 / 3 / 66$

SOSC REF. NO. 204

Sta. No. $\quad 1171$

Species

Puffinus pacificus

POBSP. NO. 8

\begin{tabular}{|c|c|c|c|c|c|c|c|c|c|}
\hline $\begin{array}{l}\text { Lat \& } \\
\text { Long. }\end{array}$ & Date & $\begin{array}{l}\text { Time } \\
\text { Caught }\end{array}$ & Squid & $\begin{array}{l}\text { Squid } \\
\text { Beaks }\end{array}$ & Fish & $\begin{array}{c}\text { Fish } \\
\text { Remains }\end{array}$ & $\begin{array}{l}\text { Parasitic } \\
\text { Animals }\end{array}$ & Crustacea & $\begin{array}{l}\text { Other } \\
\text { Animals }\end{array}$ \\
\hline $\begin{array}{l}277^{\circ} 33^{\circ} \\
164^{\circ} \\
W^{\circ}\end{array}$ & $\begin{array}{l}\text { Ney } \\
23 \% \\
65\end{array}$ & $\begin{array}{l}\text { Pacific } \\
\text { Ocean }\end{array}$ & Remains & 88 & & $t$ & & & \\
\hline
\end{tabular}

Parasitic Animals:

lots: 4 squid remeins

squid beaks

Notes on Squid: Remains are several bits of digested squid. fish remains rock (1)

Notes on Fish:

General Notes:

The rock is medium size and flotable. 
SOSC REF. NO. 204

Sta. No. 1172

Species Pufinus pacificus

POBSP. NO.

8

\begin{tabular}{|c|c|c|c|c|c|c|c|c|c|}
\hline $\begin{array}{l}\text { Lat } 5 \text { Long. } \\
\text { Lon }\end{array}$ & Dute & $\begin{array}{l}\text { Time } \\
\text { Caught }\end{array}$ & Squid & $\begin{array}{l}\text { Squid } \\
\text { Beaks }\end{array}$ & Fish & $\begin{array}{c}\text { Fish } \\
\text { Remains }\end{array}$ & $\begin{array}{l}\text { Parasitic } \\
\text { Animals }\end{array}$ & Crustacea & $\begin{array}{l}\text { Other } \\
\text { Animals }\end{array}$ \\
\hline $\begin{array}{l}22^{\circ} 33^{\circ} 10 \\
164^{\circ} 35^{\circ} \\
W\end{array}$ & $\begin{array}{l}\text { May } \\
23,\end{array}$ & $\begin{array}{l}\text { Pacific } \\
\text { Occan }\end{array}$ & & 3 & & - & & & \\
\hline
\end{tabular}

Parasitic Animals:

lots: squid beaks

Notes on Squid:

digested remains

Notes on Fish:

General Notes:

PACIEIC OCEANOGRAPHIC BIOLOGICAL SURVEY PROGRAM

WTSONIAN OCEANOGRAPHIC SORTING CENTER_SMITHSONIAN INSTITUTION $3 / 3 / 66$

Si.uSmat.

7.05

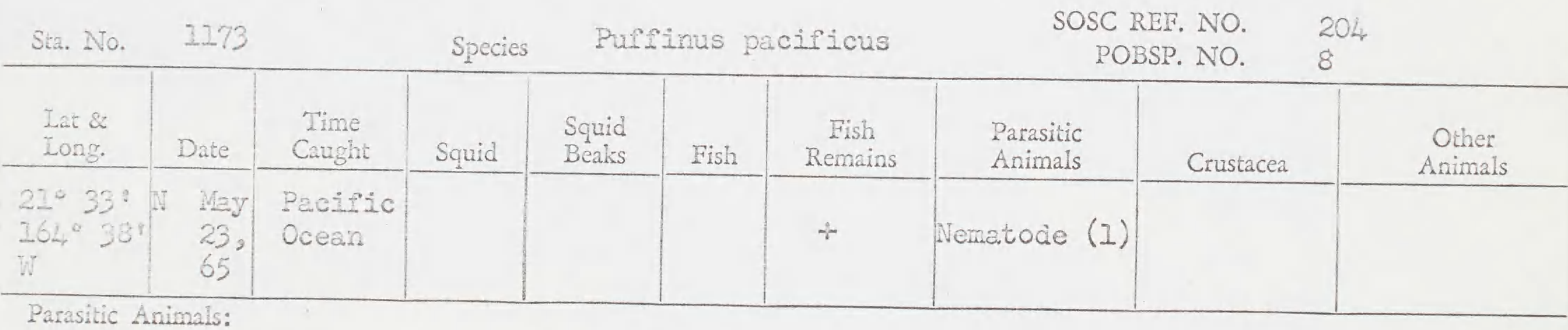

Parasitic Animals:

Notes on Squid:

lots: 2 fish remains nematode

Notes on Hish:

General Notes: 


\begin{tabular}{|c|c|c|c|c|c|c|c|c|c|}
\hline Sta. No. & 11? & & Speci & & is a & & & $\begin{array}{l}\text { NO. } \\
\text { NO. }\end{array}$ & \\
\hline $\begin{array}{l}\text { Lat \& } \\
\text { Long. }\end{array}$ & Date & $\begin{array}{l}\text { Time } \\
\text { Caught }\end{array}$ & Squid & $\begin{array}{l}\text { Squid } \\
\text { Beaks }\end{array}$ & Fish & $\begin{array}{c}\text { Fish } \\
\text { Remains }\end{array}$ & $\begin{array}{l}\text { Parasitic } \\
\text { Animals }\end{array}$ & Crustacea & $\begin{array}{c}\text { Other } \\
\text { Animals }\end{array}$ \\
\hline $\begin{array}{l}21^{\circ} 33 \cdot 1 \\
164^{\circ} 35^{\circ}\end{array}$ & $\begin{array}{l}-1 y \\
23, \\
65^{\circ}\end{array}$ & $\begin{array}{l}\text { Pacific } \\
\text { Ocean }\end{array}$ & & & & + & & & \\
\hline
\end{tabular}

Parasitic Animals:

Iots: I. fish remains

\section{Notes on Squid:}

Notes on Fist:

General Notes:

\section{PACIEIC OCEANOGRAPHIC BIOLOGICAL SURVEY PROGRAM}

SMITHSONIAN OCEANOGRAPHIC SORTING CENTER-SMITHSONIAN INSTITUTION

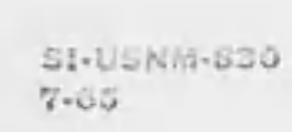

SOSC REF. NO. 204

\begin{tabular}{|c|c|c|c|c|c|c|c|c|c|}
\hline $\begin{array}{l}\text { Lat \& } \\
\text { Long. }\end{array}$ & Date & $\begin{array}{l}\text { Time } \\
\text { Caught }\end{array}$ & Squid & $\begin{array}{l}\text { Squid } \\
\text { Beaks }\end{array}$ & Fish & $\begin{array}{c}\text { Fish } \\
\text { Remains }\end{array}$ & $\begin{array}{l}\text { Parasitic } \\
\text { Animals }\end{array}$ & Crustacea & $\begin{array}{l}\text { Other } \\
\text { Animals }\end{array}$ \\
\hline $\begin{array}{l}21^{\circ} 34.1 \\
264^{\circ} \\
75\end{array}$ & $\begin{array}{l}\text { May } \\
23, \\
65\end{array}$ & $\begin{array}{l}\text { Pacific } \\
\text { Ocean }\end{array}$ & & & & - & & & \\
\hline
\end{tabular}

Parasitic Animals:

Lots: I digested remains

Notes on Squid:

Notes on Fish:

General Notes:

The remains are beyond identification.

PACIFIC OCEANOGRAPHIC BIOLOGICAL SURVEY PROGRAM

SMITHSONIAN OCEANOGRAPHIC SORTING CENTER—SMITHSONIAN INSTITUTION 
SOSC REF. NO. 204

St.. No. 1782

Species

Stema fuscata

POBSP. NO.

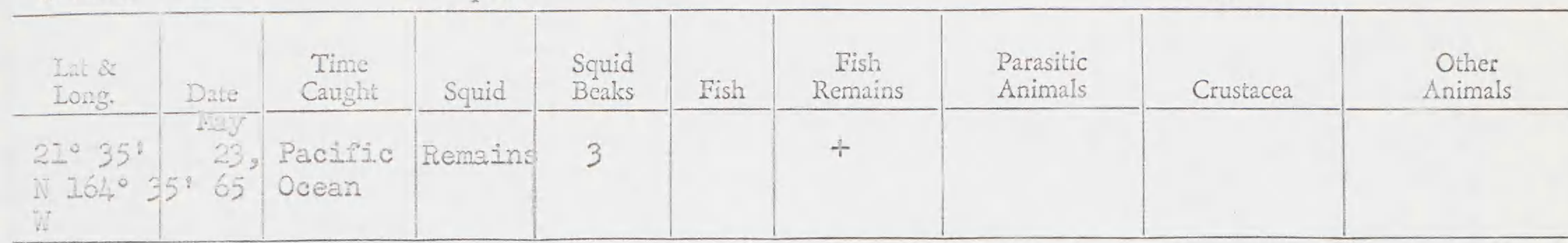

Parasitic Animals:

Lots: 3 fish remains

Notes on Squid:

squid beaks

suuid remains

Notes on Fish:

General Notes:

PACIFIC OCEANOGRAPHIC BIOLOGICAL SURVEY PROGRAM

SMITHSONIAN OCEANOGRAPHIC SORTING CENTER_-SMITHSONIAN INSTITUTION $2 / 28 / 66$

S6.USNM:G30

7.65

Skn. No. 1186

Species

Gygis a.lba.

SOSC REF. NO. 204 POBSP. NO. 8

\begin{tabular}{|c|c|c|c|c|c|c|c|c|c|}
\hline $\begin{array}{l}\text { Lat \& } \\
\text { Long. }\end{array}$ & Date & $\begin{array}{l}\text { Time } \\
\text { Caught }\end{array}$ & Squid & $\begin{array}{l}\text { Squid } \\
\text { Beaks }\end{array}$ & Fish & $\begin{array}{c}\text { Fish } \\
\text { Remains } \\
\end{array}$ & $\begin{array}{l}\text { Parasitic } \\
\text { Animals }\end{array}$ & Crustacea & $\begin{array}{c}\text { Other } \\
\text { Animals }\end{array}$ \\
\hline $\begin{array}{l}21^{\circ} 38^{\prime} \mathrm{N} \\
164^{\circ} 06^{\circ} \mathrm{T}\end{array}$ & $\begin{array}{l}\text { Vay } \\
23 \\
65\end{array}$ & $\begin{array}{l}\text { Pacific } \\
\text { Ocean }\end{array}$ & 1 & & 17 & + & & & \\
\hline
\end{tabular}

Parasitic Animals:

lots: 4 fish

Notes on Squid: Squid had tentacles partialiy detached.

squid

fish remains

digested remains

Notes on Fish: ${ }_{2}$. Two Ilsh with heads aigested. tion.

General Notes:

The remains are beyond identification.

PACIFIC OCEANOGRAPHIC BIOLOGICAL SURVEY PROGRAM

SMITHSONIAN OCEANOGRAPHIC SORTING CENTER—SMITHSONIAN INSTITUTION

S. Willis 2/10/66 
SOSC REF. NO. 204

\begin{tabular}{|c|c|c|c|c|c|c|c|c|c|}
\hline $\begin{array}{l}\text { Lat \& } \\
\text { Ing. }\end{array}$ & Date & $\begin{array}{l}\text { Time } \\
\text { Caught }\end{array}$ & Squid & $\begin{array}{l}\text { Squid } \\
\text { Beaks }\end{array}$ & Fish & $\begin{array}{c}\text { Fish } \\
\text { Remains }\end{array}$ & $\begin{array}{l}\text { Parasitic } \\
\text { Animals }\end{array}$ & Crustacea & $\begin{array}{l}\text { Other } \\
\text { Animals }\end{array}$ \\
\hline $\begin{array}{l}21^{\circ} 42^{\circ} \mathrm{N} \\
-61^{\circ} 50^{\circ} \mathrm{W}\end{array}$ & $\begin{array}{l}\text { Nay } \\
24 \\
65\end{array}$ & $\begin{array}{l}\text { Pacific } \\
\text { Ocean }\end{array}$ & & & & - & & & $\begin{array}{c}\text { Halobates } \\
(5)\end{array}$ \\
\hline
\end{tabular}

Parasitic Animals:

lots: 2 halobates

Notes on Sqquid: digested re... ins

Notes on Fish:

General Notes: 1. One halobates is a larval form.

2. The digested remains consist of bits of halobates, feathers, and several bones.

PACIFIC OCEAINOGRAPHIC BIOLOGICAL SURVEY PROGRAM

SMITHISONIAN OCEANOGRAPHIC SORTING CENTER_-SMITHSONIAN INSTITUTION
S. WIIIIS 3/2/66

S!- UENM- 250

$7 \cdot 04$

\begin{tabular}{|c|c|c|}
\hline $\begin{array}{l}\text { Lat \& } \\
\text { Long. }\end{array}$ & Date & $\begin{array}{l}\text { Time } \\
\text { Caught }\end{array}$ \\
\hline $\begin{array}{l}21^{\circ} 4.240 \\
1610^{\circ} 40^{\circ}\end{array}$ & $\begin{array}{l}\text { May } \\
249 \\
65\end{array}$ & $\begin{array}{l}\text { Pacific } \\
\text { Ocean }\end{array}$ \\
\hline
\end{tabular}

Species Puffinus pactricus

SOSC REF. NO. 204 POBSP. NO. 8

\begin{tabular}{|c|c|c|c|c|c|c|}
\hline Squid & $\begin{array}{l}\text { Squid } \\
\text { Beaks } \\
\end{array}$ & Fish & $\begin{array}{c}\text { Fish } \\
\text { Remains }\end{array}$ & $\begin{array}{l}\text { Parasitic } \\
\text { Animals }\end{array}$ & Crustacea & $\begin{array}{l}\text { Other } \\
\text { Animals }\end{array}$ \\
\hline & 111 & & - & & & \\
\hline
\end{tabular}

Notes on Squid:

lots: 2 squid beaks

Notes on Fish: 


\begin{tabular}{|c|c|c|c|c|c|c|c|c|c|}
\hline Sta. No. & 178 & & Specio & Puf. & is $\mathrm{p}$ & icus & & $\begin{array}{ll}\text { No. } & 2 \\
\text { NO. } & 8 \\
\end{array}$ & \\
\hline $\begin{array}{l}\text { Lat \& } \\
\text { Long. }\end{array}$ & Date & $\begin{array}{l}\text { Time } \\
\text { Caught }\end{array}$ & Squid & $\begin{array}{l}\text { Sryuid } \\
\text { Beaks }\end{array}$ & Fish & $\begin{array}{c}\text { Fish } \\
\text { Remains }\end{array}$ & $\begin{array}{l}\text { Parasitic } \\
\text { Animals }\end{array}$ & Crustacea & $\begin{array}{l}\text { Other } \\
\text { Animals }\end{array}$ \\
\hline $\begin{array}{l}22^{\circ} 43^{\prime N} \\
261^{\circ} 22^{\prime} \\
W\end{array}$ & $\frac{\text { May }}{24} \frac{15}{65}$ & $\begin{array}{l}\text { Pacific } \\
\text { Ocean }\end{array}$ & & 57 & & + & & & \\
\hline
\end{tabular}

Lots: 2 fish remains

Notes on Squid: squid beaks

Notes on Fish:

Gencral Notes:

\section{PACIFIC OCEANOGRAPHIC BIOLOGICAL SURVEY PROGRAM}

SMITHSONIAN OCEANOGRAPHIC SORTING CENTER—SMITHSONIAN INSTITUTION

Si-USWh:030

$\operatorname{tin}$
SOSC REF. NO. 204 POBSP. NO. 8

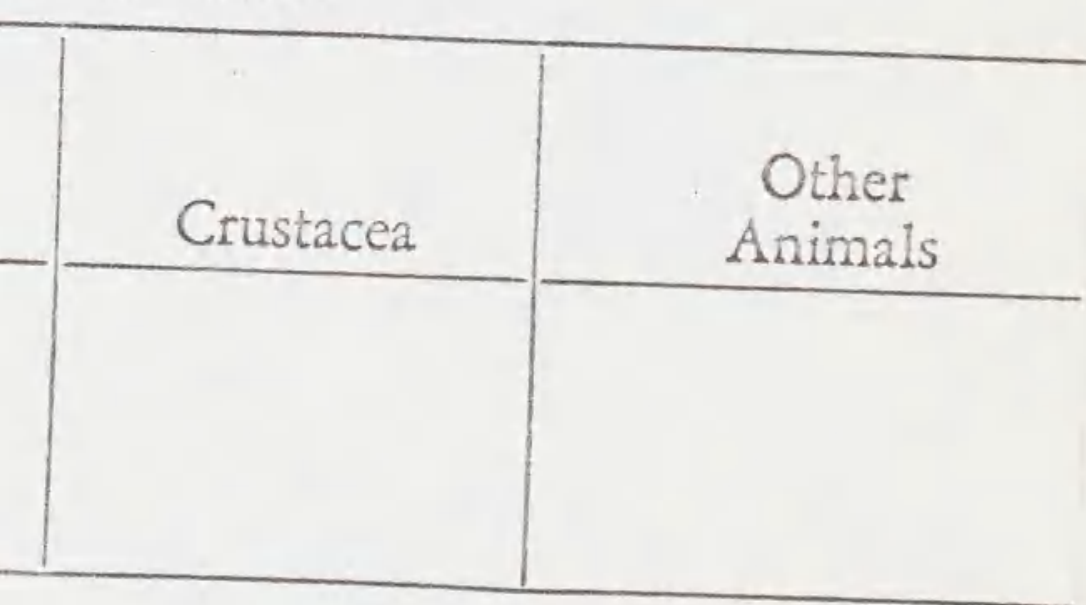

Iots: 2 squid beaks cebris

Notes on Fish:

Gencral Notes: 
Sta. No. 1292

\begin{tabular}{|c|c|}
\hline $\begin{array}{l}\text { Lat \& } \\
\text { Long. }\end{array}$ & Date \\
\hline $\begin{array}{l}21^{\circ} 43^{\circ} \mathrm{N} \\
161^{\circ} 27^{\circ} \\
\mathbb{N}\end{array}$ & $\begin{array}{l}\text { May } \\
242 \\
65\end{array}$ \\
\hline
\end{tabular}

Parasitic Animals:

Notes on Squid:

Notes on Fish:

General Notes:

The remains are beyond identification. Species Bulweria bulweris

lots: I digested remains

SOSC REF. NO. 204 POBSP. NO. 8

\begin{tabular}{|c|c|c|c|c|c|c|c|}
\hline $\begin{array}{l}\text { Time } \\
\text { Caught }\end{array}$ & Squid & $\begin{array}{l}\text { Squid } \\
\text { Beaks }\end{array}$ & Fish & $\begin{array}{c}\text { Fish } \\
\text { Remains }\end{array}$ & $\begin{array}{l}\text { Parasitic } \\
\text { Animals }\end{array}$ & Crustacea & $\begin{array}{l}\text { Other } \\
\text { Animals }\end{array}$ \\
\hline $\begin{array}{l}\text { Pacific } \\
\text { Ocean }\end{array}$ & & & & - & & & \\
\hline
\end{tabular}

\section{PACIEIC OCEANOGRAPHIC BIOLOGICAL SURVEY PROGRAM}

SMITHSONIAN OCEANOGRAPHIC SORTING CENTER—SMITHSONIAN INSTITUTION

S.6USNM.030
$\operatorname{los}$

S.0us
7.05
S. Willis $1 / 18 / 66$

\begin{tabular}{|c|c|c|c|c|c|c|c|c|c|}
\hline Sta. No. & 1192 & & Speci & Bu & eria & lweril & & $\begin{array}{l}\text { NO. } \\
\text { NO. }\end{array}$ & \\
\hline $\begin{array}{l}\text { Lat \& } \\
\text { Long. }\end{array}$ & Date & $\begin{array}{c}\text { Time } \\
\text { Caught }\end{array}$ & Squid & $\begin{array}{l}\text { Squid } \\
\text { Beaks } \\
\end{array}$ & Fish & $\begin{array}{c}\text { Fish } \\
\text { Remains }\end{array}$ & $\begin{array}{l}\text { Parasitic } \\
\text { Animals }\end{array}$ & Crustacea & $\begin{array}{l}\text { Other } \\
\text { Animals }\end{array}$ \\
\hline $\begin{array}{ll}22^{\circ} 44^{\circ} \mathrm{N} \\
262^{\circ}-13^{\circ} \mathrm{W}\end{array}$ & $\begin{array}{l}\text { Way } \\
24, \\
65\end{array}$ & $\begin{array}{l}\text { Pactilic } \\
\text { Ocean }\end{array}$ & & 4 & & + & & & \\
\hline
\end{tabular}

Notes on Squid:

lots: 2 fish remains squid beaks

Notes on Fish:

General Notes: 
SOSC REF. NO. 204

Stu. No. 2193

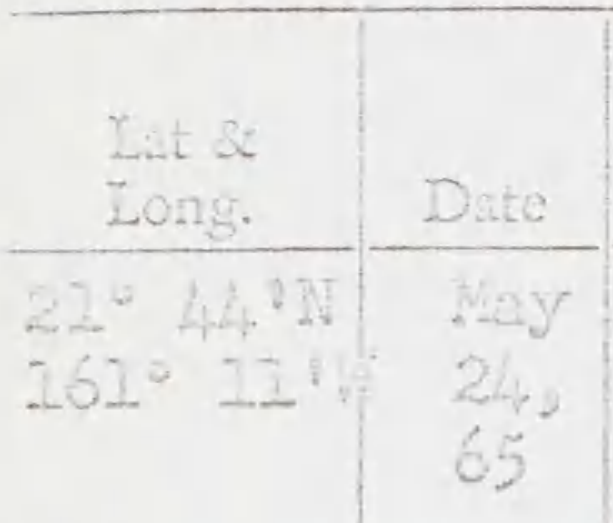

Parasitic Animals:

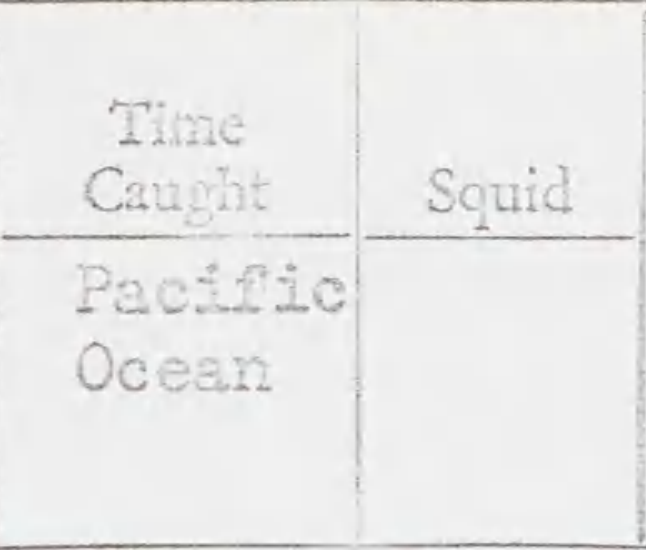

Species

Pufeinus pacieicus

POBSP. NO.

\begin{tabular}{|l|l|c|}
$\begin{array}{l}\text { Squid } \\
\text { Beaks }\end{array}$ & Fish & $\begin{array}{c}\text { Fish } \\
\text { Remains }\end{array}$ \\
\cline { 1 - 2 } 133 & & - \\
\hline
\end{tabular}

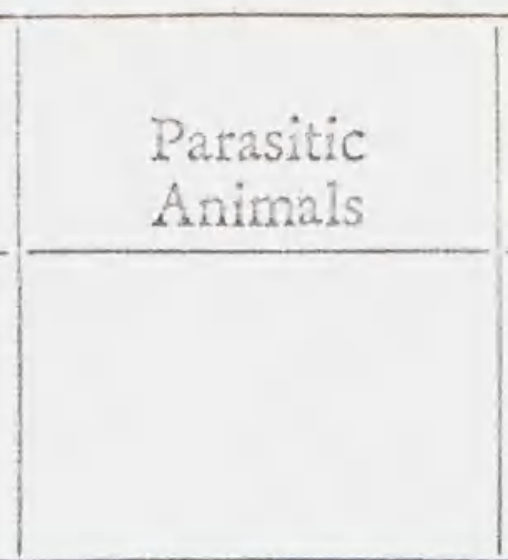

8

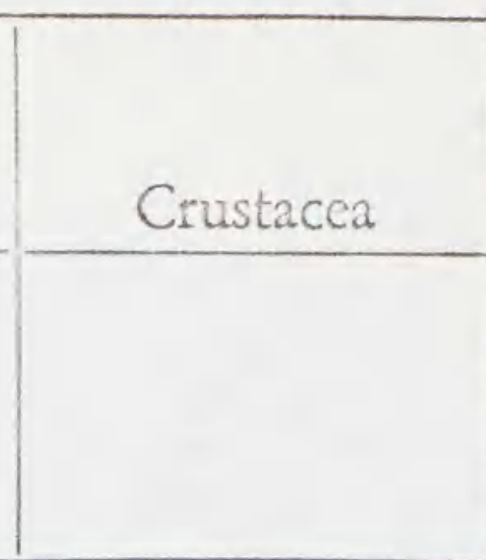

Other

Animals

lots: 2 squid beaks digested remains

Notes on Squid:

Notes on Fisin:

General Notes:

PACIEIC OCEANOGRAPHIC BIOLOGICAL SURVEY PROGRAM

SMITHSONIAN OCEANOGRAPHIC SORTING CENTER—SMITHSONIAN INSTITUTION

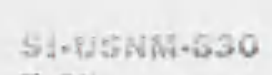

S. Willis $3 / 3 / 66$

7 -65

Sta. No. 2194

Species

Sterna fuscata

SOSC REF. NO. 204 POBSP. NO. 8

\begin{tabular}{|c|c|c|c|c|c|c|c|c|c|}
\hline $\begin{array}{l}\text { Lat \& } \\
\text { Long. }\end{array}$ & Date & $\begin{array}{l}\text { Time } \\
\text { Caught }\end{array}$ & Squid & $\begin{array}{l}\text { Squid } \\
\text { Beaks }\end{array}$ & Fish & $\begin{array}{c}\text { Fish } \\
\text { Remains }\end{array}$ & $\begin{array}{l}\text { Parasitic } \\
\text { Animals }\end{array}$ & Crustacea & $\begin{array}{l}\text { Other } \\
\text { Animals }\end{array}$ \\
\hline $\begin{array}{l}21^{\circ} 44 \mathrm{~N} \\
161^{\circ} 111^{\circ} \\
W\end{array}$ & $\begin{array}{l}\text { May } \\
24.2 \\
65\end{array}$ & $\begin{array}{l}\text { Pacific } \\
\text { Ocean }\end{array}$ & Remains & & & - & & & \\
\hline
\end{tabular}

Parasitic Animals:

Notes on Squid: Two squid beaks were left in the squid remains. Iots: I squid remains

Notes on Fish:

General Notes: 
SOSC REF. NO.

Sta. No. 229, Species Puffinus pacificus POBSP. NO.

\begin{tabular}{|c|c|c|c|c|c|c|c|c|c|}
\hline $\begin{array}{l}\text { Lit \& } \\
\text { Long. }\end{array}$ & Dite & $\begin{array}{l}\text { Tine } \\
\text { Can }\end{array}$ & Squid & $\begin{array}{l}\text { Squid } \\
\text { Beaks } \\
\end{array}$ & Fish & $\begin{array}{c}\text { Fish } \\
\text { Remains }\end{array}$ & $\begin{array}{l}\text { Parasitic } \\
\text { Animals }\end{array}$ & Crustaccá & $\begin{array}{l}\text { Other } \\
\text { Animals }\end{array}$ \\
\hline $\begin{array}{l}21^{\circ} 44^{\circ} \mathrm{N} \\
162^{\circ} \mathrm{11} \%\end{array}$ & $\begin{array}{l}\text { May } \\
24, \\
65\end{array}$ & $\begin{array}{l}\text { Pacific } \\
\text { Ocean }\end{array}$ & 1 & & & - & & & \\
\hline
\end{tabular}

Parasitic Animals:

lots: 2 squid

Notes on Squid: Squid has tentacles detached. digested remains

Notes on Fish:

General Notes:

Digested remains are beyond identification.

PACIFIC OCEANOGRAPHIC BIOLOGICAL SURVEY PROGRAM

SMITHSONIAN OCEANOGRAPHIC SORTING CENTER_-SMITHSONIAN INSTITUTION

S. Willis $3 / 3 / 66$

Sta. No. 2196

Species Sterna fuscata

SOSC REF. NO. 204

\begin{tabular}{|c|c|c|c|c|c|c|c|c|c|}
\hline $\begin{array}{l}\text { Lat \& } \\
\text { Long. }\end{array}$ & Date & $\begin{array}{l}\text { Time } \\
\text { Caught }\end{array}$ & Squid & $\begin{array}{l}\text { Squid } \\
\text { Beaks }\end{array}$ & Fish & $\begin{array}{c}\text { Fish } \\
\text { Remains }\end{array}$ & $\begin{array}{l}\text { Parasitic } \\
\text { Animals }\end{array}$ & Crustacea & $\begin{array}{l}\text { Other } \\
\text { Animals }\end{array}$ \\
\hline $\begin{array}{l}21^{\circ} 4{ }^{\circ} \\
162^{\circ} 11\end{array}$ & $\begin{array}{l}\text { May } \\
24 . \\
65\end{array}$ & $\begin{array}{l}\text { Pacific } \\
\text { Ocean }\end{array}$ & & & 1 & - & & & \\
\hline
\end{tabular}

Parasitic Animals:

lots: 2 fish

Notes on Squid:

Notes on Fish:

The fish is partially digested.

General Notes:

The remains are beyohd identification. 


\begin{tabular}{|c|c|c|c|c|c|c|c|c|c|}
\hline \multicolumn{3}{|c|}{2297} & Species & \multicolumn{3}{|c|}{ Bulweria bulwerif } & \multicolumn{3}{|c|}{$\begin{array}{c}\text { SOSC REF. NO. } 204 \\
\text { POBSP. NO. } 8\end{array}$} \\
\hline $\begin{array}{l}\text { Lat a } \\
\text { Long. }\end{array}$ & Date & $\begin{array}{l}\text { Time } \\
\text { Caught }\end{array}$ & Squid & $\begin{array}{l}\text { Squid } \\
\text { Beaks }\end{array}$ & Fish & $\begin{array}{c}\text { Fish } \\
\text { Remains }\end{array}$ & $\begin{array}{l}\text { Parasitic } \\
\text { Animals }\end{array}$ & Crustacea & $\begin{array}{l}\text { Other } \\
\text { Animals }\end{array}$ \\
\hline $\begin{array}{l}\left.22^{\circ} 42^{\circ}\right) \\
.62^{\circ} 010 \%\end{array}$ & $\begin{array}{l}\text { Hay } \\
240 \\
65\end{array}$ & $\begin{array}{l}\text { Pacilic } \\
\text { Ocean }\end{array}$ & $\begin{array}{r}\text { Eyes } \\
(5)\end{array}$ & & & + & & & \\
\hline
\end{tabular}

Parasitic Animals:

lots: 2 squid eyes

Notes on Squid: fish remains

Noces on Fish:

General Noles:

PACIFIC OCEANOGRAPHIC BIOLOGICAL SURVEY PROGRAM

SMITHSONIAN OCEANOGRAPHIIC SORTING CENTER_-SMITHSONIAN INSTITUTION

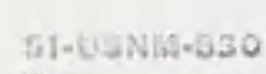

(2)

S. Wizlis $3 / 2 / 66$

SOSC REE. NO. 204

Sta. No. 1198

Species Sterna fuscata POBSP. NO. 8

\begin{tabular}{|c|c|c|c|c|c|c|c|c|c|}
\hline $\begin{array}{l}\text { Lat \&: } \\
\text { Long. }\end{array}$ & Date & $\begin{array}{l}\text { Time } \\
\text { Caught }\end{array}$ & Squid & $\begin{array}{l}\text { Squid } \\
\text { Beaks }\end{array}$ & Fish & $\begin{array}{c}\text { Fish } \\
\text { Remains }\end{array}$ & $\begin{array}{l}\text { Parasitic } \\
\text { Animals } \\
\end{array}$ & Crustacea & $\begin{array}{l}\text { Other } \\
\text { Animals }\end{array}$ \\
\hline $\begin{array}{l}22^{\circ} 45^{\circ} \mathrm{N} \\
260^{\circ} 54^{\circ} \mathrm{W}\end{array}$ & $\begin{array}{l}\text { May } \\
24 \\
65\end{array}$ & $\begin{array}{l}\text { Pacific } \\
\text { Ocean }\end{array}$ & & & & - & & & \\
\hline
\end{tabular}

Parasitic Animals:

Iots: I digested remains

Notes on Squid:

Notes on Fish:

General Notes: 
SOSC REF. NO. 204

Sta. No. 1199

Species

Bulweria bulwerit

POBSP. NO.

8

\begin{tabular}{|c|c|c|c|c|c|c|c|c|c|}
\hline $\begin{array}{l}\text { Lat of } \\
\text { Long. }\end{array}$ & Date & $\begin{array}{l}\text { Time } \\
\text { Caught }\end{array}$ & Squid & $\begin{array}{l}\text { Squid } \\
\text { Beaks }\end{array}$ & Fish & $\begin{array}{c}\text { Fish } \\
\text { Remains } \\
\end{array}$ & $\begin{array}{l}\text { Parasitic } \\
\text { Animals }\end{array}$ & Crustacea & $\begin{array}{c}\text { Other } \\
\text { Animals }\end{array}$ \\
\hline $\begin{array}{l}21^{\circ} 450 \mathrm{~N} \\
160^{\circ} 54^{\circ} \\
W\end{array}$ & $\begin{array}{l}112 y \\
24 \\
65\end{array}$ & $\begin{array}{l}\text { Pacific } \\
\text { Ocean }\end{array}$ & & & & - & & & \\
\hline
\end{tabular}

Parasitic Animals:

lots: 1 digested remains

Notes on Squid:

Notes on Fish:

General Notes:

The remains are beyond identification.

PACIEIC OCEANOGRAPHIC BIOLOGICAL SURVEY PROGRAM

SMITHSONIAN OCEANOGRAPHIC SORTING CENTER—SMITHSONIAN INSTITUTION

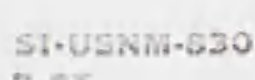

7.05

S. Willis $2 / 28 / 66$

SOSC REF. NO. 204

Sta. No. 1200

Species

Sterna fuscata

POBSP. NO. \&

\begin{tabular}{|c|c|c|c|c|c|c|c|c|c|}
\hline $\begin{array}{l}\text { Lat \& } \\
\text { Long. }\end{array}$ & Date & $\begin{array}{l}\text { Time } \\
\text { Caught }\end{array}$ & Squid & $\begin{array}{l}\text { Squid } \\
\text { Beaks }\end{array}$ & Fish & $\begin{array}{c}\text { Fish } \\
\text { Remains }\end{array}$ & $\begin{array}{l}\text { Parasitic } \\
\text { Animals }\end{array}$ & Crustacea & $\begin{array}{l}\text { Other } \\
\text { Animals }\end{array}$ \\
\hline $\begin{array}{r}22^{\circ} 45^{\circ} \mathrm{N} \\
160^{\circ} \\
48^{\circ} \mathrm{w}\end{array}$ & $\begin{array}{l}\text { May } \\
\frac{21}{65}\end{array}$ & $\begin{array}{l}\text { Pactific } \\
\text { Ocean }\end{array}$ & & & & -. & & & \\
\hline
\end{tabular}

Parasitic Animals:

Iots: 0

Notes on Squid:

Notes on Fish:

General Notes:

The stomach was empty.

PACIFIC OCEANOGRAPHIC BIOLOGICAL SURVEY PROGRAM

SMITHSONIAN OCEANOGRAPHIC SORTING CENTER_-SMITHSONIAN INSTITUTION 


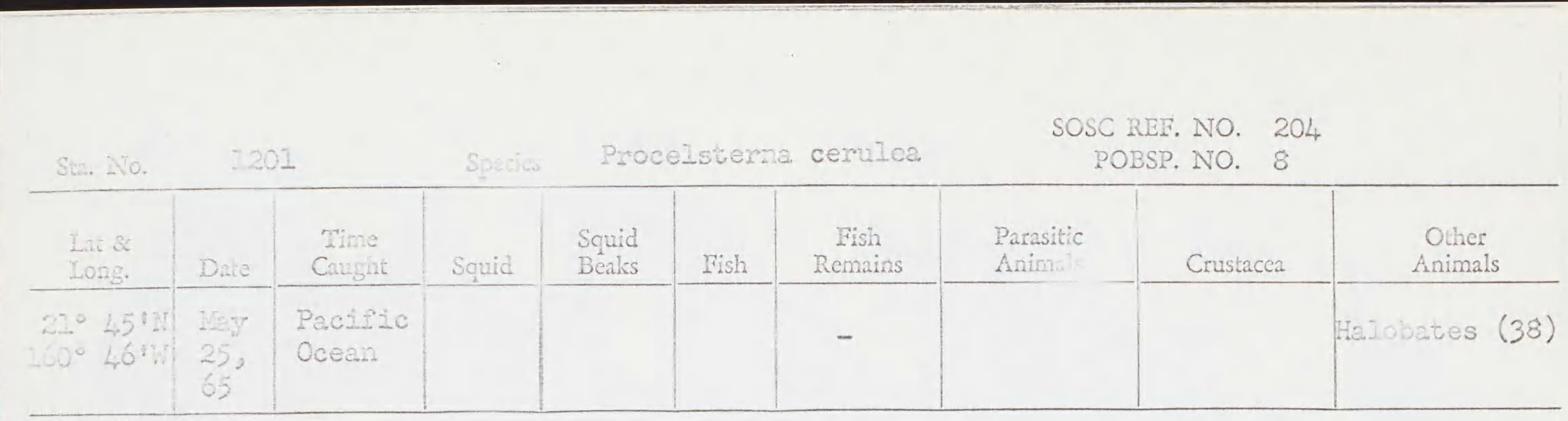

Parasitic Animals:

Iots: 2 hajobates

Notes on Squic:

halobates remalns

Motes on Fish:

General Notes:

PACIFIC OCEANOGRAPHIC BIOLOGICAL SURVEY PROGRAM

SMITHSO NIAN OCEANOGRAPHIC SORTING CENTER-SMITHSONIAN INSTITUTION

S. Wilizis $2 / 21 / 66$

ST-UENM-630

F+as

\begin{tabular}{|c|c|c|c|c|c|c|c|c|c|}
\hline Sta. No. & 1202 & & Speci & & Pinus & acificus & & $\begin{array}{l}\text { NO. } \\
\text { NO. }\end{array}$ & \\
\hline $\begin{array}{l}\text { Lat \& } \\
\text { Long. }\end{array}$ & Date & $\begin{array}{l}\text { Time } \\
\text { Caught }\end{array}$ & Squid & $\begin{array}{l}\text { Squid } \\
\text { Beaks } \\
\end{array}$ & Fish & $\begin{array}{c}\text { Fish } \\
\text { Remains }\end{array}$ & $\begin{array}{l}\text { Parasitic } \\
\text { Animals }\end{array}$ & Crustacea & $\begin{array}{l}\text { Other } \\
\text { Animals }\end{array}$ \\
\hline $\begin{array}{l}21^{\circ} 45^{4} N \\
160^{\circ} 46^{\circ} \\
N\end{array}$ & $\begin{array}{l}\text { May } \\
2 ! ; \\
65\end{array}$ & $\begin{array}{l}\text { Pacific } \\
\text { Ocean }\end{array}$ & & 32 & & - & & & \\
\hline
\end{tabular}

Parasitic Animals:

Lots: 2 digested remains

Nowe va Squid:

squid beaks

Notes on Bish:

General Notes: 


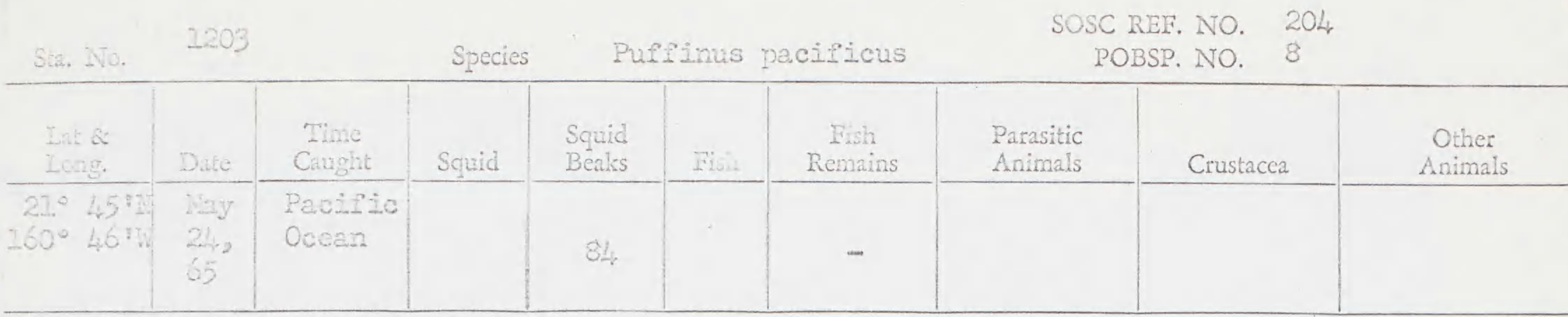

Parasitic Animals:

Iots: 2 squid beaks

Notes on Squid: digested remains

Notes on Fish:

Gonem. -Notes:

The remains are beyond identification.

PACIFIC OCEANOGRAPHIC BIOLOGICAL SURVEY PROGRAM

SMITHSONIAN OCEANOGRAPHIC SORTING CENTER_-SMITHSONIAN INSTITUTION

S. Ni12is $12 / 14 / 65$

S!.USNล1.030

Sta. No. 2204

Species

Puffinus paciricus

\begin{tabular}{|c|c|c|c|c|}
\hline $\begin{array}{l}\text { Time } \\
\text { Caught }\end{array}$ & Squid & $\begin{array}{l}\text { Squid } \\
\text { Beaks }\end{array}$ & Fish & $\begin{array}{l}\text { Fish } \\
\text { Remains }\end{array}$ \\
\hline $\begin{array}{l}\text { Pacific } \\
\text { Ocean }\end{array}$ & & 42 & & - \\
\hline
\end{tabular}

Parasitic Animals:

Notes on Squid:

SOSC REF. NO. POBSP. NO.

\begin{tabular}{|c|c|c|}
\hline $\begin{array}{l}\text { Parasitic } \\
\text { Animals }\end{array}$ & Crustacea & $\begin{array}{l}\text { Other } \\
\text { Animals }\end{array}$ \\
\hline
\end{tabular}

Lots: 2 digested remains squid beaks

Notes on Fish:

General Notes:

The romains we beyond identification. 


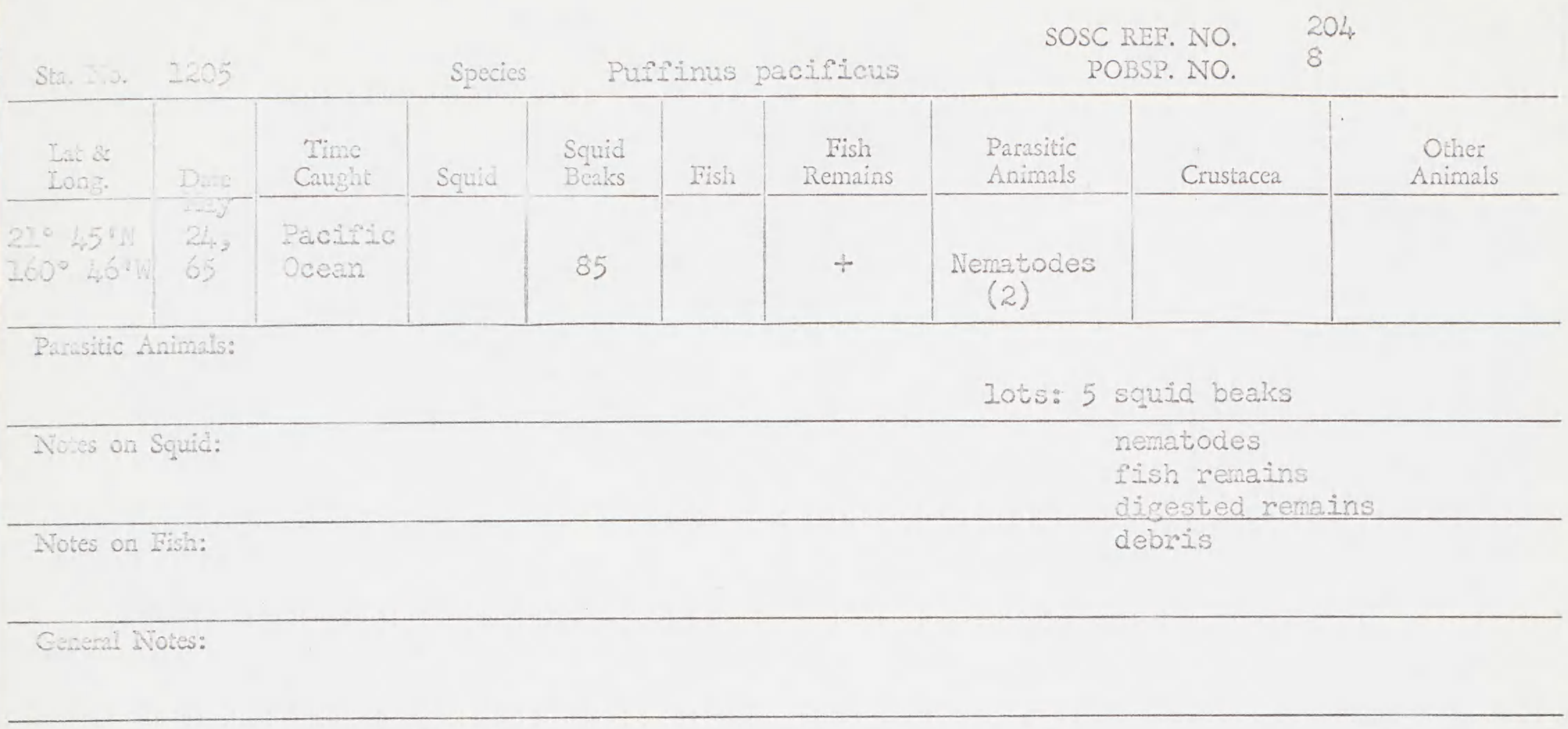

PACIFIC OCEANOGRAPHIC BIOLOGICAL SURVEY PROGRAM

SMITHSONIAN OCEANOGRAPHIC SORTING CENTER -SMITHSONIAN INSTITUTION

S. Willis $12 / 8 / 65$

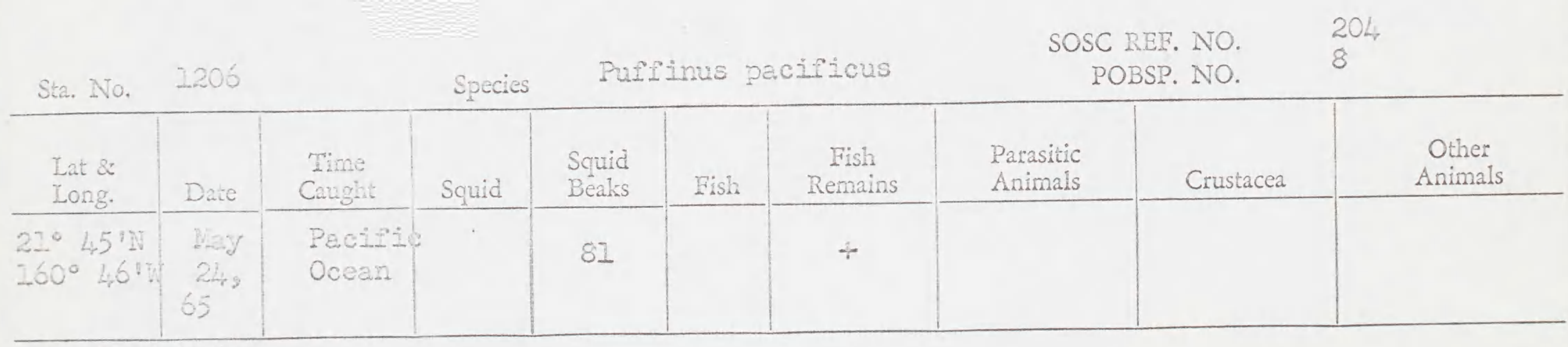

Parasitic Animals:

Lots: 3 squid beaks
digested remains
fish remains

Notes on Fish:

Notes:

PACIFIC OCEANOGRAPHIC BIOLOGICAL SURVEY PROGRAM 


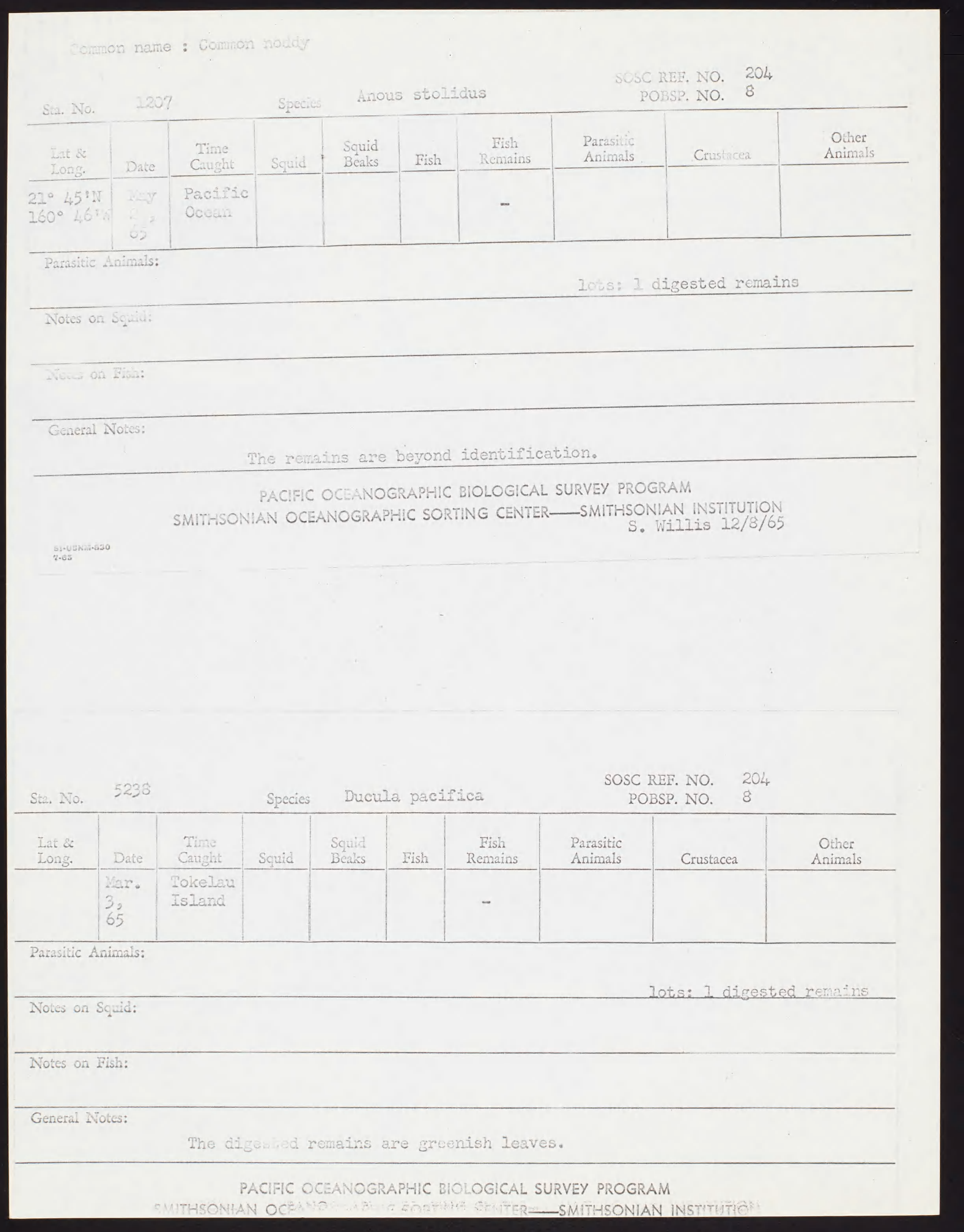




\begin{tabular}{|c|c|c|c|c|c|c|c|c|c|}
\hline Sta. No. & 5239 & & Species & Duci & pac & ica & & & \\
\hline \multirow[t]{2}{*}{$\begin{array}{l}\text { Lat \& } \\
\text { Long. }\end{array}$} & Date & $\begin{array}{l}\text { Tine } \\
\text { Caught }\end{array}$ & Squid & $\begin{array}{l}\text { Squid } \\
\text { Beaks } \\
\end{array}$ & Fish & $\begin{array}{c}\text { Fish } \\
\text { Remains }\end{array}$ & $\begin{array}{l}\text { Parasitic } \\
\text { Animals }\end{array}$ & Crustacea & $\begin{array}{l}\text { Ocher } \\
\text { Arimals }\end{array}$ \\
\hline & $\begin{array}{l}\text { Man. } \\
3, \\
65\end{array}$ & $\begin{array}{l}\text { Tokelau } \\
\text { Island }\end{array}$ & & & & - & & & \\
\hline
\end{tabular}

Parasitic Animals:

Iots: 1 digested remains

Notes on Squid:

Notes on lisite

Generni Notes:

The remains are bits of greenish leaves.

PACIFIC OCEANOGRAPHIC BIOLOGICAL SURVEY PROGRAM

SMITHSONIAN OCEANOGRAPHIC SORTING CENTER_-SMITHSONIAN INSTITUTION
S. WIIIIS $2 / 7 / 66$

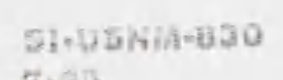

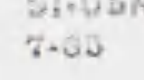

\begin{tabular}{|c|c|c|c|c|c|c|c|c|c|}
\hline Sta. No. & 5240 & & Specie & Ducu & pac: & & & $\begin{array}{ll}\text { No. } & 2 \\
\text { No. } & 8\end{array}$ & \\
\hline \multirow[t]{2}{*}{$\begin{array}{l}\text { Iat \&x } \\
\text { Long. }\end{array}$} & Date & $\begin{array}{l}\text { Time } \\
\text { Caught } \\
\end{array}$ & Squid & $\begin{array}{l}\text { Squid } \\
\text { Beaks }\end{array}$ & Fish & $\begin{array}{c}\text { Fish } \\
\text { Remains }\end{array}$ & $\begin{array}{l}\text { Parasitic } \\
\text { Animals }\end{array}$ & Crustacea & $\begin{array}{l}\text { Other } \\
\text { Animals }\end{array}$ \\
\hline & $\begin{array}{l}\text { Mar. } \\
32 \\
65\end{array}$ & $\begin{array}{l}\text { Iokelau } \\
\text { Island }\end{array}$ & & & & - & & & \\
\hline
\end{tabular}

Parasitic Arimals:

Iots: 2 digested remains

Notes on Squid:

Notes on Fish:

General Notes:

The debris is probably sone type of nut. It is shaped something like a Thantwo PIOLOGICAL SURVEY PROGRAM 
SOSC REF. NO. 204

Sta. No. 20262 Species

Sterna fuscata POBSP. NO.

8

\begin{tabular}{l|l|l|l|}
\hline Lat $\alpha$ & & & \\
Long. & Duts & & Squid \\
\hline & lug & Green & \\
& 6, & Isiand & \\
64 & & \\
\hline
\end{tabular}

\begin{tabular}{|c|c|c|}
$\begin{array}{c}\text { Squid } \\
\text { Beaks }\end{array}$ & Fish & $\begin{array}{c}\text { Fish } \\
\text { Remains }\end{array}$ \\
\hline & & - \\
& & \\
& &
\end{tabular}

\begin{tabular}{l} 
Parasitic \\
Animals \\
\hline \\
\hline
\end{tabular}

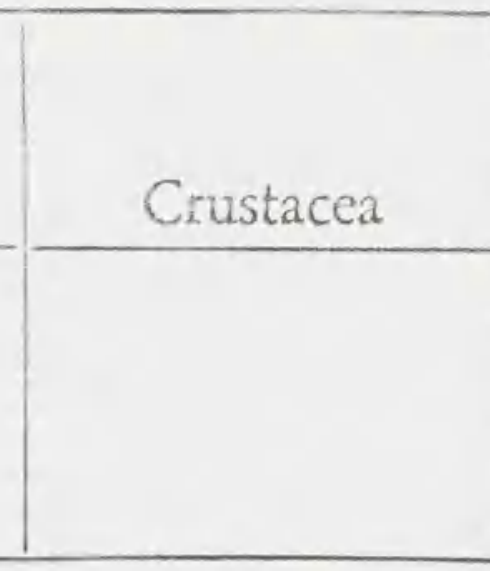

Parasitic Animals:

lots: 1 digested remains

Notes on Scuid:

Notes on Fish:

General Notes:

The remains are beyond identification.

PACIFIC OCEANOGRAPHIC BIOLOGICAL SURVEY PROGRAM

SMITHSONIAN OCEANOGRAPHIC SORTING CENTER—SMITHSONIAN INSTITUTION

Si-ushin-030

7.65

S. Willis $1 / 4 / 66$

\begin{tabular}{|c|c|c|c|c|c|c|c|c|c|}
\hline Sta. No. & 20274 & & Speci & & ma & cata & & $\begin{array}{l}\text { NO. } \\
\text { NO. }\end{array}$ & \\
\hline \multirow[t]{2}{*}{$\begin{array}{l}\text { Lat \& } \\
\text { Long. }\end{array}$} & Date & $\begin{array}{l}\text { Time } \\
\text { Caught }\end{array}$ & Squid & $\begin{array}{l}\text { Squid } \\
\text { Beaks }\end{array}$ & Fish & $\begin{array}{c}\text { Fish } \\
\text { Remains }\end{array}$ & $\begin{array}{l}\text { Parasitic } \\
\text { Animals }\end{array}$ & Crustacea & $\begin{array}{l}\text { Other } \\
\text { Animals }\end{array}$ \\
\hline & $\begin{array}{l}\text { Aug. } \\
18 \\
64\end{array}$ & $\begin{array}{l}\text { Green } \\
\text { Island }\end{array}$ & & & & - & & & \\
\hline
\end{tabular}

lots: 0

Notes on Squid:

Notes on Fish:

General Notes:

The stomach was empty.

PACIFIC OCEANOGRAPHIC BIOLOGICAL SURVEY PROGRAM

SMITHSONIAN OCEANOGRAPHIC SORTING CENTER—SMITHSONIAN INSTITUTION 
SOSC REF. NO. 204

Sta. No. 20279

Species

Sterna fuscata

POBSP. NO.

8

\begin{tabular}{|c|c|c|c|c|c|c|c|c|c|}
\hline $\begin{array}{l}\text { Lut \& } \\
\text { Long. }\end{array}$ & Date & $\begin{array}{l}\text { Time } \\
\text { Caught }\end{array}$ & Squid & $\begin{array}{l}\text { Squid } \\
\text { Bcaks }\end{array}$ & Fish & $\begin{array}{c}\text { Fish } \\
\text { Remains }\end{array}$ & $\begin{array}{l}\text { Parasitic } \\
\text { Animals }\end{array}$ & Crustacea & $\begin{array}{l}\text { Other } \\
\text { Animals }\end{array}$ \\
\hline & $\begin{array}{l}\text { Aus. } \\
20, \\
64\end{array}$ & $\begin{array}{l}\text { Green } \\
\text { Istand }\end{array}$ & & & & - & & & \\
\hline
\end{tabular}

Pansitic Animals:

Iots: I digested remains

Notes on Squid:

Notes on Fish:

General Notes:

The remains are beyond identification.

PACIFIC OCEANOGRAPHIC BIOLOGICAL SURVEY PROGRAM

SMITHSONIAN OCEANOGRAPHIC SORTING CENTER—SMITHSONIAN INSTITUTION

S. Willis 2/2I/66

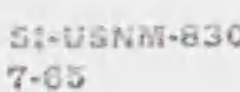

\begin{tabular}{|c|c|c|c|c|c|c|c|c|c|}
\hline Sta. No. & 20280 & & Specie & Ste: & fus & & & $\begin{array}{l}\text { NO. } \\
\text { NO. }\end{array}$ & \\
\hline \multirow[t]{2}{*}{$\begin{array}{l}\text { Lat \& } \\
\text { Long. }\end{array}$} & Date & $\begin{array}{l}\text { Time } \\
\text { Caught }\end{array}$ & Squid & $\begin{array}{l}\text { Squid } \\
\text { Beaks } \\
\end{array}$ & Fish & $\begin{array}{c}\text { Fish } \\
\text { Remains }\end{array}$ & $\begin{array}{l}\text { Parasitic } \\
\text { Animals }\end{array}$ & Crustacea & $\begin{array}{l}\text { Other } \\
\text { Animals }\end{array}$ \\
\hline & $\begin{array}{l}\text { Aug. } \\
28 \text {; } \\
64\end{array}$ & $\begin{array}{l}\text { Green } \\
\text { Island }\end{array}$ & & & & + & & & \\
\hline
\end{tabular}

Notes on Squid:

lots: 2 debris

fish remains

Notes on Fish:

The ish remains are bones and a few scales.

General Notes: 
SOSC REF. NO.

POBSP. NO.

Sta. No. 20284

Arenaria interpres

\begin{tabular}{|c|c|c|c|c|c|c|c|c|c|}
\hline $\begin{array}{l}\text { Lat \& } \\
\text { Long. }\end{array}$ & Date & $\begin{array}{l}\text { Time } \\
\text { Caughe }\end{array}$ & Squid & $\begin{array}{l}\text { Squid } \\
\text { Beaks }\end{array}$ & Fish & $\begin{array}{c}\text { Fish } \\
\text { Remains }\end{array}$ & $\begin{array}{l}\text { Parasitic } \\
\text { Animals }\end{array}$ & Crustacea & $\begin{array}{l}\text { Ot } \\
\text { Animais }\end{array}$ \\
\hline & $\begin{array}{l}\text { Sep. } \\
6 \\
64\end{array}$ & $\begin{array}{l}\text { Green } \\
\text { Island }\end{array}$ & & & & - & & & \\
\hline
\end{tabular}

Parasitic Animats:

lots: 2 insect remains

Notes on Squid: digested remans

Notes on Fish:

General Notes:

The insect remains are mostly heads.

PACIFIC OCEANOGRAPHIC BIOLOGICAL SURVEY PROGRAM

SMITHSONIAN OCEANOGRAPHIC SORTING CENTER—SMITHSONIAN INSTITUTION

S. Wi11is $12 / 8 / 65$

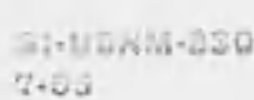

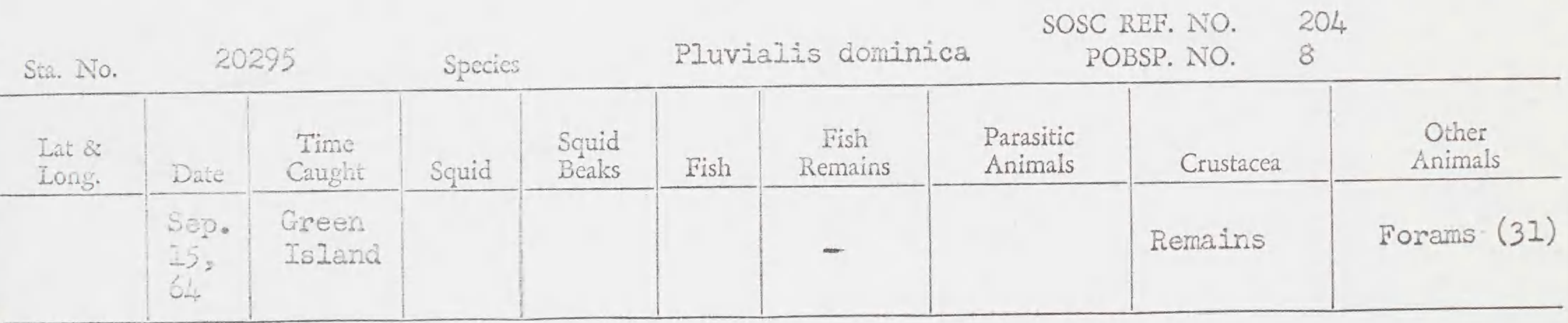

Parasitic Animals:

lots: 5

Notes on Squid:

forams

small stones

Notes on Fish:

debris

Gmeral Notes: I. Several claws wh oppendages are in the remains.

2. Numerous small stones were in the stomach contents.

P.CIFIC OCEANOGRAPHIC BIOLOGICAL SURVEY PROGRAM

SMITHSONIAN OCEANOGRAPHIC SORTING CENTER—SMITHSONIAN INSTITUTION 
SOSC RET. NO. $\quad 204$

Sta. No. 20309. Species Erolia melanotos

POBSP. NO.

8

\begin{tabular}{|c|c|c|c|c|c|c|c|c|c|}
\hline $\begin{array}{l}\text { Lat \& } \\
\text { Long. }\end{array}$ & Date & $\begin{array}{l}\text { Time } \\
\text { Caught }\end{array}$ & Squid & $\begin{array}{l}\text { Squid } \\
\text { Beaks }\end{array}$ & Fish & $\begin{array}{c}\text { Fish } \\
\text { Remains }\end{array}$ & $\begin{array}{l}\text { Parasitic } \\
\text { Animals }\end{array}$ & Crustacea & $\begin{array}{l}\text { Other } \\
\text { Animais }\end{array}$ \\
\hline & $\begin{array}{l}\text { Sep. } \\
25 \\
6 \%\end{array}$ & $\begin{array}{l}\text { Green } \\
\text { Island }\end{array}$ & & & & - & & & $\begin{array}{l}\text { Forams (7) } \\
\text { Insect } \\
\text { remains }\end{array}$ \\
\hline
\end{tabular}

Parasitic Animals:

lots: insect remains

Notes on Squid:

small. rocks and stones

Notes on Fish:

General Notes:

PACIFIC OCEANOGRAPHIC BIOLOGICAL SURVEY PROGRAM

SMITHSONIAN OCEANOGRAPHIC SORTING CENTER_SMITHSONIAN INSTITUTION S/WIIIIS $1 / 4 / 66$

5i-4)

$7 \cdot 65$

\begin{tabular}{|c|c|c|c|c|c|c|c|c|c|}
\hline Sta. No. & 2031 & & Species & Erol & mela & $\operatorname{tos}$ & & $\begin{array}{l}\text { F. NO. } 20 \\
\text { P. NO. } 8\end{array}$ & \\
\hline \multirow[t]{2}{*}{$\begin{array}{l}\text { Lat \& } \\
\text { Long. }\end{array}$} & Date & $\begin{array}{l}\text { Time } \\
\text { Caught }\end{array}$ & Squid & $\begin{array}{l}\text { Squid } \\
\text { Beaks }\end{array}$ & Fish & $\begin{array}{c}\text { Fish } \\
\text { Remains }\end{array}$ & $\begin{array}{l}\text { Parasitic } \\
\text { Animals }\end{array}$ & Crustacea & $\begin{array}{l}\text { Other } \\
\text { Animals }\end{array}$ \\
\hline & $\begin{array}{l}\text { Sep. } \\
25, \\
64\end{array}$ & $\begin{array}{l}\text { Green } \\
\text { Islana }\end{array}$ & & & & - & & Remains & Forams (12) \\
\hline
\end{tabular}

Parasitic Animals:

Notes on Squid:

Iots: 4 rocks (7)

crustacea remains

digested remains

Notes on Fish:

General Notes: 1. The rocks are mediturn sized.

2. The forams are pink colored. 


\begin{tabular}{|c|c|c|c|c|c|c|c|c|c|}
\hline Ste. No. & 203 & & Specie & & $011 a$ & lanotos & & $\begin{array}{l}\text { NO. } \\
\text { NO. }\end{array}$ & \\
\hline \multirow[t]{2}{*}{$\begin{array}{l}\text { Lat \& } \\
\text { Long. }\end{array}$} & Dite & $\begin{array}{l}\text { Time: } \\
\text { Cangite }\end{array}$ & Sequid & $\begin{array}{l}\text { Squid } \\
\text { Beaks }\end{array}$ & Fish & $\begin{array}{c}\text { Fish } \\
\text { Remains }\end{array}$ & $\begin{array}{l}\text { Parasitic } \\
\text { Animals }\end{array}$ & Crustacea & $\begin{array}{l}\text { Other } \\
\text { Animals }\end{array}$ \\
\hline & $\begin{array}{l}\text { Sep. } \\
25 \text {, }\end{array}$ & $\begin{array}{l}\text { Grian } \\
\text { Istand }\end{array}$ & & & & - & & & Forams (13) \\
\hline
\end{tabular}

Parasitic Animals:

lots: 3 forams

Notes on Squid: insect remoins rocks \& stones (22)

Noves on Fish:

General Notes:

The rocks and stones are of various sizes.

PACIFIC OCEANOGRAPHIC BIOLOGICAL SURVEY PROGRAM

SMITHSONIAN OCEANOGRAPHIC SORTING CENTER-SMITHSONIAN INSTITUTION S. Willis $1 / 4 / 66$

51-vexim-330

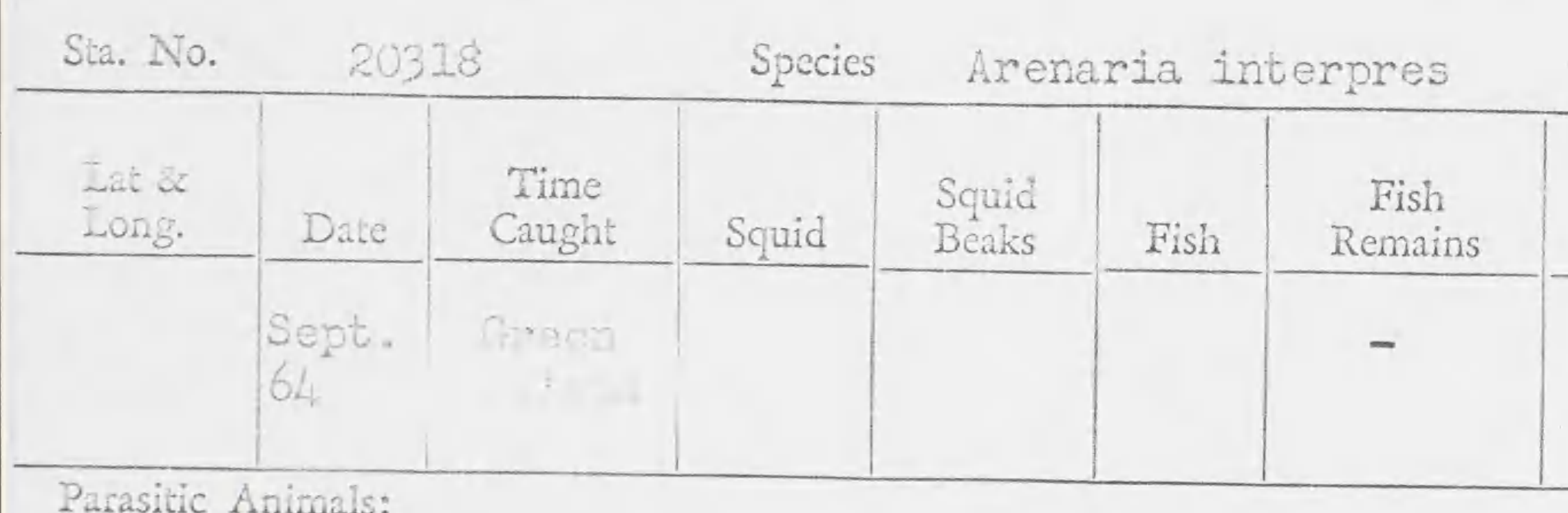

Parasitic Animals:

Notes on Squid:

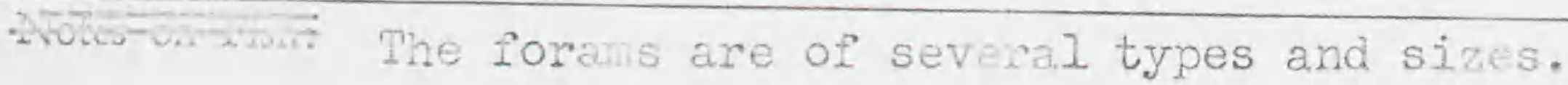

General Notes: The debris consist of numerous small rocks \& stones, insect particles.
SOSC REF. NO. 204 POBSP. NO.

\begin{tabular}{|c|c|c|c|}
$\begin{array}{c}\text { Parasitic } \\
\text { Animals }\end{array}$ & \multicolumn{1}{c}{$\begin{array}{c}\text { Other } \\
\text { Animals }\end{array}$} \\
\hline Crustacea & $\begin{array}{l}\text { Porams (56) } \\
\text { Gastropods (3) } \\
\text { Insect larva 1 }\end{array}$
\end{tabular}

lots: 4 foraminifera gastropods insect larva debris 
SOSC REF. NO. POBSP. NO.

\begin{tabular}{|c|c|c|c|c|c|c|c|c|c|}
\hline $\begin{array}{l}\text { Lat: \& } \\
\text { Long. }\end{array}$ & Date & $\begin{array}{l}\text { Time } \\
\text { Caught }\end{array}$ & Squid & $\begin{array}{l}\text { Squid } \\
\text { Bcaks }\end{array}$ & Fish & $\begin{array}{c}\text { Fish } \\
\text { Remains }\end{array}$ & $\begin{array}{l}\text { Parasitic } \\
\text { Animals }\end{array}$ & Crustacea & $\begin{array}{l}\text { Other } \\
\text { Animals }\end{array}$ \\
\hline & $\begin{array}{l}\text { Oot. } \\
2, \\
64\end{array}$ & $\begin{array}{l}\text { Green } \\
\text { Island }\end{array}$ & & & & - & & Remains & $\begin{array}{l}\text { Gastropod (1) } \\
\text { Forams (5I) }\end{array}$ \\
\hline
\end{tabular}

Parasitic Animals:

lots: 4 debris

Notes on Squid: gastropod

forams

crustacea remains

Notes on Fish:

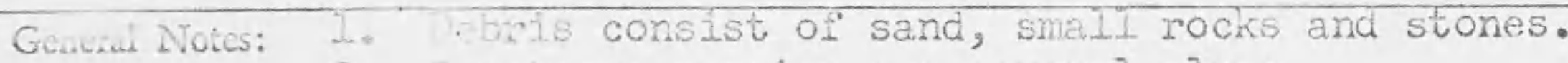

2. Custacea remains are several claws.

3. The forams are of various sizes and are similar to the drawing on the back.

PACIFIC OCEANOGRAPHIC BIOLOGICAL SURVEY PROGRAM

SMITHSONIAN OCEANOGRAPHIC SORTING CENTER—SMITHSONIAN INSTITUTION
5x-65inim-630
7.05
S. Willis $1 / 4 / 66$

\begin{tabular}{|c|c|c|c|c|c|c|c|c|c|}
\hline Sta. No. & 203 & & Specie: & $\mathrm{Plu}$ & is & inica & & $\begin{array}{l}\text { NO. } \\
\text { P. NO. }\end{array}$ & \\
\hline \multirow[t]{2}{*}{$\begin{array}{l}\text { Lat \& } \\
\text { Long. }\end{array}$} & Date & $\begin{array}{l}\text { Time } \\
\text { Caught }\end{array}$ & Squid & $\begin{array}{l}\text { Squid } \\
\text { Beaks } \\
\end{array}$ & Fish & $\begin{array}{c}\text { Fish } \\
\text { Remains }\end{array}$ & $\begin{array}{l}\text { Parasitic } \\
\text { Animals } \\
\end{array}$ & Crustacea & $\begin{array}{l}\text { Other } \\
\text { Animals }\end{array}$ \\
\hline & $\begin{array}{l}\text { Oct. } \\
9, \\
64\end{array}$ & $\begin{array}{l}\text { Green } \\
\text { Island }\end{array}$ & & & & - & & Remains & Forams (2) \\
\hline
\end{tabular}

Parasitic Animals:

lots: 4 crustacea remains

Notes on Squid:

insect remains

forams

debris

Notes on Fish:

General Notes:

The forams are the benthic type.

PACIFIC OCEANOGRAPHIC BIOLOGICAL SURVEY PROGRAM SMITHSONIAN OCEANOGRAPHIC SORTING CENTER_SMITHSONIAN INSTITUTION 
SOSC REF. NO.

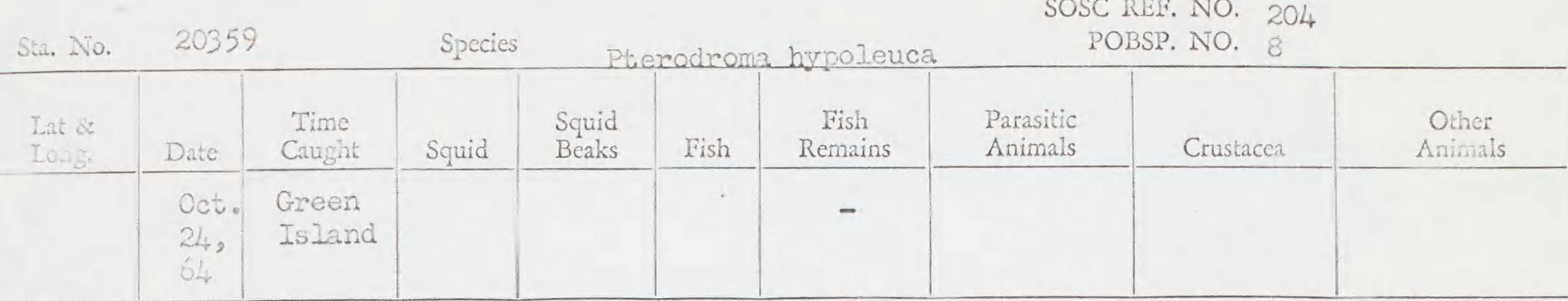

Purasitic Animals:

lots: 1 digested remains

Notes on Squid:

Notes on Fish:

General Notes:

The remains are beyond identification.

PACIFIC OCEANOGRAPHIC BIOLOGICAL SURVEY PROGRAM

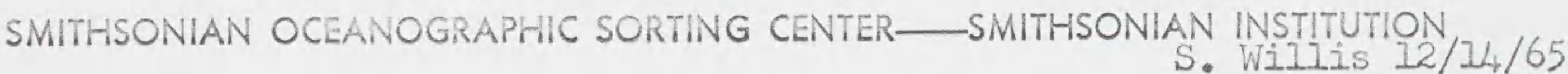

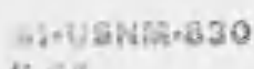

$\eta \cdot 65$

\begin{tabular}{|c|c|c|c|c|c|c|c|c|c|}
\hline Stâ. No. & 203 & & Speci & Su & leuc & aster & & $\begin{array}{l}\text { NO. } \\
\text { NO. }\end{array}$ & \\
\hline \multirow[t]{2}{*}{$\begin{array}{l}\text { Lat \& } \\
\text { Long. }\end{array}$} & Date & $\begin{array}{l}\text { Time } \\
\text { Caught }\end{array}$ & Squid & $\begin{array}{l}\text { Squid } \\
\text { Beaks }\end{array}$ & Fish & $\begin{array}{c}\text { Fish } \\
\text { Remains }\end{array}$ & $\begin{array}{l}\text { Parasitic } \\
\text { Animals }\end{array}$ & Crustacea & $\begin{array}{l}\text { Other } \\
\text { Animals }\end{array}$ \\
\hline & $\begin{array}{l}\text { Oct. } \\
30, \\
64\end{array}$ & $\begin{array}{l}\text { Green } \\
\text { Island }\end{array}$ & & & & - & & & \\
\hline
\end{tabular}

Parasitic Animals:

lots: I digested remains

Notes on Squid:

Notes on Fish:

Genend Notes:

One (I) squid beak was left in the remains.

PACIFIC OCEANOGRAPHIC BIOLOGICAI SURVEY PROGRAM

SMITHSONIAN OCEANOGRAPHIC SORTING CE 
SOSC REF. NO. $\quad 204$

S.a. No.

Species

Anous stolidus

POBSP. NO.
8

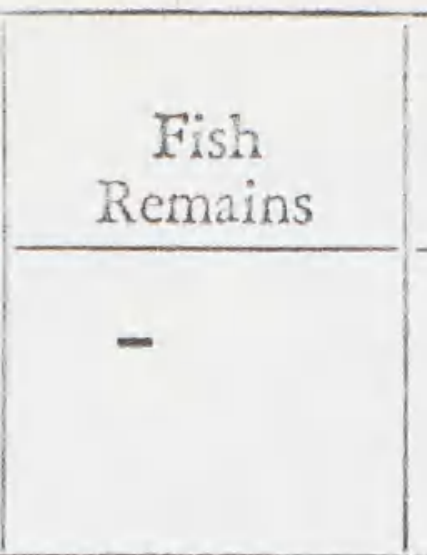

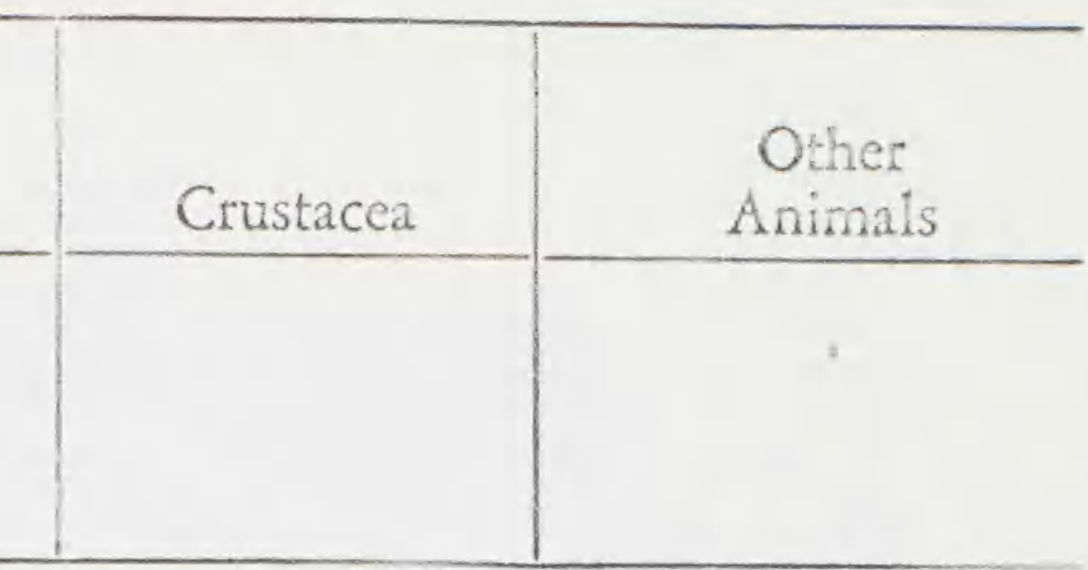

Parasitic Animals:

lots: 1 digested remains

Notes on Squid:

Notes on Fish:

General Notes:

The remains are beyond identification.

PACIFIC OCEANOGRAPHIC BIOLOGICAL SURVEY PROGRAM

SMITHSONIAN OCEANOGRAPHIC SORTING CENTER—SMITHSONIAN INSTITUTION 2/21/66

Si-CENh-630

$7+0 \bar{c}$

SOSC REF. NO. 204

POBSP. NO. 8

\begin{tabular}{|c|c|c|c|c|c|c|c|c|c|}
\hline $\begin{array}{l}\text { Lat \& } \\
\text { Long. }\end{array}$ & Date & $\begin{array}{l}\text { Time } \\
\text { Caught }\end{array}$ & Squid & $\begin{array}{l}\text { Squid } \\
\text { Beaks }\end{array}$ & Fish & $\begin{array}{c}\text { Fish } \\
\text { Remains }\end{array}$ & $\begin{array}{l}\text { Parasitic } \\
\text { Animals }\end{array}$ & Crustacea & $\begin{array}{l}\text { Other } \\
\text { Animals }\end{array}$ \\
\hline & $\begin{array}{l}\text { Nov. } \\
2 \\
64\end{array}$ & $\begin{array}{l}\text { Green } \\
\text { Island }\end{array}$ & & 24 & & $\begin{array}{l}\text { (59) eye- } \\
\text { balls }\end{array}$ & & & $\begin{array}{c}\text { Pelecypods } \\
(10)\end{array}$ \\
\hline
\end{tabular}

Parasitic Animals:

Iots: 4 squid beaks

eye balls

pelecypods

digested remains

Notes on Fish:

General Notes:

The digested remains consist of insect and other unidentifiable remains. 


\begin{tabular}{|c|c|c|c|c|c|c|c|c|c|}
\hline Sta. No. & 20375 & & Speci & & nar & interp & & $\begin{array}{l}\text { ․ NO. } \\
\text { No. }\end{array}$ & \\
\hline \multirow[t]{2}{*}{$\begin{array}{l}\text { Lat a } \\
\text { Long. }\end{array}$} & Date & $\begin{array}{l}\text { Tine } \\
\text { Caught }\end{array}$ & Squid & $\begin{array}{l}\text { Squid } \\
\text { Bcaks }\end{array}$ & Fish & $\begin{array}{c}\text { Fish } \\
\text { Remains }\end{array}$ & $\begin{array}{l}\text { Parasitic } \\
\text { Animals }\end{array}$ & Crustacea & $\begin{array}{l}\text { Other } \\
\text { Animals }\end{array}$ \\
\hline & $\begin{array}{l}\text { Nov. } \\
6 \% \\
64\end{array}$ & $\begin{array}{l}\text { Green } \\
\text { Island }\end{array}$ & & & & - & & Remains & $\begin{array}{l}\text { Ants (28) } \\
\text { Ant remains } \\
\text { Forams (9) }\end{array}$ \\
\hline
\end{tabular}

Parasitic Animals:

lots: 5 ints

Notes on Squid: ant rema.ins forams

crustacea remains

Notes on Fisti: rocks a stones

General Notes:

The ant remains consist of heads \& abdomens.

PACIFIC OCEANOGRAPHIC BIOLOGICAL SURVEY PROGRAM

SMITHSONIAN OCEANOGRAPHIC SORTING CENTER—SMITHSONIAN INSTITUTION

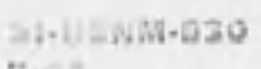

SOSC REF. NO. 204

Sta. No. 20379

Species

Anas acuta

POBSP. NO.

8

\begin{tabular}{|c|c|c|c|c|c|c|c|c|c|}
\hline $\begin{array}{l}\text { Lat \& } \\
\text { Long. }\end{array}$ & Dace & $\begin{array}{l}\text { Time } \\
\text { Caught }\end{array}$ & Squid & $\begin{array}{l}\text { Squid } \\
\text { Beaks }\end{array}$ & Fish & $\begin{array}{c}\text { Fish } \\
\text { Remains }\end{array}$ & $\begin{array}{l}\text { Parasitic } \\
\text { Animals }\end{array}$ & Crustacea & $\begin{array}{l}\text { Other } \\
\text { Animals }\end{array}$ \\
\hline & $\begin{array}{l}\text { Nov. } \\
7, \\
64\end{array}$ & $\begin{array}{l}\text { Green } \\
\text { Island }\end{array}$ & & & & - & & & $\begin{array}{r}\text { Foraminifera } \\
(14,1)\end{array}$ \\
\hline
\end{tabular}

Parasitic Animals:

lots: 2 foraminifera

Nores on Squid: debris

Notce on Fish:

General Notes:

1. Most of ho foraminifera have very hard shells.

2. The debris consist of numerous rocks and stones. 
Stat. No. 20380 Species

\begin{tabular}{|c|c|c|}
\hline Date & $\begin{array}{l}\text { Time } \\
\text { Caught }\end{array}$ & Squid \\
\hline $\begin{array}{l}\text { Nove } \\
\text { S3 } \\
6 l_{4}\end{array}$ & $\begin{array}{l}\text { Groen } \\
\text { Islend }\end{array}$ & \\
\hline
\end{tabular}

Parasitic Animals:

Notes on Squid:

Notes on Fish:

General Notes:
SOSC REF. NO. 204 POBSP. NO. 8

\begin{tabular}{|c|c|c|}
\hline $\begin{array}{l}\text { Squid } \\
\text { Bcaks }\end{array}$ & Fish & $\begin{array}{l}\text { Fish } \\
\text { Remains }\end{array}$ \\
\hline & & - \\
\hline
\end{tabular}

\begin{tabular}{l} 
Parasitic \\
Animals \\
\hline
\end{tabular}

Other Animals

Pelecypods

(2)

Iots: 3 pelecypods

insect remains

(1) medium stone

PACIFIC OCEANOGRAPHIC BIOLOGICAL SURVEY PROGRAM SMITHSONIAN OCEANOGRAPHIC SORTING CENTER—SMITHSONIAN INSTITUTION

SOSC REF. NO. 204 POBSP. NO. 8

\begin{tabular}{|c|c|c|c|c|c|c|c|c|c|}
\hline a. No. & 203 & & Specie & SuI & sula & & & NO. & \\
\hline $\begin{array}{l}\text { Lat \& } \\
\text { Long. }\end{array}$ & Date & $\begin{array}{l}\text { Time } \\
\text { Caught }\end{array}$ & Squid & $\begin{array}{l}\text { Squid } \\
\text { Beaks }\end{array}$ & Fish & $\begin{array}{c}\text { Fish } \\
\text { Remains }\end{array}$ & $\begin{array}{l}\text { Parasitic } \\
\text { Animals }\end{array}$ & Crustacea & $\begin{array}{l}\text { Other } \\
\text { Animals }\end{array}$ \\
\hline & $\begin{array}{l}\text { Nov. } \\
9_{2}\end{array}$ & $\begin{array}{l}\text { Green } \\
\text { Island }\end{array}$ & & 88 & & - & & & \\
\hline
\end{tabular}

Parasitic Animals:

lots: 3 digested remains

Notes on Squid: squid beaks debris

Notes on Fish:

General Notes:

The remains are beyond identification. 
SOSC REF. NO. 204

Sta. No. 20382

Species Larus species

POBSP. NO.

8

\begin{tabular}{|c|c|c|c|c|c|c|c|c|c|}
\hline Long. & Date & $\begin{array}{l}\text { Time } \\
\text { Caught }\end{array}$ & Squid & $\begin{array}{l}\text { Squid } \\
\text { Beaks }\end{array}$ & Fish & $\begin{array}{c}\text { Fish } \\
\text { Remains }\end{array}$ & $\begin{array}{l}\text { Parasitic } \\
\text { Animals }\end{array}$ & Crustacea & $\begin{array}{l}\text { Other } \\
\text { Animals }\end{array}$ \\
\hline & $\begin{array}{l}\text { Nov, } \\
20 \text {, } \\
64\end{array}$ & $\begin{array}{l}\text { Groen } \\
\text { Island }\end{array}$ & & & & + & & & \\
\hline
\end{tabular}

Putasitic Animals:

lots: I fish remains

Notes on Squid:

Notes on Fish:

General Notes:

PACIFIC OCEANOGRAPHIC BIOLOGICAL SURVEY PROGRAM

SMITHSONIAN OCEANOGRAPHIC SORTING CENTER_-SMITHSONIAN INSTITUTION

Si*USNM-

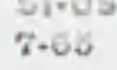

S. Willis $3 / 3 / 66$

\begin{tabular}{|c|c|c|c|c|c|c|c|c|c|}
\hline Sta. No. & & & Species & Mare & pen & & & $\begin{array}{l}\text { NO. } \\
\text { NO. }\end{array}$ & \\
\hline \multirow[t]{2}{*}{$\begin{array}{l}\text { Iat \& } \\
\text { Long. }\end{array}$} & Date & $\begin{array}{c}\text { Time } \\
\text { Caught }\end{array}$ & Squid & $\begin{array}{l}\text { Squid } \\
\text { Beaks } \\
\end{array}$ & Fish & $\begin{array}{c}\text { Fish } \\
\text { Remains }\end{array}$ & $\begin{array}{l}\text { Parasitic } \\
\text { Animals } \\
\end{array}$ & Crustacea & $\begin{array}{l}\text { Other } \\
\text { Animals }\end{array}$ \\
\hline & $\begin{array}{l}\text { Nov, } \\
20, \\
64\end{array}$ & $\begin{array}{l}\text { Green } \\
\text { Island }\end{array}$ & & & & - & $\begin{array}{c}\text { Nematodes } \\
\text { (8) }\end{array}$ & & \\
\hline
\end{tabular}

Notes on Squid:

Lots: 2. nematodes

debris

Notes on Fish:

General Notes:

The debris consist of nimerous rocks and stones.

PACIFIC OCEANOGRAPHIC BIOLOGICAL SURVEY PROGRAM

SMITHSONIAN OCEANOGRAPHIC SORTING CENTER—SMITHISONIAN INSTITUTION 
SOSC REE. NO. 204

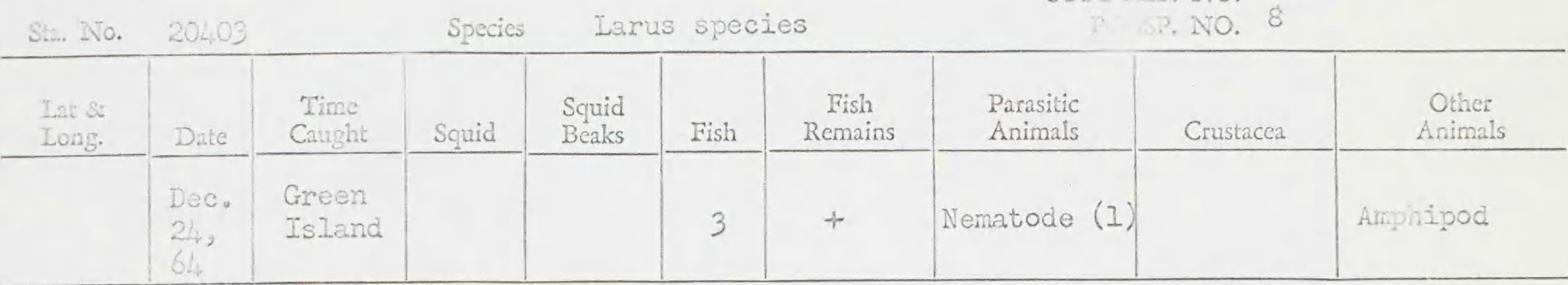

Parasitic Animals:

lots: 4 fish

Notes on Squid:

fish remains

nerlatode

amphipod

Notes on Fish: 1. Fish with heads digested.

2. Fish remains are bones except for two tails.

Guacral Notes:

PACIFIC OCEANOGRAPHIC BIOLOGICAL SURVEY PROGRAM

SMITHSONIAN OCEANOGRAPHIC SORTING CENTER—SMITHSONIAN INSTITUTION

S. Willis $1 / 18 / 66$

Di-LSWIR-650

$7 \cdot 60$

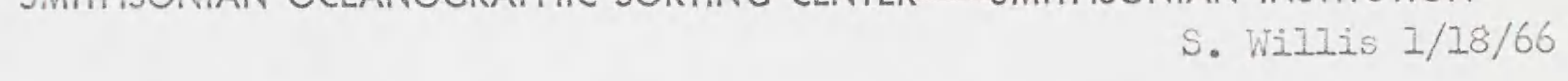

Sta. No. 20405

SOSC REF. NO. 204

\begin{tabular}{l|l|l|l|l|l|l|l|l} 
Lat \& \\
Long.
\end{tabular}

Parasitic Animals:

Iots: I digested remains

Notes on Squid:

Notes on Gish:

General Notes:

The djgenti remeins are greenish and beyond identification. 
Sta. No. 204Itr Species Larus species

\begin{tabular}{|c|c|c|c|c|c|c|c|c|c|}
\hline $\begin{array}{l}\text { Lat \& } \\
\text { Long. }\end{array}$ & Date & $\begin{array}{l}\text { Time } \\
\text { Caught }\end{array}$ & Squitis & $\begin{array}{l}\text { Squid } \\
\text { Beaks }\end{array}$ & Fish & $\begin{array}{c}\text { Fish } \\
\text { Remains }\end{array}$ & $\begin{array}{l}\text { Parasitic } \\
\text { Animals } \\
\end{array}$ & Crustacea & $\begin{array}{l}\text { Other } \\
\text { Animals }\end{array}$ \\
\hline & $\begin{array}{l}\text { Jan. } \\
\frac{17}{65}\end{array}$ & $\begin{array}{l}\text { Greon } \\
\text { Is land }\end{array}$ & & & 2 & + & & & \\
\hline
\end{tabular}

Parasitic Animals:

Notes on Sqquid:

Iots: 2 ish

fish remains

Notes on Fish:

The heads of both fish are digested.

General Notes:

PACIFIC OCEANOGRAPHIC BIOLOGICAL SURVEY PROGRAM

SMITHSONIAN OCEANOGRAPHIC SORTING CENTER-SMITHSONIAN INSTITUTION

S. Wilitis $3 / 3 / 66$

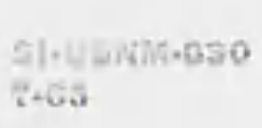

(1)

S

\begin{tabular}{|c|c|c|c|c|c|c|c|c|c|}
\hline Sta. No. & 20415 & & Species & Larus & argent & & & $\begin{array}{l}\text { NO. } \\
\text { NO. }\end{array}$ & \\
\hline \multirow[t]{2}{*}{$\begin{array}{l}\text { Lat \& } \\
\text { Long. }\end{array}$} & Date & $\begin{array}{l}\text { Time } \\
\text { Caught }\end{array}$ & Squid & $\begin{array}{l}\text { Squid } \\
\text { Beaks } \\
\end{array}$ & Fish & $\begin{array}{c}\text { Fish } \\
\text { Remains }\end{array}$ & $\begin{array}{l}\text { Parasitic } \\
\text { Animals }\end{array}$ & Crustacea & $\begin{array}{l}\text { Other } \\
\text { Animals }\end{array}$ \\
\hline & $\begin{array}{l}\text { Jan. } \\
20 \\
65\end{array}$ & $\begin{array}{l}\text { Greon } \\
\text { Island }\end{array}$ & Remaif & s 2 & & + & $\begin{array}{c}\text { Nematodes } \\
(4)\end{array}$ & & $\begin{array}{l}\text { Gastropod } \\
\text { (1) } \\
\text { Forams (62) }\end{array}$ \\
\hline
\end{tabular}

Notes on Squid:

lots: 9 squid remains squid beaks squid beak frag-

Notes on Fish: Fish remains are six bones. ments fish remains nernatodes gastropod

General Notes: 1. The forams are of various types and sizes. 2. Numerous wite rocks, stones, and bits of shell are forams digested remains in the debris. debris 
SOSC REF. NO. 204

POBSP. NO. 8

\begin{tabular}{|c|c|c|c|c|c|c|c|c|c|}
\hline $\begin{array}{l}\text { Lat \& } \\
\text { Loag. }\end{array}$ & Date & $\begin{array}{l}\text { Time } \\
\text { Caught }\end{array}$ & Squid & $\begin{array}{l}\text { Squid } \\
\text { Beaks }\end{array}$ & Fish & $\begin{array}{l}\text { Fish } \\
\text { Remains }\end{array}$ & $\begin{array}{l}\text { Parasitic } \\
\text { Animals }\end{array}$ & Crustacea & $\begin{array}{l}\text { Other } \\
\text { Animals }\end{array}$ \\
\hline & $\begin{array}{l}\text { Feb. } \\
26= \\
65\end{array}$ & $\begin{array}{l}\text { Green } \\
\text { Island }\end{array}$ & Remains & 25 & & - & & & \\
\hline
\end{tabular}

Parasitic Animals:

Iots: 3 squid beaks

Notes on Squid:

debols

remains

Notes on Fish:

General Notes:

The debris is possibly a lime rock.

PACIFIC OCEANOGRAPHIC BIOLOGICAL SURVEY PROGRAM

SMITHSONIAN OCEANOGRAPHIC SORTING CENTER—SMITHSONIAN INSTITUTION

S. Willis $1 / 10 / 66$

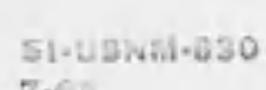

$7+60$

(n)

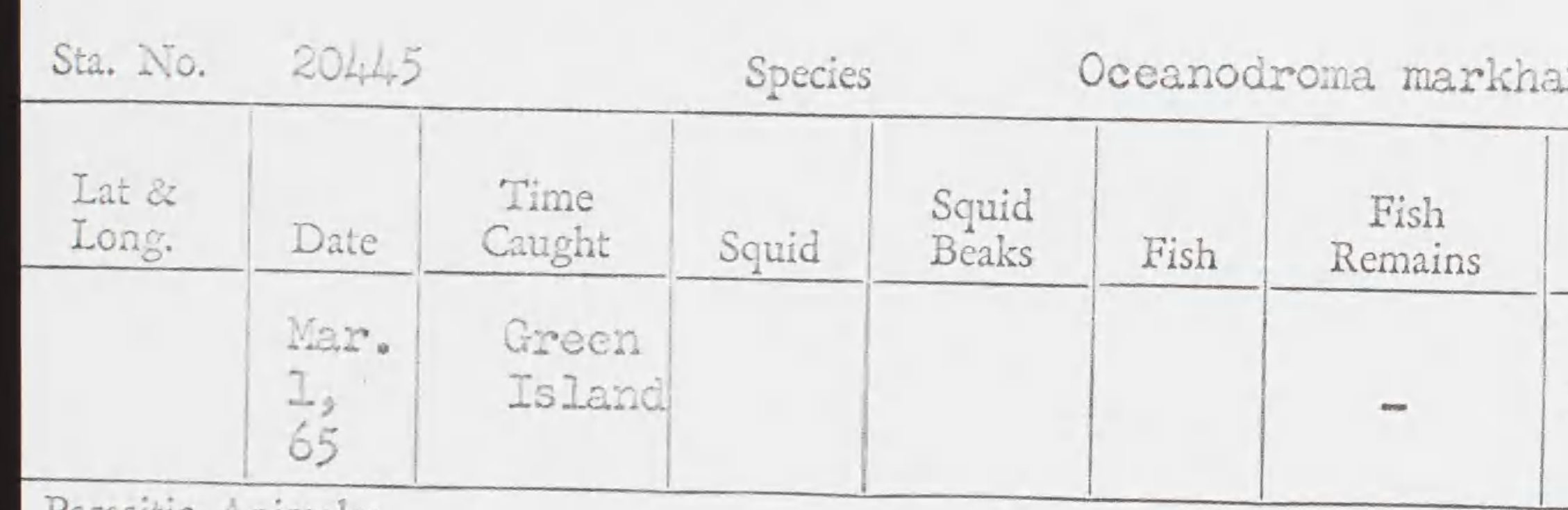

Parasitic Animals:

Notes on Squid:

Notes on Fish:

General Notes: ‥ The stones are mediun size an Mlotable.

2. The djgested remains contain one squid beak and feather. The other material is beyond identification. 
Sta. No. Specie

Fin 100 peregrinus

\begin{tabular}{l|l|l}
$\begin{array}{c}\text { Squid } \\
\text { Beaks }\end{array}$ & Fish & $\begin{array}{c}\text { Fish } \\
\text { Remains }\end{array}$ \\
\hline & -
\end{tabular}

SOSC REF. NO. 204 POBSP. NO.

\begin{tabular}{|l|l|c}
$\begin{array}{c}\text { Parasitic } \\
\text { Animals }\end{array}$ & Crustacea & $\begin{array}{c}\text { Other } \\
\text { Animals }\end{array}$ \\
\hline & & \\
&
\end{tabular}

Parasitic Animas:

Iots: I digested remains

Notes on Squid:

Notes on Fish:

General Notes:

Bits of feathers are in the digested remains.

PACIFIC OCEANOGRAPHIC BIOLOGICAL SURVEY PROGRAM

SMITHSONIAN OCEANOGRAPHIC SORTING CENTER-SMITHSONIAN INSTITUTION

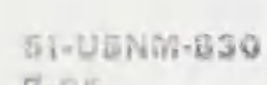

SOSC REF. NO. 204

Sta, No $2044 ?$ Species Lams snectes

\begin{tabular}{|l|l|l|l|l|}
\hline $\begin{array}{l}\text { Time } \\
\text { Carght }\end{array}$ & Squic & $\begin{array}{c}\text { Squid } \\
\text { Beaks }\end{array}$ & Fish & $\begin{array}{c}\text { Fish } \\
\text { Remains }\end{array}$ \\
\hline $\begin{array}{l}\text { Green } \\
\text { Island }\end{array}$ & & & & + \\
\hline
\end{tabular}

Parasitic Animals:

\begin{tabular}{|c|c|c}
$\begin{array}{c}\text { Parasitic } \\
\text { Animals }\end{array}$ & Crustacea & \multicolumn{1}{|c}{$\begin{array}{c}\text { Other } \\
\text { Animals }\end{array}$} \\
\cline { 1 - 2 } $\begin{array}{c}\text { Nematodes } \\
\text { (4) }\end{array}$ & & $\begin{array}{l}\text { Gastropod (41) } \\
\text { Forams (675) }\end{array}$ \\
\hline
\end{tabular}

lots: 5 fish remains

Notes on Squid:

Notes on Wish: 
SOSC REE. NO. 204 Species Larus occidentalis POBSP. NO. 8

\begin{tabular}{|c|c|c|c|c|c|c|c|c|c|}
\hline Inter & Date & $\begin{array}{l}\text { Time } \\
\text { Canght: }\end{array}$ & Squid & $\begin{array}{l}\text { Squid } \\
\text { Beaks }\end{array}$ & Fish & $\begin{array}{c}\text { Fish } \\
\text { Remains }\end{array}$ & $\begin{array}{l}\text { Parasitic } \\
\text { Animals }\end{array}$ & Crustacea & $\begin{array}{l}\text { Othe: } \\
\text { Animals }\end{array}$ \\
\hline & 65 & $\begin{array}{l}\text { Green } \\
\text { Istand }\end{array}$ & & & & - & & Remains & \\
\hline
\end{tabular}

Rarasitic Animals:

lots: 3 crustacea remains

Notes on Squid: algested remains debris

Notes on Fish:

General Notes: The debris is bits of shell.

PACIFIC OCEANOGRAPHIC BIOLOGICAL SURVEY PROGRAM SWITHSONIAN OCEANOGRAPHIC SORTING CENTER—SMITHSONIAN INSTITUTION

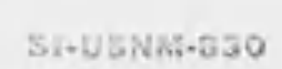
$7 \cdot 65$ S. Willis $1 / 25 / 66$

Sra. No. 204.49 Species

Larus species

\begin{tabular}{|c|c|c|c|c|c|c|}
\hline Date & $\begin{array}{l}\text { Time } \\
\text { Caught }\end{array}$ & Squid & $\begin{array}{l}\text { Squid } \\
\text { Beaks }\end{array}$ & Fish & $\begin{array}{c}\text { Fish } \\
\text { Remains }\end{array}$ & $\begin{array}{l}\text { Parasitic } \\
\text { Animals }\end{array}$ \\
\hline $\begin{array}{l}\text { Mar. } \\
\frac{9}{65}\end{array}$ & $\begin{array}{l}\text { Green } \\
\text { Island }\end{array}$ & & & & + & \\
\hline
\end{tabular}

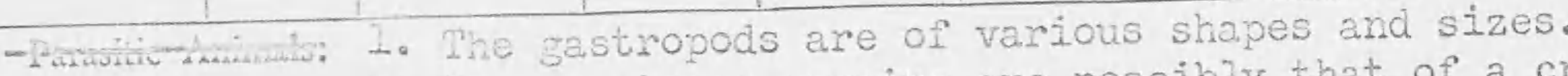
2. The crustacea remains are possibly that of a crab.
SOSC REF. NO. 204 POBSP. NO.

\begin{tabular}{|l|l} 
Crustacea & \multicolumn{1}{c}{$\begin{array}{c}\text { Other } \\
\text { Animals }\end{array}$} \\
\hline Remains & $\begin{array}{l}\text { Forans (576) } \\
\text { Pelecypod (1) } \\
\text { Gastropods } \\
100)\end{array}$ \\
\hline
\end{tabular}

lots: 7 forams
-Nutr Un=squth Numerous white rocks, stones, and bits of shell are in the debris. Also, possibly some unidentified forams. ish remains crustacea rem. digested remains pelecypoc gastropods debris

Notes on Fish: The fish remains are bones and scales.

to the diagram General Notes: I. The forams are of various sizes and types. They are similar to the

PACIFIC OCEANOGRAPHIC BIOLOGICAL SURVEY PROGRAM SMITHSONIAN OCEANOGRAPHIC SORTING CENTER_SMITHSONIAN INSTITUTION 
SOSC REF. NO. 204

Sta. No. $20450 \quad$ Species Larus species POBSP. NO.

\begin{tabular}{|c|c|c|c|c|c|c|c|c|c|}
\hline $\begin{array}{l}\text { Lat \& } \\
\text { Long. }\end{array}$ & Date & $\begin{array}{l}\text { Time } \\
\text { Caught }\end{array}$ & Squid & $\begin{array}{l}\text { Squid } \\
\text { Beaks }\end{array}$ & Fish & $\begin{array}{c}\text { Fish } \\
\text { Remains }\end{array}$ & $\begin{array}{l}\text { Parasitic } \\
\text { Animals }\end{array}$ & Crustacea & $\begin{array}{l}\text { Other } \\
\text { Animals }\end{array}$ \\
\hline & $\begin{array}{l}\text { Mar. } \\
9, \\
65\end{array}$ & $\begin{array}{l}\text { Green } \\
\text { IsIand }\end{array}$ & & & & + & $\begin{array}{l}\text { Nematode } \\
\text { (I) }\end{array}$ & Remains & Forams (16) \\
\hline
\end{tabular}

Parasitic Animals:

10ts: 5 fish remains

Notes on Squid:

Nematode

crustacea remains

forams

Notes on Fish: 1 fish tail and numerous bones in remains. debris

Several bits of rock and stone are intemingled with the bones.

General Notes: 1. Debris consist of bits of shell and small stones.

2. The remains (crustacea) contain several small claws.

PACIFIC OCEANOGRAPHIC BIOLOGICAL SURVEY PROGRAM

SMITHSONIAN OCEANOGRAPHIC SORTING CENTER_-SMITHSONIAN INSTITUTION

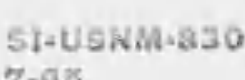

S. WIIIis $1 / 10 / 66$

$7 \cdot 0 x$

\begin{tabular}{|c|c|c|c|c|c|c|c|c|c|}
\hline Sta. No. & 20452 & & Species & Phas & on $x$ & icauda & & $\begin{array}{l}\text { No. } \\
\text { NO. }\end{array}$ & \\
\hline \multirow[t]{2}{*}{$\begin{array}{l}\text { Iat \&c } \\
\text { Long. }\end{array}$} & Date & $\begin{array}{l}\text { Time } \\
\text { Caught }\end{array}$ & Squid & $\begin{array}{l}\text { Squid } \\
\text { Beaks }\end{array}$ & Fish & $\begin{array}{c}\text { Fish } \\
\text { Remains }\end{array}$ & $\begin{array}{l}\text { Parasitic } \\
\text { Animals }\end{array}$ & Crustacea & $\begin{array}{l}\text { Other } \\
\text { Animals }\end{array}$ \\
\hline & $\begin{array}{l}\text { War. } \\
8, \\
65\end{array}$ & $\begin{array}{l}\text { Green } \\
\text { Island }\end{array}$ & Remaing & & 1 & + & & & \\
\hline
\end{tabular}

Parasitic Animals:

lots: 3 squid remains

Notes on Squid:

The equid remains are two groups of tentacles.

rish

fish remains

Notes on Fish:

General Notes: lio stomach was received. These contents are the products of recurgitation. 
Sta. No. 20453

\begin{tabular}{|c|c|c|c|c|c|c|}
\hline $\begin{array}{l}\text { Lat \& } \\
\text { Long. }\end{array}$ & Date & $\begin{array}{l}\text { Time } \\
\text { Caught } \\
\end{array}$ & Squid & $\begin{array}{l}\text { Squid } \\
\text { Beaks }\end{array}$ & Iish & $\begin{array}{l}\text { Fish } \\
\text { Remains }\end{array}$ \\
\hline & $\begin{array}{l}\text { Has. } \\
\frac{17}{65}\end{array}$ & $\begin{array}{l}\text { Green } \\
\text { Island }\end{array}$ & & & & - \\
\hline
\end{tabular}

Pacasitic AnimaIs:

Notes on Squid:

Notes on Fish:

General Notes: 1. The rocks are of various sizes.

2. The digested remains are greenish and beyond identification.

PACIFIC OCEANOGRAPHIC BIOLOGICAL SURVEY PROGRAM

SMITHSONIAN OCEANOGRAPHIC SORTING CENTER—SMITHSONIAN INSTITUTION

S. Willis $1 / 4 / 66$

lots: 2 rocks (83)
ธ1-บอง

7.65
SOSC REF. NO. 204 POBSP. NO.

SOSC REF. NO. 204

Sta. No. 20454

Anas acuta.

POBSP. NO. 8

\begin{tabular}{|c|c|c|c|c|c|c|c|c|c|}
\hline $\begin{array}{l}\text { Lat \& } \\
\text { Long. }\end{array}$ & Date & $\begin{array}{l}\text { Time } \\
\text { Caught }\end{array}$ & Squid & $\begin{array}{l}\text { Squid } \\
\text { Beaks } \\
\end{array}$ & Fish & $\begin{array}{c}\text { Fish } \\
\text { Remains }\end{array}$ & $\begin{array}{l}\text { Parasitic } \\
\text { Animals }\end{array}$ & Crustacea & $\begin{array}{l}\text { Other } \\
\text { Animals }\end{array}$ \\
\hline & $\begin{array}{l}\text { Mar. } \\
19{ }^{\circ} \\
65\end{array}$ & $\begin{array}{l}\text { Green } \\
\text { Island }\end{array}$ & & & & - & & & Forams $(24)$ \\
\hline
\end{tabular}

Parasitic Animals:

lots: 3 insect remains

Notes on Sqquid:

Notes on Fish:

General Notes: 1. Numerous rocks \& stones and small bits of insect particles in debris. 2. Forams of various sizes and types. 
SOSC REF. NO. 204

Sta. No. $20456 \quad$ Species Gyzis alba

POBSP. NO. 8

\begin{tabular}{|c|c|c|c|c|c|c|c|c|c|}
\hline $\begin{array}{l}\text { Lar S } \\
\text { Long. }\end{array}$ & Date & $\begin{array}{l}\text { Time } \\
\text { Caughe }\end{array}$ & Squita & $\begin{array}{l}\text { Squid } \\
\text { Beaks }\end{array}$ & Fish & $\begin{array}{c}\text { Fish } \\
\text { Remains }\end{array}$ & $\begin{array}{l}\text { Parasitic } \\
\text { Animals }\end{array}$ & Crustacea & $\begin{array}{l}\text { Other } \\
\text { Animals }\end{array}$ \\
\hline & $\begin{array}{l}\mathrm{Mar} . \\
24.2 \\
65\end{array}$ & $\begin{array}{l}\text { Green } \\
\text { Is Iand }\end{array}$ & & 3 & & - & & & \\
\hline
\end{tabular}

Parasitic Animals:

lots: 2 squid beaks

Notes on Squid:

debris

Notes on Fish:

General Notes:

Mostly milk weed is in the debris.

PACIFIC OCEANOGRAPHIC BIOLOGICAL SURVEY PROGRAM

SWITHSONIAN OCEANOGRAPHIC SORTING CENTER—SMITHSONIAN INSTITUTION

51. U5พ

S. Willis $3 / 2 / 66$

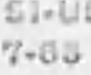

\begin{tabular}{|c|c|c|c|c|c|c|c|c|c|}
\hline Sta. No. & 204 & & Speci & & spo & & & $\begin{array}{l}\text { NO. } \\
\text { NO. }\end{array}$ & \\
\hline \multirow[t]{2}{*}{$\begin{array}{l}\text { Lat \&t } \\
\text { Long. }\end{array}$} & Date & $\begin{array}{l}\text { Time } \\
\text { Caught }\end{array}$ & Squid & $\begin{array}{l}\text { Squid } \\
\text { Beaks }\end{array}$ & Fish & $\begin{array}{c}\text { Fish } \\
\text { Remains }\end{array}$ & $\begin{array}{l}\text { Parasitic } \\
\text { Animals }\end{array}$ & Crustacea & $\begin{array}{l}\text { Other } \\
\text { Animals }\end{array}$ \\
\hline & $\begin{array}{l}\text { Apr. } \\
13 \\
65\end{array}$ & $\begin{array}{l}\text { Green } \\
\text { Island }\end{array}$ & & & & + & & & Foram (I) \\
\hline
\end{tabular}

Parasitic Animals:

Notes on Squid:

Iots: 2 foram fish remains

Notes on Fikh:

The fish remains are bones.

General Notes: 
SOSC REF. NO. 204

Sta., No. 25021

Species Anous stolidus

POBSP. NO.

\begin{tabular}{|c|c|c|c|c|c|c|c|c|c|}
\hline $\begin{array}{l}\text { Lat is } \\
\text { Long. }\end{array}$ & Date & $\begin{array}{l}\text { Time } \\
\text { Caught }\end{array}$ & Squid & $\begin{array}{l}\text { Squid } \\
\text { Beaks }\end{array}$ & Fish & $\begin{array}{l}\text { Fish } \\
\text { Remains }\end{array}$ & $\begin{array}{l}\text { Parasitic } \\
\text { Animals }\end{array}$ & Crustacea & $\begin{array}{l}\text { Other } \\
\text { Animals }\end{array}$ \\
\hline & $\begin{array}{l}\text { Aug. } \\
20 \text {, } \\
64\end{array}$ & $\begin{array}{l}\text { Sand } \\
\text { Is Iand }\end{array}$ & Remain. & & & + & & & \\
\hline
\end{tabular}

Parasitic Animals:

lots: 2 squid remains

Notes on Squid:

Notes on Fish:

General Notes:

PACIFIC OCEANOGRAPHIC BIOLOGICAL SURVEY PROGRAM

SMITHISONIAN OCEANOGRAPHIC SORTING CENTER_-SMITHSONIAN INSTITUTION

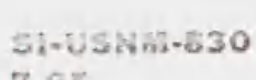

S. Willis $2 / 7 / 66$

7.05

\begin{tabular}{|c|c|c|c|c|c|c|c|c|c|}
\hline Sta. No. & 25025 & & Species & Stez & fus & & & $\begin{array}{l}\text { NO. } \\
\text { NO. }\end{array}$ & \\
\hline \multirow[t]{2}{*}{$\begin{array}{l}\text { Lat \& } \\
\text { Long. }\end{array}$} & Date & $\begin{array}{l}\text { Time } \\
\text { Caught }\end{array}$ & Squid & $\begin{array}{l}\text { Squid } \\
\text { Beaks } \\
\end{array}$ & Fish & $\begin{array}{c}\text { Fish } \\
\text { Remains }\end{array}$ & $\begin{array}{l}\text { Parasitic } \\
\text { Animals }\end{array}$ & Crustacea & $\begin{array}{l}\text { Other } \\
\text { Animals }\end{array}$ \\
\hline & $\begin{array}{l}\text { Aus. } \\
\frac{71}{64} \text {, }\end{array}$ & $\begin{array}{l}\text { Sand } \\
\text { Island }\end{array}$ & & & & - & & & \\
\hline
\end{tabular}

Parasitic Animals:

lots: 0

Notes on Squid:

Notes on Wish:

General Notes:

The stomach was empty. 


\begin{tabular}{|c|c|c|c|c|c|c|c|c|c|}
\hline $\begin{array}{l}\text { Lat \& } \\
\text { Long. }\end{array}$ & Date & $\begin{array}{l}\text { Time } \\
\text { Caught }\end{array}$ & Sc 4 & $\begin{array}{l}\text { Squid } \\
\text { Beaks }\end{array}$ & Fish & $\begin{array}{l}\text { Fish } \\
\text { Remains }\end{array}$ & $\begin{array}{l}\text { Parasitic } \\
\text { Animals }\end{array}$ & Crustacea & $\begin{array}{c}\text { Other } \\
\text { Animals }\end{array}$ \\
\hline & $\begin{array}{l}\text { Aug. } \\
18 \\
64\end{array}$ & $\begin{array}{l}\text { Sand } \\
\text { Is Iand }\end{array}$ & & & & + & & & \\
\hline
\end{tabular}

Parasitic Arimals:

lots: 2 Pish remains

Notes on Squid:

Notes on Wish:

The fish renains are bones.

Gencral Notes:

The digested remsins are beyond identification.

PACIFIC OCEANOGRAPHIC BIOLOGICAL SURVEY PROGRAM

SMITHSONIAN OCEANOGRAPHIC SORTING CENTER—SMITHSONIAN INSTITUTION

Ti*25sinaso

$\rightarrow 05$

S. Willis $1 / 26 / 66$

Sta. No. 25036

Species

Sterna fuscata

SOSC REF. NO. 204

POBSP. NO. 8

\begin{tabular}{|c|c|c|c|c|c|c|c|c|c|}
\hline $\begin{array}{l}\text { Lat \&s } \\
\text { Long. }\end{array}$ & Date & $\begin{array}{l}\text { Time } \\
\text { Caught }\end{array}$ & Squid & $\begin{array}{l}\text { Squid } \\
\text { Beaks }\end{array}$ & Fish & $\begin{array}{c}\text { Fish } \\
\text { Remains }\end{array}$ & $\begin{array}{l}\text { Parasitic } \\
\text { Animals }\end{array}$ & Crustacea & $\begin{array}{l}\text { Other } \\
\text { Animals }\end{array}$ \\
\hline & $\begin{array}{l}\text { Aug. } \\
15 \\
64\end{array}$ & $\begin{array}{l}\text { Sand } \\
\text { Island }\end{array}$ & & & & - & & & \\
\hline
\end{tabular}

Notes on Squid:

Iots: 1 digested remains

Notes on Fish:

General Notes:

The remains are beyond identification. 


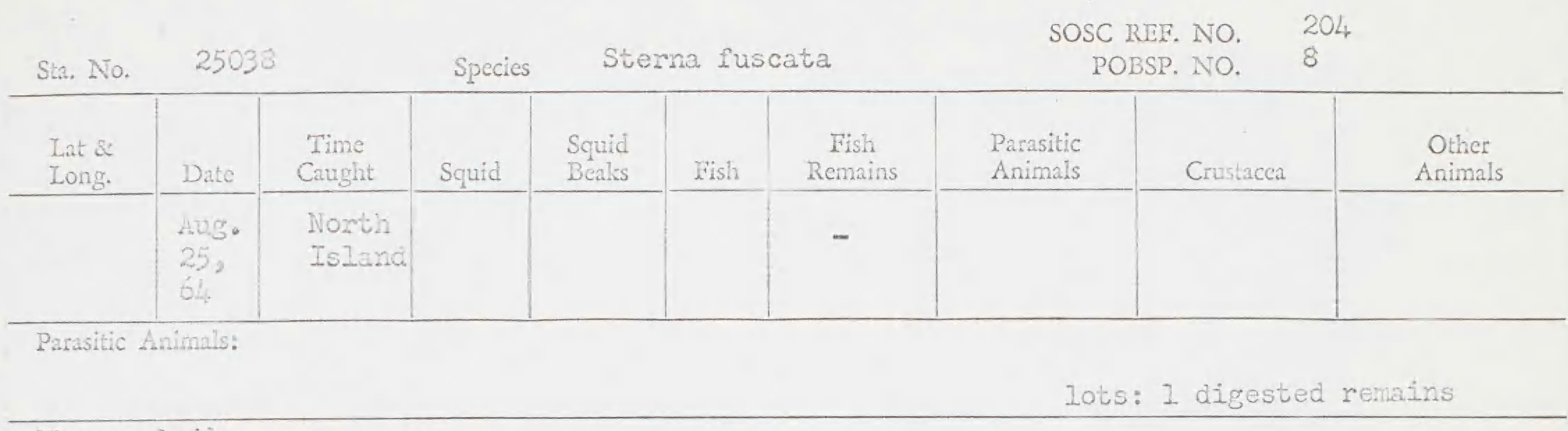

Notes on Squid:

Notes on Fish:

Genera Notes:

The remains are beyond identification.

PACIFIC OCEANOGRAPHIC BIOLOGICAL SURVEY PROGRAM

SMITHSONIAN OCEANOGRAPHIC SORTING CENTER -SMITHSONIAN INSTITUTION

Si-Usititu-330

S. Wi\}lis $1 / 26 / 66$

$7 \cdot 5.4$

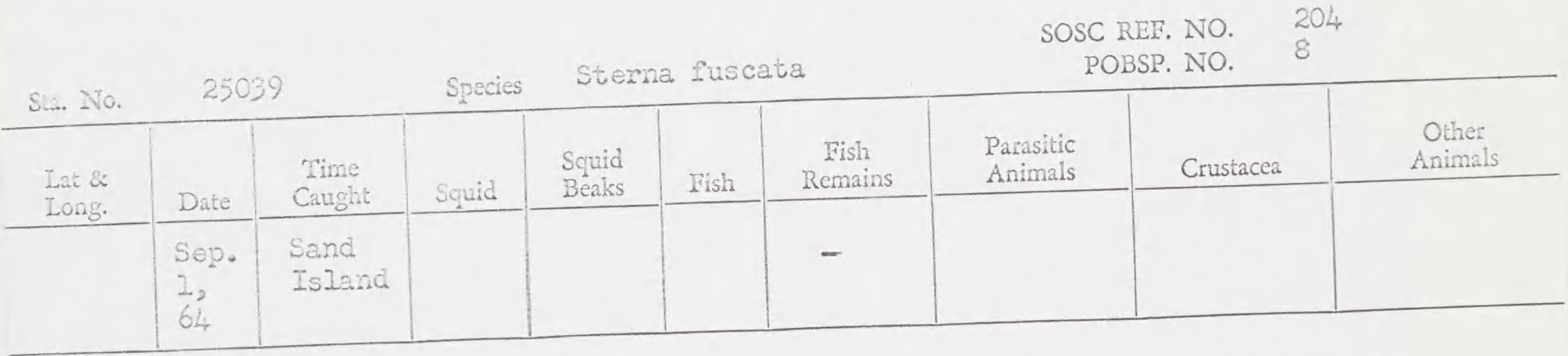

Parasitic Animals:

lots: 1 digested remains

Notes on Squid:

Notes on Fish:

General Notes:

The di jested remains are beyond identification.

PACIFIC OCEANOGRAPHIC BIOLOGICAL SURVEY PROGRAM

SMITHSONIAN OCEANOGRAPHIC SORTING CENTER—SMITHSONIAN INSTITUTION

S. Willis $2 / 7 / 66$

S1-45พแ-630

$7+68$ 
SOSC REF. NO. 204

Sta. No. $\quad 30036$

Species Procelsterna cemilea

POBSP. NO. 8

\begin{tabular}{|c|c|c|c|c|c|c|c|c|c|}
\hline $\begin{array}{l}\text { Lat \&s } \\
\text { Long. }\end{array}$ & Date & $\begin{array}{l}\text { Time } \\
\text { Caught }\end{array}$ & Squid & $\begin{array}{l}\text { Squid } \\
\text { Beaks } \\
\end{array}$ & Fish & $\begin{array}{c}\text { Fish } \\
\text { Remains }\end{array}$ & $\begin{array}{l}\text { Parasitic } \\
\text { Animals }\end{array}$ & Crustacea & $\begin{array}{l}\text { Other } \\
\text { Animals }\end{array}$ \\
\hline & $\begin{array}{l}\text { Oet. } \\
77^{2} \\
64\end{array}$ & $\begin{array}{l}\text { Marsha11 } \\
\text { Island }\end{array}$ & & & & - & & & \\
\hline
\end{tabular}

Parasitic Animals:

lots: 1 debris

Notes on Squid:

Notes on Fish:

General Notes:

Tiny bits of a wood-like material are in the debris.

PACIFIC OCEANOGRAPHIC BIOLOGICAL SURVEY PROGRAM

SMITHSONIAN OCEANOGRAPHIC SORTING CENTER—SMITHSONIAN INSTITUTION

Si-USNW-630

7.65

S. Willis $2 / 2 / 66$

SOSC REF. NO. 204

\begin{tabular}{|c|c|c|c|c|c|c|c|c|c|}
\hline $\begin{array}{l}\text { Lat \& } \\
\text { Long. }\end{array}$ & Date & $\begin{array}{l}\text { Time } \\
\text { Caught }\end{array}$ & Squid & $\begin{array}{l}\text { Squid } \\
\text { Beaks } \\
\end{array}$ & Fish & $\begin{array}{c}\text { Fish } \\
\text { Remains }\end{array}$ & $\begin{array}{l}\text { Parasitic } \\
\text { Animals } \\
\end{array}$ & Crustacea & $\begin{array}{l}\text { Other } \\
\text { Animals }\end{array}$ \\
\hline & $\begin{array}{l}\text { Oet. } \\
64\end{array}$ & $\begin{array}{l}\text { MarsheI } \\
\text { Island }\end{array}$ & Remais & & 23 & + & & Crabs (2) & $\begin{array}{l}\text { Stomatopod } \\
(1) \\
\text { plus } 3\end{array}$ \\
\hline
\end{tabular}

Parasitic Animals:

Lots: 6 squid remains

Now ow Syad: Iots (A) are the stomach contents of 30079. Lots (B) are separate contents from a plastic bag with the same field no. The Notes on Fish:

a vial.

Geneml Notes:

Part of the stomatopod's abdomen and appendages of lots (A) are detached.
Iish remains stomatopod

Pish

crabs

stomatopods
(A)

(B)

\section{PACIFIC OCEANOGRAPHIC BIOLOGICAL SURVEY PROGRAM}

SMITHSONIAN OCEANOGRAPHIC SORTING CENIER—SMITHSONIAN INSTITUTION 
SOSC REF. NO. 204 POBSP. NO.

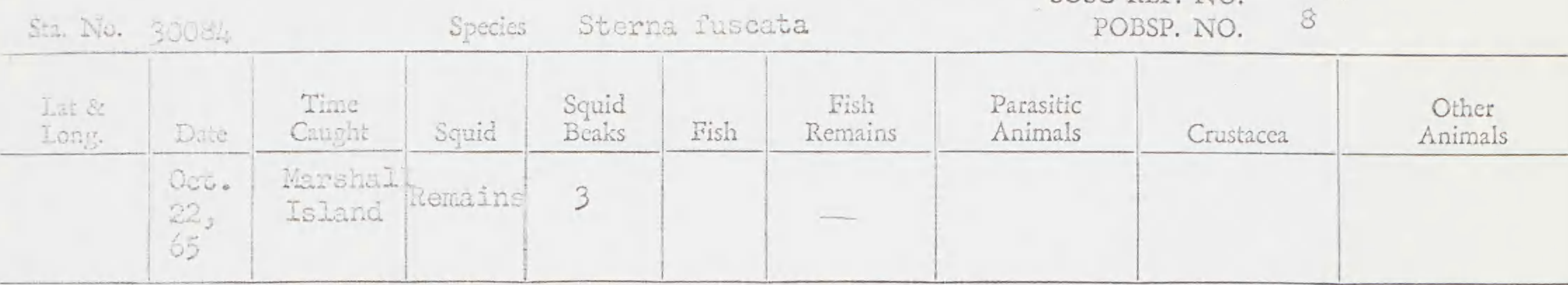

Purnsitic Aninats:

Iots: 3 squid beaks

Notes on Squid:

squid remains

digested remains

Notes on Fish:

Gencral Notes:

PACIEIC OCEANOGRAPHIC BIOLOGICAL SURVEY PROGRAM

SMITHSONIAN OCEANOGRAPHIC SORTING CENTER — SMITHSONIAN INSTITUTION

S. Wiltis $1 / 26 / 66$

S1-USNM-330

7.65

SOSC REF. NO. 204

Sta. No.

Species

POBSP. NO. 8

\begin{tabular}{|c|c|c|c|c|c|c|c|c|c|}
\hline $\begin{array}{l}\text { Lat \& } \\
\text { Long. }\end{array}$ & Date & $\begin{array}{l}\text { Time } \\
\text { Caught }\end{array}$ & Squid & $\begin{array}{l}\text { Squid } \\
\text { Beaks }\end{array}$ & Fish & $\begin{array}{c}\text { Fish } \\
\text { Remains }\end{array}$ & $\begin{array}{l}\text { Parasitic } \\
\text { Animals }\end{array}$ & Crustacea & $\begin{array}{l}\text { Other } \\
\text { Animals }\end{array}$ \\
\hline & $\begin{array}{l}\text { Oct. } \\
24,3 \\
64 .\end{array}$ & $\begin{array}{l}\text { Marsha11 } \\
\text { Island }\end{array}$ & & & & - & & Remains & $\begin{array}{l}\text { Gestropods }(79) \\
\text { Forans (31) }\end{array}$ \\
\hline
\end{tabular}

Parasitic Animals:

Lots: 4 gastropods

Notes on Squid:

forams

crustacea remains

debris

Notes on Fish:

General Notes: 1. The crublacea remains are bits of an isopod.

2. The gastropods are of various sizes. 
SOSC REF. NO. 204

Sta. No. 30103

Species

Awenaria interpres POBSP. NO.

8

\begin{tabular}{|c|c|c|c|c|c|c|c|c|c|}
\hline $\begin{array}{l}\text { Tat \& } \\
\text { Iong. }\end{array}$ & Date & $\begin{array}{l}\text { Time } \\
\text { Crught }\end{array}$ & Scuic & $\begin{array}{l}\text { Squid } \\
\text { Beaks }\end{array}$ & Fish & $\begin{array}{c}\text { Fish } \\
\text { Remains } \\
\end{array}$ & $\begin{array}{l}\text { Parasitic } \\
\text { Animals }\end{array}$ & Crustaccá & $\begin{array}{l}\text { Other } \\
\text { Animals }\end{array}$ \\
\hline & $\begin{array}{l}004 \\
245 \\
64\end{array}$ & $\begin{array}{l}\text { Marshe } 11 \\
\text { Island }\end{array}$ & & & & - & & $\begin{array}{l}\text { Isopods (2) } \\
\text { Remains }\end{array}$ & $\begin{array}{l}\text { Castropods } \\
\qquad(59) \\
\text { Forams (7) } \\
\text { Insects (3) }\end{array}$ \\
\hline
\end{tabular}

n.witichamals: The abdomens of two insects are detached.

lots: 7 isopods

Nom on squit: The cmutaced renains are mostly bits of isopods. crustacea remains gastropods Poraminifers

Notes on Fish: insects

jnsect renains dehris

Genenl Notes: The gastropods are of various sizes.

PACIFIC OCEANOGRAPHIC BIOLOGICAL SURVEY PROGRAM

SMITHSONIAN OCEANOGRAPHIC SORTING CENTER_SMITHSONIAN INSTITUTION $2 / 2 / 66$

Si.USNai-230

$7 \cdot 05$

SOSC REF. NO. 204

Sta. No. 30111

Sterna sumatrana POBSP. NO.

\begin{tabular}{|c|c|c|}
\hline $\begin{array}{l}\text { Lat \& } \\
\text { Long. }\end{array}$ & Date & $\begin{array}{l}\text { Time } \\
\text { Caught }\end{array}$ \\
\hline & $\begin{array}{l}\text { Oct. } \\
25, \\
64,\end{array}$ & $\begin{array}{l}\text { Harshall } \\
\text { Is land }\end{array}$ \\
\hline
\end{tabular}

Species

\begin{tabular}{|c|c|} 
Fish & $\begin{array}{c}\text { Fish } \\
\text { Remains }\end{array}$ \\
+
\end{tabular}

\begin{tabular}{|l|c|c}
$\begin{array}{c}\text { Parasitic } \\
\text { Animals }\end{array}$ & Crustacea & $\begin{array}{c}\text { Othes } \\
\text { Animals }\end{array}$ \\
\hline & &
\end{tabular}

Parasitic Animals:

lots: 1. Iish remains

Notes on Squid:

Notes on Fish:

Remains are mostly bones.

General Notes: 


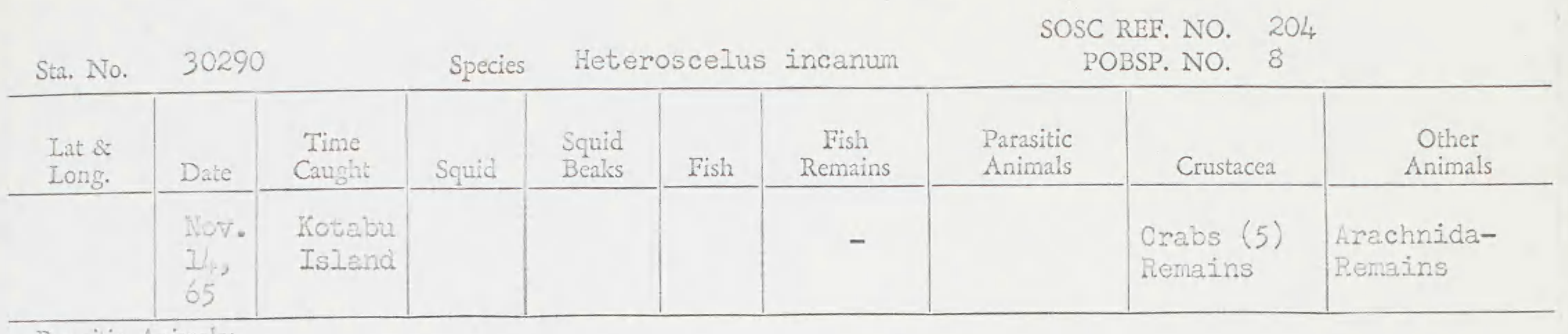

Parasitic Animals:

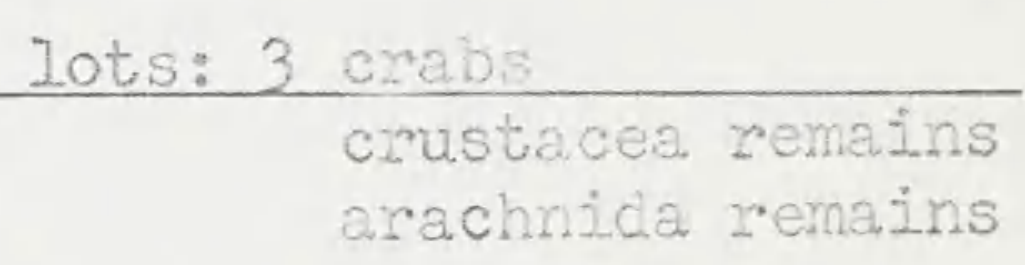

The arachnid remains consist of an abdomen and appendages of a spider.

General Notes:

1. One of the crabs is a larval stage.

2. The crustacea remains consist oi claws, legs, and bits of shell.

PACIFIC OCEANOGRAPHIC BIOLOGICAL SURVEY PROGRAM

SMITHSONIAN OCEANOGRAPHIC SORTING CENTER_ -SMITHSONIAN INSTITUTION

S. Willis $2 / 6 / 66$

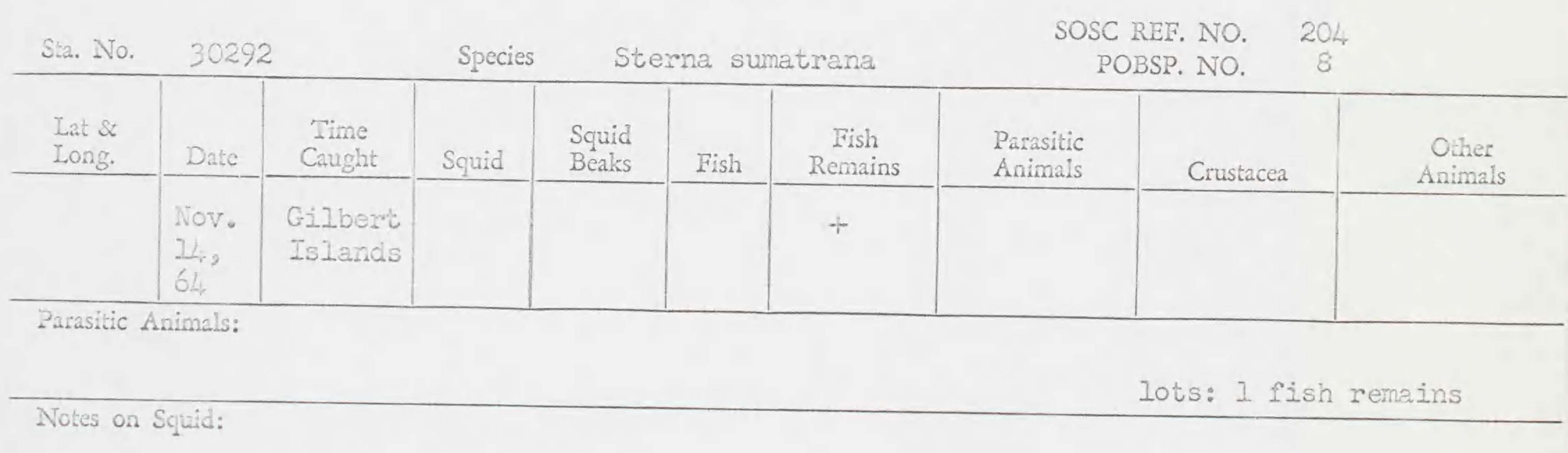

Notes on Fish:

fiematins are bones.

General Notes: 
SOSC REF. NO. 204

\begin{tabular}{|c|c|c|c|c|c|c|c|c|c|}
\hline Sta. No & & & Speci & & 1a: & trana & & NO. & \\
\hline $\begin{array}{l}\text { Lat \& } \\
\text { Long. }\end{array}$ & Date & $\begin{array}{l}\text { Time } \\
\text { Caught }\end{array}$ & Squid & $\begin{array}{l}\text { Squid } \\
\text { Bcaks }\end{array}$ & Fish & $\begin{array}{c}\text { Fish } \\
\text { Remains }\end{array}$ & $\begin{array}{l}\text { Parasitic } \\
\text { Animals }\end{array}$ & Crustacea & $\begin{array}{l}\text { Other } \\
\text { Animals }\end{array}$ \\
\hline & $\begin{array}{l}\text { Nov. } \\
\frac{1}{6 t} 0\end{array}$ & $\begin{array}{l}\text { Cillbert } \\
\text { Islands }\end{array}$ & & & & + & & & $\begin{array}{c}\text { astropod (1) } \\
\text { foraminifera } \\
\text { (131) }\end{array}$ \\
\hline
\end{tabular}

Parasitic Animals:

Lots: 3 fish renains

Notes on Squid:

foraminifera

Notes on Fibh: Small vits of rocks \& stones are mixed with the fish remains.

General Notes: The foraminifera are of vaxious sizes and only of two or three different types.

PACIFIC OCEANOGRAPHIC BIOLOGICAL SURVEY PROGRAM

SMITHISONIAN OCEANOGRAPHIC SORTING CENTER—SMITHSONIAN INSTITUTION

S. Wi1zis $2 / 2 / 66$

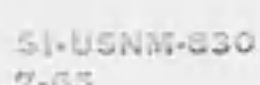

7.05

SOSC REF. NO. 204

POBSP. NO. 8

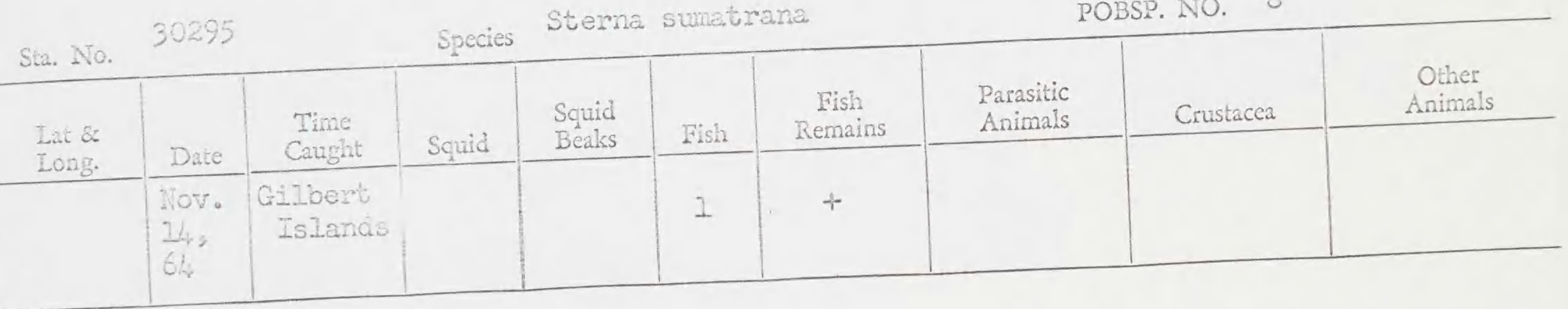

Parasitic Animals:

Iotsi 2 fish remains

fish

Notes on Squid:

Notes on Fish:

The .t. the fish is digested.

General Notes:

PACIFIC OCEANOGRAPHIC BIOLOGICAL SURVEY PROGRAM SMITHSONIAN OCEANOGRAPHIC SORTING CENTER-SMITSONIAN INSTITUIION 
Sta. No. 30296

\begin{tabular}{|c|c|c|}
\hline $\begin{array}{l}\text { Lat \& } \\
\text { Long. }\end{array}$ & Date & $\begin{array}{l}\text { Time } \\
\text { Caught }\end{array}$ \\
\hline & $\begin{array}{l}\text { Nov, } \\
16, \\
64\end{array}$ & $\begin{array}{l}\text { Gilibert } \\
\text { Islands }\end{array}$ \\
\hline
\end{tabular}

Parasitic Animals:
SOSC REF. NO. POBSP. NO.
204

8
Species sterna sumatrana

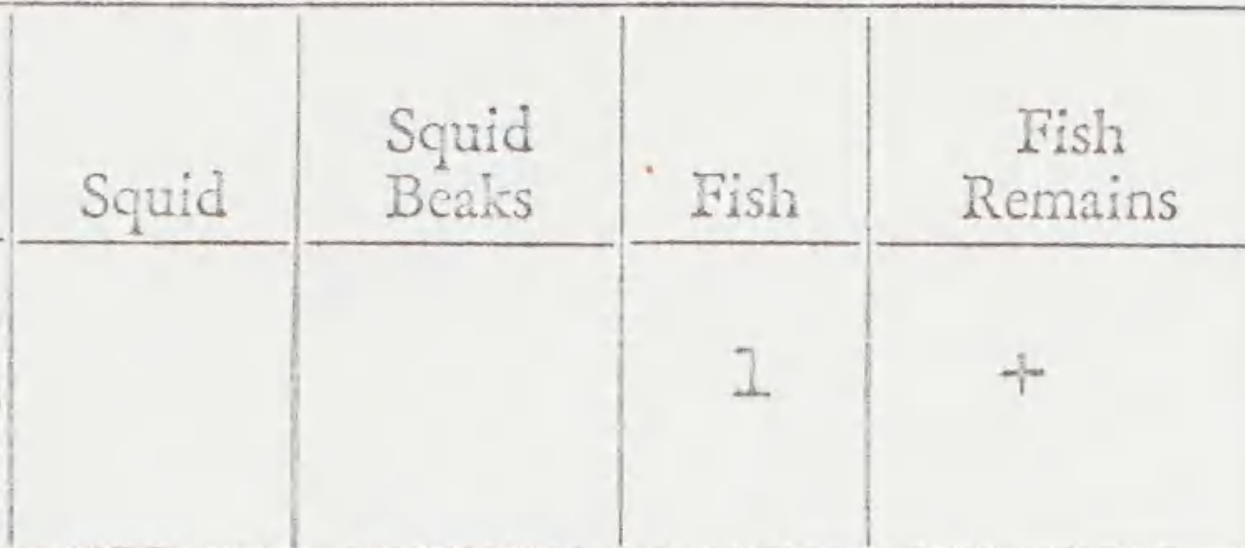

$\left|\begin{array}{c}\text { Parasitic } \\ \text { Animals }\end{array}\right| \begin{gathered}\text { Other } \\ \text { Animals }\end{gathered}$

lots: 2 fish

Notes on Scuid:

Notes on Fish: 1. The Pish's head is digested.

2. The fish remains are bones.

General Notes:

PACIFIC OCEANOGRAPHIC BIOLOGICAL SURVEY PROGRAM

SMITHSONIAN OCEANOGRAPHIC SORTING CENTER-SMITHSONIAN INSTITUTION

Si-USNM-830

S. Willis $2 / 7 / 66$

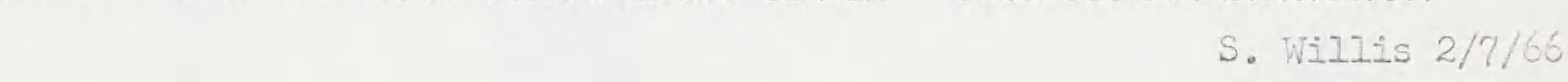

Sca. No. 30297

Species Sterna sumatrana

SOSC REF. NO,

204 POBSP. NO.

\begin{tabular}{|c|c|c|c|c|c|c|c|c|c|}
\hline $\begin{array}{l}\text { Lat \&c } \\
\text { Long. }\end{array}$ & Date & $\begin{array}{l}\text { Time } \\
\text { Caught }\end{array}$ & Squid & $\begin{array}{l}\text { Squid } \\
\text { Beaks }\end{array}$ & Fish & $\begin{array}{c}\text { Fish } \\
\text { Remains }\end{array}$ & $\begin{array}{l}\text { Parasitic } \\
\text { Animals }\end{array}$ & Crustacea & $\begin{array}{l}\text { Other } \\
\text { Animals }\end{array}$ \\
\hline & $\begin{array}{l}\text { Not. } \\
1142 \\
64\end{array}$ & $\begin{array}{l}\text { Gilbert } \\
\text { Islands }\end{array}$ & & & & + & & & \\
\hline
\end{tabular}

Parasitic Animals:

Lots: 1 fish remains

Notes on Squid:

Wotes on Hin...

Gencial Notes: 


\begin{tabular}{|c|c|c|c|c|c|c|c|c|c|}
\hline Sta. No. & 30313 & & Specie & $\mathrm{Heb}$ & celu & incanum & & $\begin{array}{l}\text { NO. } \\
\text { NO. }\end{array}$ & \\
\hline \multirow[t]{2}{*}{$\begin{array}{l}\text { Lat } 8 x \\
\text { Long. }\end{array}$} & Date & $\begin{array}{l}\text { Time } \\
\text { Caught } \\
\end{array}$ & Squid & $\begin{array}{l}\text { Squid } \\
\text { Beaks }\end{array}$ & Fish & $\begin{array}{c}\text { Fish } \\
\text { Remains }\end{array}$ & $\begin{array}{l}\text { Parasitic } \\
\text { Animals }\end{array}$ & Crustacea & $\begin{array}{l}\text { Other } \\
\text { Animals }\end{array}$ \\
\hline & $\begin{array}{l}\text { Nov. } \\
\frac{148}{64}\end{array}$ & $\begin{array}{l}\text { Gilbent } \\
\text { Islands }\end{array}$ & & & & - & & & \\
\hline
\end{tabular}

Parasitic Animals:

Jots: I digested remains

Notes on Squid:

Notes on Fish:

The remains are beyond identification except for one crustacean head.

General Notes:

PACIFIC OCEANOGRAPHIC BIOLOGICAL SURVEY PROGRAM

SMITHSONIAN OCEANOGRAPHIC SORTING CENTER-SMITHSONIAN INSTITUTION 


\begin{tabular}{|c|c|c|c|c|c|c|c|c|c|}
\hline Sta. No. & $203 \%$ & & Speci & Fre & $a m$ & & SOS & $\begin{array}{l}\text { NO. } \\
\text { NO. }\end{array}$ & \\
\hline \multirow[t]{2}{*}{$\begin{array}{l}\text { Lat \& } \\
\text { Long. }\end{array}$} & Date & $\begin{array}{l}\text { Time } \\
\text { Caught }\end{array}$ & Squid & $\begin{array}{l}\text { Squid } \\
\text { Beaks }\end{array}$ & Fish & $\begin{array}{c}\text { Eish } \\
\text { Remains }\end{array}$ & $\begin{array}{l}\text { Parasitic } \\
\text { Animals }\end{array}$ & Crustacea & $\begin{array}{l}\text { Other } \\
\text { Animals }\end{array}$ \\
\hline & $\begin{array}{l}\text { Mova } \\
3 \% \\
64\end{array}$ & $\begin{array}{l}\text { Green } \\
\text { Island }\end{array}$ & & 56 & & - & $\begin{array}{l}\text { Nematodes } \\
\text { (2) }\end{array}$ & & \\
\hline
\end{tabular}

Parasitic Animals:

Iots: 4

Notes on Squid: nematodes debris digested remains

Notes on Fish:

General Notes: I. The remains are beyond identification.

2. The debris is some type of top ie., toothpaste or hair creame.

PACIFIC OCEANOGRAPHIC BIOLOGICAL SURVEY PROGRAM SMITHSONIAN OCEANOGRAPHIC SORTING CENTER_-SMITHSONIAN INSTITUTION 


\begin{tabular}{|c|c|c|c|c|c|c|c|c|}
\hline $\begin{array}{l}\text { *Fish } \\
\text { by }+ \text { ? }\end{array}$ & $\begin{array}{l}\text { remains i } \\
\text { o* - sign }\end{array}$ & ndicate & & & & SOS & $\begin{array}{l}\text { REF. NO. } \\
\text { POBSP NO. }\end{array}$ & $\begin{array}{l}\text { p. I of } \\
204 \\
8\end{array}$ \\
\hline Sta 110. & Species & Sguid & $\begin{array}{l}\text { Squid } \\
\text { bealks }\end{array}$ & Fish & $\begin{array}{l}\text { Fish } \\
\text { Rern.* }\end{array}$ & $\begin{array}{l}\text { Faresitic } \\
\text { Animals }\end{array}$ & Crustacea & $\begin{array}{l}\text { Other } \\
\text { Animals }\end{array}$ \\
\hline 0520 & $\begin{array}{l}\text { cuero- } \\
\text { coma } \\
\text { tterna }\end{array}$ & & 28 & & + & & & \\
\hline 2051 & $\begin{array}{l}\text { bulweria } \\
\text { bulweriti }\end{array}$ & & & & - & & & \\
\hline 2052 & $\begin{array}{l}\text { Stema } \\
\text { Puscata }\end{array}$ & & 3 & & - & & & \\
\hline 2053 & $\begin{array}{l}\text { terro- } \\
\text { mintis } \\
\text { cauda }\end{array}$ & 1. & & & + & & & \\
\hline 2054 & $\begin{array}{l}\text { Sula } \\
\text { dactyla- } \\
\text { tra }\end{array}$ & & 19 & & - & & & \\
\hline 3056 & $\begin{array}{l}\text { Ptero- } \\
\text { croma } \\
\text { xterna }\end{array}$ & & 128 & & - & Nematocia (I) & & \\
\hline 1057 & $\begin{array}{l}\text { ula } \\
\text { actyla- } \\
\text { ura }\end{array}$ & & & & + & & & \\
\hline 2058 & $\begin{array}{l}\text { difro- } \\
\text { externa }\end{array}$ & & 267 & & - & & & \\
\hline 1059 & $\begin{array}{l}\text { wero- } \\
\text { externa }\end{array}$ & & 23 & & + & $\begin{array}{c}\text { Nematode } \\
\text { (1) }\end{array}$ & & \\
\hline 1060 & $\begin{array}{l}\text { Sula } \\
\text { dactyla- } \\
\text { tra }\end{array}$ & & 1 & & + & & & \\
\hline 1062 & $\begin{array}{l}\text { arora- } \\
\text { externa } \\
\end{array}$ & & 304 & & - & & & \\
\hline 1062 & $\begin{array}{l}\text { putimus } \\
\text { pacifi- } \\
\text { cus }\end{array}$ & & & & - & & & \\
\hline 1063 & $\mid \begin{array}{l}\text { puftinus } \\
\text { pacificus }\end{array}$ & & & & - & $\begin{array}{c}\text { Nematodes } \\
(2)\end{array}$ & & \\
\hline 1064 & $\begin{array}{l}\text { Uceano- } \\
\text { roma } \\
\text { Ieucorho }\end{array}$ & & & & - & & & \\
\hline 1065 & $\begin{array}{l}\text { trero- } \\
\text { droma } \\
\text { externa }\end{array}$ & & & & + & & & \\
\hline 1066 & $\begin{array}{l}\text { Ptero- } \\
\text { irona } \\
\text { externa }\end{array}$ & me- & & 1 & + & $\begin{array}{c}\text { Nematode } \\
\text { (I) }\end{array}$ & & 20 \\
\hline 7.068 & $\begin{array}{l}\text { aect } \\
\text { tret }\end{array}$ & 3 & 1 & & - & & & . \\
\hline 1069 & $\begin{array}{l}\text { Sterna } \\
\text { fuscata }\end{array}$ & 4 & 4 & 1 & + & & & \\
\hline
\end{tabular}


* Fish remains indicated by $+o r$ - sign
SOSC REF. NO. 204 POBSP INO. 8

\begin{tabular}{|c|c|c|c|c|c|c|c|c|}
\hline Sta No. & Species & Squia & $\begin{array}{l}\text { Squia } \\
\text { beaks }\end{array}$ & Fish & $\begin{array}{l}\text { Fish } \\
\text { Rem.* }\end{array}$ & $\begin{array}{l}\text { Paresitio } \\
\text { pimals }\end{array}$ & Crustacea & $\begin{array}{l}\text { Other } \\
\text { Animals }\end{array}$ \\
\hline 1070 & $\begin{array}{l}\text { Sterna } \\
\text { fuscata }\end{array}$ & Remains & & & - & & & \\
\hline 1071 & $\begin{array}{l}\text { Puffinus } \\
\text { pacificus }\end{array}$ & 8 & & & - & & & \\
\hline 1072 & $\begin{array}{l}\text { Ptero- } \\
\text { droma } \\
\text { externa }\end{array}$ & & 22 & & - & & & \\
\hline 1073 & $\begin{array}{l}\text { Sterna } \\
\text { fuscata }\end{array}$ & 4 & & & - & & & \\
\hline 1074 & $\begin{array}{l}\text { Stema } \\
\text { fuscata }\end{array}$ & 2 & & 2 & + & & & \\
\hline 1075 & $\begin{array}{l}\text { Sterna } \\
\text { fuscata }\end{array}$ & 1 & & & - & & & \\
\hline 1077 & $\begin{array}{l}\text { Sula } \\
\text { dactyl- } \\
\operatorname{tra}\end{array}$ & & 7 & & + & & & \\
\hline 1079 & $\begin{array}{l}\text { Sula } \\
\text { dactyla } \\
\text { tra }\end{array}$ & & 15 & & + & & & \\
\hline 1080 & $\begin{array}{l}\text { Ptero- } \\
\text { droma } \\
\text { externa }\end{array}$ & & & & - & & & \\
\hline 1081 & $\begin{array}{l}\text { Sula } \\
\text { dactyla- } \\
\text { tra }\end{array}$ & & & 1 & + & & & \\
\hline 1082 & $\begin{array}{l}\text { Stema } \\
\text { fuscata }\end{array}$ & & & & - & & & \\
\hline 1083 & $\begin{array}{l}\text { Sula } \\
\text { dactyla- } \\
\text { tra }\end{array}$ & $\begin{array}{c}3 \\
\text { Re- } \\
\text { mains }\end{array}$ & 34 & 1 & + & & $\begin{array}{l}\text { Isopods } \\
\text { (2) }\end{array}$ & \\
\hline 1085 & $\begin{array}{l}\text { Sula } \\
\text { dactyla- } \\
\text { tra }\end{array}$ & & 14 & & + & & $\begin{array}{r}\text { Isopod } \\
(1)\end{array}$ & \\
\hline 1086 & $\begin{array}{l}\text { Ptero- } \\
\text { droma } \\
\text { externa }\end{array}$ & mains & 296 & & + & & & \\
\hline 1087 & $\begin{array}{l}\text { Stercor- } \\
\text { grius } \\
\text { Iongicaud }\end{array}$ & a & & & - & & & $\begin{array}{l}\text { Eggs } \\
\text { (I) }\end{array}$ \\
\hline 1088 & $\begin{array}{l}\text { Sula } \\
\text { dacty.la- } \\
\text { bra } \\
\text { Stema }\end{array}$ & & 2 & & + & & & \\
\hline 1089 & $\left\{\begin{array}{l}\text { Sterna } \\
\text { fuscata }\end{array}\right.$ & & 2 & 1 & + & & ? & \\
\hline 1090 & $\begin{array}{l}\text { Sterna } \\
\text { fuscatá }\end{array}$ & & & 1 & + & & & \\
\hline
\end{tabular}


*2. Sh remeins indicated

by + or - sign p. 3 of 10

SOSC REF. NO. 204

POBSP NO. 8

\begin{tabular}{|c|c|c|c|c|c|c|c|c|}
\hline Sta No. & Species & Souid & $\begin{array}{l}\text { Squia } \\
\text { beaks }\end{array}$ & Fish & $\begin{array}{l}\text { Fish } \\
\text { Rem.* }\end{array}$ & $\begin{array}{l}\text { Parssitic } \\
\text { Animals }\end{array}$ & Crustacea & $\begin{array}{l}\text { Other } \\
\text { Animals }\end{array}$ \\
\hline 1112 & $\begin{array}{l}\text { Phaethor } \\
\text { Iepturus }\end{array}$ & & 14 & 2 & + & $\begin{array}{l}\text { Nematodes }(2) \\
\text { Isopod (I) }\end{array}$ & $\begin{array}{l}\text { Copepod }(1) \\
\text { (parasitic }\end{array}$ & \\
\hline 1113 & $\begin{array}{l}\text { Sterna } \\
\text { fuscata. }\end{array}$ & & & & + & & & \\
\hline 1115 & $\begin{array}{l}\text { Sterna } \\
\text { fuscata }\end{array}$ & & & & - & & & \\
\hline 1216 & $\begin{array}{l}\text { Phaethor } \\
\text { lepturus }\end{array}$ & $\begin{array}{l}\text { Re- } \\
\text { mains }\end{array}$ & 11 & 4 & + & & & \\
\hline 1143 & $\begin{array}{l}\text { Sterna } \\
\text { fuscata }\end{array}$ & & & & - & & & \\
\hline 12.44 & $\begin{array}{l}\text { Sterna } \\
\text { fuscata }\end{array}$ & & & & - & & & \\
\hline 1149 & $\begin{array}{l}\text { Sterna } \\
\text { fuscata }\end{array}$ & & & & - & & & \\
\hline 1261 & $\begin{array}{l}\text { Puffinus } \\
\text { pacifi- } \\
\text { cus }\end{array}$ & 2 & & & + & $\begin{array}{l}\text { Nematode } \\
\text { (I) }\end{array}$ & & \\
\hline 1162 & $\begin{array}{l}\text { Puffinus } \\
\text { pacifi- } \\
\end{array}$ & & 87 & & + & & & \\
\hline 1164 & $\begin{array}{l}\text { Sterna } \\
\text { fuscata }\end{array}$ & & 7 & & + & & & \\
\hline 1265 & $\begin{array}{l}\text { Sterna } \\
\text { fuscata }\end{array}$ & $I$ & 4 & 1 & + & & & \\
\hline 2167 & $\begin{array}{l}\text { Puffinus } \\
\text { pucifi- } \\
\text { cus }\end{array}$ & $\begin{array}{l}\text { Re- } \\
\text { mains }\end{array}$ & 144 & & + & & & \\
\hline 1269 & $\begin{array}{l}\text { Sterna } \\
\text { fuscata }\end{array}$ & 2 & & & + & & & \\
\hline 2172 & $\begin{array}{l}\text { Puffinus } \\
\text { pacifi- } \\
\text { cus }\end{array}$ & $\begin{array}{l}\mathrm{Re}- \\
\operatorname{main}\end{array}$ & 88 & & + & & & \\
\hline 1172 & $\begin{array}{l}\text { Puffinus } \\
\text { pacifi- } \\
\text { pus }\end{array}$ & & 3 & & - & & & \\
\hline 1173 & $\begin{array}{l}\text { Puffinus } \\
\text { pacifi- } \\
\text { suc }\end{array}$ & & & & + & $\begin{array}{c}\text { Nematode } \\
\text { (1) }\end{array}$ & & \\
\hline 1175 & $\begin{array}{l}\text { Gyy is } \\
\text { alba }\end{array}$ & & & & + & & . & \\
\hline & $\begin{array}{l}\text { Puffinus } \\
\text { pacificus }\end{array}$ & & & & - & & & \\
\hline
\end{tabular}


Hish remains indicated

by + or $-\operatorname{sign}$
SOSC REF. NO. 204

POBSP NO. 8

\begin{tabular}{|c|c|c|c|c|c|c|c|c|}
\hline Sta No. & Species & squid & $\begin{array}{l}\text { Squiá } \\
\text { bealis }\end{array}$ & Fish & $\begin{array}{l}\text { Fish } \\
\text { Rem.* }\end{array}$ & $\begin{array}{l}\text { Puresitic } \\
\text { Anjunals }\end{array}$ & Crustacea & $\begin{array}{l}\text { Other } \\
\text { Animals }\end{array}$ \\
\hline 1091 & $\begin{array}{l}\text { dactyla- } \\
\text { taca }\end{array}$ & & 8 & & + & & & \\
\hline 1092 & $\begin{array}{l}\text { terna } \\
\text { fuscata }\end{array}$ & & & & + & & & \\
\hline 1093 & $\begin{array}{l}\text { Pufinus } \\
\text { nativita }\end{array}$ & Rè- & 99 & & + & & & \\
\hline 1094 & $\begin{array}{l}\text { Sula } \\
\text { dactyIa } \\
\text { tra }\end{array}$ & Re- & 10 & 2 & + & & 1 & \\
\hline 2095 & $\begin{array}{l}\text { Sula } \\
\text { dactyla } \\
\text { tra }\end{array}$ & Re- & 23 & 2 & + & & & \\
\hline 1096 & $\begin{array}{l}\text { Sula } \\
\text { dactyla- } \\
\text { tra }\end{array}$ & & 3 & 2 & \pm & & 1 & \\
\hline 1098 & $\begin{array}{l}\text { Sula } \\
\text { dactyla- } \\
\text { tra }\end{array}$ & & 2 & 2 & + & & & \\
\hline 1099 & $\begin{array}{l}\text { Sula } \\
\text { dactyla } \\
\text { tra }\end{array}$ & 2 & 28 & 1 & + & & & \\
\hline 1100 & $\begin{array}{l}\text { Sula } \\
\text { dactyla } \\
\text { tra }\end{array}$ & mains & 19 & & + & $\begin{array}{c}\text { Tapeworms } \\
\text { (5) }\end{array}$ & & \\
\hline 2101 & $\mid \begin{array}{l}\text { Sterna } \\
\text { fuscata }\end{array}$ & & & 2 & + & & & \\
\hline 1102 & $\begin{array}{l}\text { Puffinus } \\
\text { pacifi- } \\
\text { cus }\end{array}$ & $\begin{array}{c}7 \\
\text { Re- } \\
\text { mains }\end{array}$ & 96 & & - & & & \\
\hline 1103 & $\begin{array}{l}\text { Sula } \\
\text { dactyla- } \\
\text { tra }\end{array}$ & 4 & 22 & 2 & + & & & \\
\hline 1204 & $\begin{array}{l}\text { Sula } \\
\text { dactyla- } \\
\text { tera }\end{array}$ & $\begin{array}{l}\text { Re- } \\
\text { mains }\end{array}$ & 4 & & + & & $\begin{array}{l}\text { p. copepods } \\
\text { (2I) } \\
\text { copepod (I) }\end{array}$ & \\
\hline 1106 & $\begin{array}{l}\text { Sterna } \\
\text { fuscata }\end{array}$ & & & & - & & & \\
\hline 1207 & $\begin{array}{l}\text { dula } \\
\text { dactyla- }\end{array}$ & 1 & & & + & & & \\
\hline 1109 & $\begin{array}{l}\text { Sula } \\
\text {-iacty } 1 a- \\
\text { ra }\end{array}$ & $\begin{array}{c}10 \\
\text { Re- } \\
\text { mains }\end{array}$ & .11 & 3 & + & & & \\
\hline 1110 & $\begin{array}{l}\text { Puffinus } \\
\text { pacifi- }\end{array}$ & $i$ & & & - & & & \\
\hline 1111 & $\left\{\begin{array}{l}\text { SuIt } \\
\text { drctyla- } \\
\text { bee }\end{array}\right.$ & & & 3 & + & & & \\
\hline
\end{tabular}


* Fish remains indicated by + or - sign
SOSC REF. NO. 204

POBSP NO, 8

\begin{tabular}{|c|c|c|c|c|c|c|c|c|}
\hline Ste No. & Species & Squid & $\begin{array}{l}\text { Squid } \\
\text { beaks }\end{array}$ & Fish & $\begin{array}{l}\text { Fish } \\
\text { Rem.* }\end{array}$ & $\begin{array}{l}\text { Parasitic } \\
\text { Amimals }\end{array}$ & Crustacea & $\begin{array}{l}\text { Other } \\
\text { Animals }\end{array}$ \\
\hline 1184 & $\begin{array}{l}\text { terna } \\
\text { fuscata }\end{array}$ & mains & 3 & & + & & & \\
\hline 1186 & $\begin{array}{l}\text { Gygis } \\
\text { alba }\end{array}$ & 1 & & 17 & + & & & \\
\hline 1187 & $\begin{array}{l}\text { Stejna } \\
\text { Junata }\end{array}$ & & & & - & & & \\
\hline 1188 & $\begin{array}{l}\text { uffinus } \\
\text { psciti- } \\
\text { cus }\end{array}$ & & 211 & & - & & & \\
\hline 1189 & $\begin{array}{l}\text { Puifinus } \\
\text { pacifi- } \\
\text { cus }\end{array}$ & & 57 & & + & & & \\
\hline 1190 & $\begin{array}{l}\text { pufininus } \\
\text { pacifi- } \\
\text { cus }\end{array}$ & & 61 & & - & & & \\
\hline 1191 & bulweria & & & & - & & & \\
\hline 1192 & $\begin{array}{l}\text { Bulweria } \\
\text { bulwerii }\end{array}$ & & 4 & & + & & & \\
\hline 1193 & $\begin{array}{l}\text { ufinus } \\
\text { pacifi- } \\
\text { cus }\end{array}$ & & 133 & & - & & & 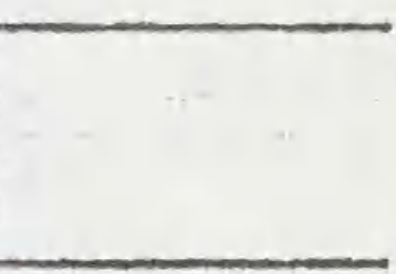 \\
\hline 11.94 & $\begin{array}{l}\text { Sterna } \\
\text { fuscata }\end{array}$ & ne- & & & - & & & \\
\hline 1195 & $\begin{array}{l}\text { puininus } \\
\text { pacifi- } \\
\text { cus }\end{array}$ & 1 & & & - & & & \\
\hline 1296 & $\begin{array}{l}\text { Sterna } \\
\text { Suscata }\end{array}$ & & & 1 & - & & & \\
\hline 1197 & $\begin{array}{l}\text { Bulweria } \\
\text { bulwerit }\end{array}$ & $\begin{array}{l}\text { yyes } \\
(5)\end{array}$ & & & + & & & \\
\hline 1198 & $\begin{array}{l}\text { Sterna } \\
\text { fuscata. }\end{array}$ & & & & - & & & \\
\hline 1199 & $\begin{array}{l}\text { Bulweria } \\
\text { bulwerif }\end{array}$ & & & 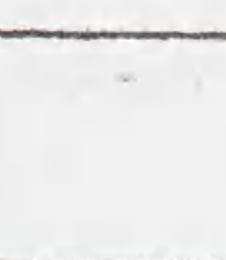 & - & & & \\
\hline 1200 & $\begin{array}{l}\text { Sterna } \\
\text { fuscata }\end{array}$ & & & & - & & & \\
\hline 1201 & $\begin{array}{l}\text { sterini } \\
\text { cerules } \\
\text { Puffinus }\end{array}$ & & & & - & 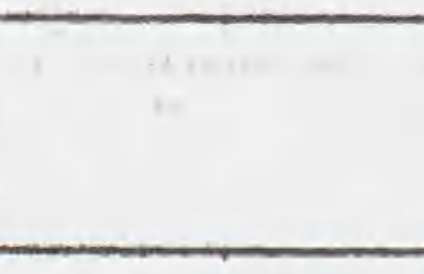 & ? & $\begin{array}{l}\text { Halobates } \\
\text { (38) }\end{array}$ \\
\hline 1202 & $\begin{array}{l}\text { Puffinus } \\
\text { pacifi- } \\
\text { cus }\end{array}$ & 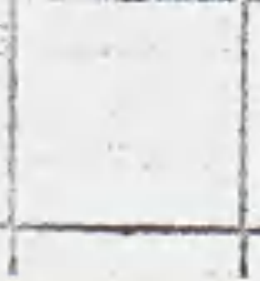 & 31 & & - & & & \\
\hline
\end{tabular}


* pish remains indicated

$b y+o r$ - sign
SOSC REF. NO. 204

POBSP NO. 8

\begin{tabular}{|c|c|c|c|c|c|c|c|c|}
\hline $\mathrm{Sta} \mathrm{NO}$ & Species 1 & Squida & $\begin{array}{l}\text { Squid } \\
\text { bealss }\end{array}$ & Fish & $\begin{array}{l}\text { Fish } \\
\text { Rem.* }\end{array}$ & $\begin{array}{l}\text { Parasitic } \\
\text { Animals }\end{array}$ & Crustices & $\begin{array}{l}\text { Other } \\
\text { Animals }\end{array}$ \\
\hline 1203 & $\begin{array}{l}\text { pacifi- } \\
\text { cus }\end{array}$ & & 84 & & - & & & \\
\hline $120 \%$ & $\begin{array}{l}\text { Puffinus } \\
\text { pacifi- } \\
\text { cus }\end{array}$ & & 42 & & - & & & \\
\hline 1205 & $\begin{array}{l}\text { Puffinus } \\
\text { weifi- } \\
\text { cus }\end{array}$ & & 85 & & + & $\begin{array}{l}\text { Nematodes } \\
\text { (2) }\end{array}$ & & \\
\hline 1206 & $\begin{array}{l}\text { Puffinm } \\
\text { pacifi- } \\
\text { cus }\end{array}$ & & 81 & & + & & & \\
\hline 1207 & $\begin{array}{l}\text { Anous } \\
\text { stolidus }\end{array}$ & & & & - & & & \\
\hline 5238 & $\begin{array}{l}\text { Ducula } \\
\text { pacifica }\end{array}$ & & & & - & & & \\
\hline 5239 & $\begin{array}{l}\text { Ducula } \\
\text { pacifical }\end{array}$ & & & & - & & & \\
\hline 524,0 & $\begin{array}{l}\text { Ducula } \\
\text { pacifica }\end{array}$ & & & & - & & & \\
\hline 20262 & $\begin{array}{l}\text { Sterna } \\
\text { fuscrta. }\end{array}$ & & & & - & & & 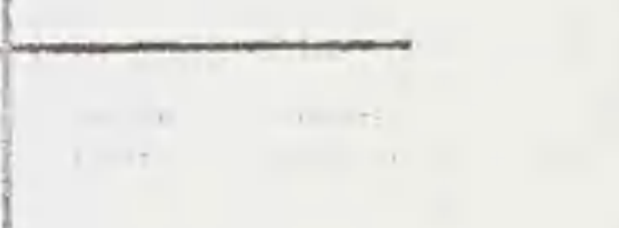 \\
\hline 20274 & $\begin{array}{l}\text { werna } \\
\text { Puscata }\end{array}$ & & & & - & & & \\
\hline 20279 & $\begin{array}{l}\text { Stern: } \\
\text { fuscat? }\end{array}$ & & & & - & & & \\
\hline 20230 & $\begin{array}{l}\text { Stema } \\
\text { fuccata }\end{array}$ & & & & + & & & \\
\hline 20234 & $\begin{array}{l}\text { renaria } \\
\text { inter- } \\
\text { pres }\end{array}$ & & & & - & & & \\
\hline 20295 & $\begin{array}{l}\text { Pluvialis } \\
\text { dominica }\end{array}$ & & & & - & & Remains & $\begin{array}{l}\text { Forams } \\
\text { (3I) }\end{array}$ \\
\hline 20309 & $\begin{array}{l}\text { Brolia } \\
\text { melanotds }\end{array}$ & & & & - & & & $\begin{array}{l}\text { Forams }(7) \\
\text { Insect remains }\end{array}$ \\
\hline 20310 & $\begin{array}{l}\text { Erolia } \\
\text { melanoto }\end{array}$ & & & & - & & & Forams (12) \\
\hline 20311 & $\begin{array}{l}\text { rolia } \\
\text { melano- } \\
\text { tos }\end{array}$ & & & & - & & - & Forams (13) \\
\hline 20318 & $\begin{array}{l}\text { Arenaria } \\
\text { interpres }\end{array}$ & & $=$ & & - & & $\ldots$ & $\begin{array}{l}\text { Forams (56) } \\
\text { Gastropods (3) } \\
\text { Insect Inva (1) }\end{array}$ \\
\hline
\end{tabular}


*tsir temains indicated

by + of $-5 i g n$ p. 7 of 10

SOSC REF. NO. 204 POBSP NNO. 8

\begin{tabular}{|c|c|c|c|c|c|c|c|c|}
\hline Sta No. & Species & Squid & $\begin{array}{l}\text { Squid } \\
\text { beaks }\end{array}$ & Fish & $\begin{array}{l}\text { Fish } \\
\text { Rem.* }\end{array}$ & $\begin{array}{l}\text { Parasitic } \\
\text { Animals }\end{array}$ & Crustacea & $\begin{array}{l}\text { Other } \\
\text { Animals }\end{array}$ \\
\hline 20323 & $\begin{array}{l}\text { Arenaris } \\
\text { interpre }\end{array}$ & S & & & - & & $\begin{array}{r}\text { Remains } \\
\text { (I) }\end{array}$ & $\begin{array}{l}\text { Gastropod ( } \\
\text { Forams }(51)\end{array}$ \\
\hline 20331 & $\begin{array}{l}\text { Luviatic } \\
\text { dominica. }\end{array}$ & & & & - & & $\begin{array}{c}\text { Remains } \\
\text { (I) }\end{array}$ & Forams (2) \\
\hline 20359 & $\begin{array}{l}\text { Ptero- } \\
\text { Aroma } \\
\text { Svpoleud }\end{array}$ & & & & - & & & \\
\hline 20363 & $\begin{array}{l}\text { Sula } \\
\text { leuco- } \\
\text { raster }\end{array}$ & & & & - & & & \\
\hline 20364 & $\begin{array}{l}\text { Anous } \\
\text { stolidus }\end{array}$ & & & & - & & & \\
\hline 20365 & $\begin{array}{l}\text { Dero- } \\
\text { droma } \\
\text { iynoleut }\end{array}$ & & 24 & & 7 & & & $\begin{array}{l}\text { Pyebalis }(5 \\
\text { Pelecypods } \\
\text { (10) }\end{array}$ \\
\hline 20366 & $\begin{array}{l}\text { Hinno- } \\
\text { dromus } \\
\text { species }\end{array}$ & & & & - & & & $\begin{array}{c}\text { Gastropods } \\
(I I)\end{array}$ \\
\hline 20367 & $\begin{array}{l}\text { Detero- } \\
\text { scelus } \\
\text { Ancanum } \\
\end{array}$ & & & & - & $\begin{array}{l}\text { Tapeworm } \\
\text { (1) }\end{array}$ & & $\begin{array}{r}\text { Forams } \\
\text { (3) }\end{array}$ \\
\hline 20369 & $\begin{array}{l}\text { holia } \\
\text { melino-- } \\
\text { tos }\end{array}$ & & & & - & Lice (2) & & $\begin{array}{l}\text { Orams (10) } \\
\text { Gastropods } \\
(6)\end{array}$ \\
\hline 20370 & $\begin{array}{l}\text { molia } \\
\text { melano- } \\
\text { tos }\end{array}$ & & & & - & & & $\begin{array}{l}\text { Gastropod(1) } \\
\text { Insect Grub } \\
(75)\end{array}$ \\
\hline 20371 & $\begin{array}{l}\text { urcus } \\
\text { cyaneus }\end{array}$ & & & & - & & & \\
\hline 20373 & $\begin{array}{l}\text { Pegata } \\
\text { minor }\end{array}$ & & 56 & & - & $\begin{array}{c}\text { Nematodes } \\
\text { (2) }\end{array}$ & & \\
\hline 20375 & $\begin{array}{l}\text { Arenarie } \\
\text { inter- } \\
\text { pres }\end{array}$ & & & & - & & $\begin{array}{l}\text { Remains } \\
\text { (1) }\end{array}$ & $\begin{array}{l}\text { Ants }(28) \\
\text { Ant remains } \\
\text { Forams }(9)\end{array}$ \\
\hline 20379 & $\begin{array}{l}\text { Ands } \\
\text { acuta }\end{array}$ & & & & - & & & $\begin{array}{c}\text { Foraminifera } \\
(14 I)\end{array}$ \\
\hline 20380 & $\begin{array}{l}\text { Pluvial- } \\
\text { is } \\
\text { dominicel }\end{array}$ & & & & - & & & $\begin{array}{c}\text { Pelecypods } \\
(2)\end{array}$ \\
\hline 20381 & $\begin{array}{l}\text { Sula } \\
\text { sula }\end{array}$ & & 88 & & - & & & \\
\hline 20382 & $\begin{array}{l}\text { Larus } \\
\text { species }\end{array}$ & & & & + & & & \\
\hline 20383 & $\begin{array}{l}\text { Mareca } \\
\text { penelope }\end{array}$ & & & & - & $\begin{array}{c}\text { Nematodes } \\
(8)\end{array}$ & & $\overline{x+2}$ \\
\hline
\end{tabular}


* Sta remains inaticated $b y+o r-b i g n$

\begin{tabular}{|c|c|c|c|c|c|c|c|c|}
\hline Sta No. & Spectes & Squia & $\begin{array}{l}\text { Squia } \\
\text { beaks }\end{array}$ & Fish & $\begin{array}{l}\text { Fish } \\
\text { Rem.* }\end{array}$ & $\begin{array}{l}\text { Parasitic } \\
\text { Animals }\end{array}$ & Crustacea & $\begin{array}{l}\text { Other } \\
\text { Animals } \\
\end{array}$ \\
\hline 20403 & Enrus & & & 3 & + & $\begin{array}{c}\text { Nematode } \\
\text { (I) }\end{array}$ & & Amphipod \\
\hline 201,05 & $\begin{array}{l}\text { Rissa } \\
\text { tridacty } \\
z\end{array}$ & & & & - & & & \\
\hline 204.44 & $\begin{array}{l}\text { Larus } \\
\text { species }\end{array}$ & & & 2 & + & & & \\
\hline 20415 & $\begin{array}{l}\text { Larus } \\
\text { argenta- } \\
\text { bus }\end{array}$ & Re- & 2 & & + & $\begin{array}{l}\text { Nematodes } \\
(4)\end{array}$ & & $\begin{array}{c}\text { Gastropod } \\
\text { (1) } \\
\text { Eoram (62) }\end{array}$ \\
\hline 204,41 & Diomedey & mains & 25 & & - & & & \\
\hline 20445 & $\begin{array}{l}\text { Oceano- } \\
\text { droma } \\
\text { markhami }\end{array}$ & & & & - & $\begin{array}{l}\text { Nematodes } \\
\text { (8) }\end{array}$ & & \\
\hline 20446 & $\begin{array}{l}\text { Faleo } \\
\text { peregri- } \\
\text { nus }\end{array}$ & & & & - & & & \\
\hline 20447 & $\begin{array}{l}\text { Lamus } \\
\text { species }\end{array}$ & $m i$ & & & + & $\begin{array}{l}\text { Nematodes } \\
(4)\end{array}$ & $\begin{array}{r}5 \\
4 \quad 4 \\
\end{array}$ & $\begin{array}{r}\text { Gastropods } \\
\begin{array}{r}(41) \\
\text { Eorems }(675)\end{array}\end{array}$ \\
\hline 20448 & $\begin{array}{l}\text { Larus } \\
\text { occiden- } \\
\text { talis }\end{array}$ & 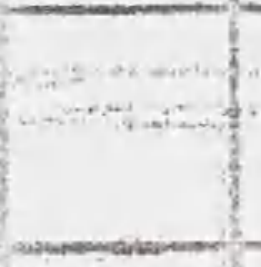 & & n....... & - & a & Remains & ans \\
\hline 20449 & $\begin{array}{l}\text { Lmus } \\
\text { species }\end{array}$ & & & & + & & Remains & $\begin{array}{l}\text { Forams }(576) \\
\text { Pelecypod }(1) \\
\text { Gestroneds }(100)\end{array}$ \\
\hline 20450 & $\begin{array}{l}\text { Larus } \\
\text { species }\end{array}$ & & & & + & $\begin{array}{l}\text { Nematode } \\
\text { (I) }\end{array}$ & Remains & Forams (16) \\
\hline 20452 & $\begin{array}{l}\text { Phaethon } \\
\text { rubri- } \\
\text { cauda }\end{array}$ & mains & & 1 & + & & & \\
\hline 20453 & $\begin{array}{l}\text { Anas } \\
\text { acuta }\end{array}$ & & & & - & & & \\
\hline 20454 & $\begin{array}{l}\text { Anas } \\
\text { acuta }\end{array}$ & & & & - & & & $\begin{array}{c}\text { Forams } \\
\left(21_{+}\right) \\
\end{array}$ \\
\hline 20456 & $\begin{array}{l}\text { Gygis } \\
\text { alba }\end{array}$ & & 3 & & - & & $\ldots$ & \\
\hline 20461 & $\left\{\begin{array}{l}\text { Larus } \\
\text { species }\end{array}\right.$ & & & & + & & & Foram (I) \\
\hline 25021 & $\begin{array}{l}\text { Anous } \\
\text { stolidu }\end{array}$ & $\begin{array}{l}\text { Re- } \\
\text { main }\end{array}$ & & - & + & $\theta$ & $\overline{\ldots \ldots \ldots+n}$ & \\
\hline 25029 & $\begin{array}{l}\text { Sterna } \\
\text { fuscata }\end{array}$ & $\mid \cdots \cdots$ & & & - & 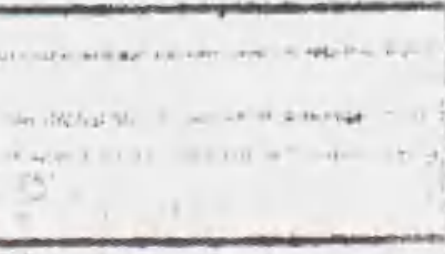 & & $\begin{array}{r} \\
\end{array}$ \\
\hline
\end{tabular}


His remains indicated

$b y+0 z-s i g n$

SOSC RET. NO. 204

POBSP NO. 8

\begin{tabular}{|c|c|c|c|c|c|c|c|c|}
\hline Sta $\mathrm{NO}$ & Species & squid & $\begin{array}{l}\text { Squid } \\
\text { beaks }\end{array}$ & Fish & $\begin{array}{l}\text { Fish } \\
\text { Rem.* }\end{array}$ & $\begin{array}{l}\text { Parsositic } \\
\text { Animals }\end{array}$ & Crustacea & $\begin{array}{l}\text { Other } \\
\text { Animals }\end{array}$ \\
\hline 25034 & $\begin{array}{l}\text { Anous } \\
\text { stolidu }\end{array}$ & & & & + & & & \\
\hline 25036 & $\mid \begin{array}{l}\text { Stema } \\
\text { fuccata }\end{array}$ & & & & - & & & \\
\hline 25038 & $\begin{array}{l}\text { Stema } \\
\text { fuscata }\end{array}$ & & & & - & & & \\
\hline 25039 & $\begin{array}{l}\text { Sterna } \\
\text { fuscata }\end{array}$ & & & & - & & & \\
\hline 30036 & $\begin{array}{l}\text { Procel- } \\
\text { sterna } \\
\text { serulea }\end{array}$ & $\cdots$ & & & - & & & \\
\hline 30079 & $\begin{array}{l}\text { Jetero- } \\
\text { celus } \\
\text { incanim }\end{array}$ & mains & & 23 & + & & $\operatorname{Crabs}(2)$ & 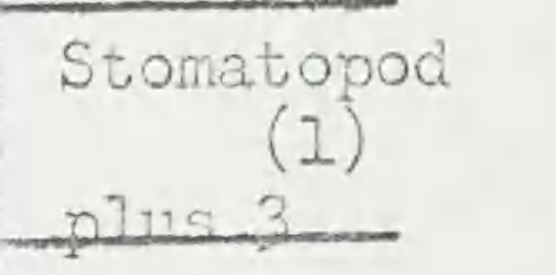 \\
\hline 30084 & $\begin{array}{l}\text { Sterna } \\
\text { fuscata }\end{array}$ & $\begin{array}{l}\text { Re- } \\
\text { mains }\end{array}$ & 3 & & - & & & \\
\hline 30099 & $\begin{array}{l}\text { Arenarid } \\
\text { inter- } \\
\text { ores } \\
\end{array}$ & & $\because$ & & - & - & Remains: & $\begin{array}{l}\text { Gastropods }(79) \\
\text { Forams (31) }\end{array}$ \\
\hline 30103 & $\begin{array}{l}\text { Arenarid } \\
\text { inter- } \\
\text { pres }\end{array}$ & (n) & and & & - & 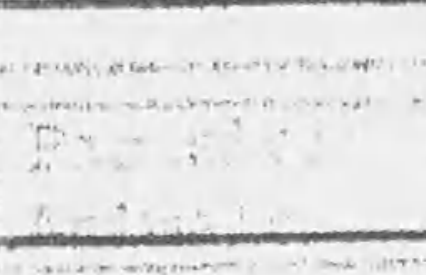 & $\begin{array}{l}\text { Isopads (2) } \\
\text { Remains }\end{array}$ & $\begin{array}{l}\text { Castropods (59) } \\
\text { Forams }(7) \\
\text { Insects (3) }\end{array}$ \\
\hline 30111 & $\begin{array}{l}\text { Sterna } \\
\text { sumatrar }\end{array}$ & a & & & + & & & \\
\hline 30290 & $\begin{array}{l}\text { Hetero- } \\
\text { scelus } \\
\text { incanum }\end{array}$ & & $\overline{x+600}$ & & - & & $\begin{array}{l}\text { Crabs }(5)^{-} \\
\text {Remains }\end{array}$ & $\begin{array}{l}\text { Arachnida- } \\
\text { Remains }\end{array}$ \\
\hline 30292 & $\begin{array}{l}\text { Sterna } \\
\text { suma- } \\
\text { trana }\end{array}$ & & & & + & & & \\
\hline 30293 & $\begin{array}{l}\text { Stema } \\
\text { suma- } \\
+ \text { trana }\end{array}$ & & & & + & & & $\begin{array}{c}\text { Gastropod(1) } \\
\text { Foraminifera } \\
(131)\end{array}$ \\
\hline 30295 & $\begin{array}{l}\text { Sterna } \\
\text { suma- } \\
\text { treng } \\
\end{array}$ & & & 1 & + & & & \\
\hline 30296 & $\begin{array}{l}\text { Sterna- } \\
\text { suma- } \\
\text { terana }\end{array}$ & & & 1 & + & & con & $\bar{\square}$ \\
\hline $30290 \%$ & $\begin{array}{l}\text { Sterna } \\
\text { suma- } \\
\text { trana }\end{array}$ & & & & + & & $\ldots$ & $\ldots$ \\
\hline 30313 & $\begin{array}{l}\text { Hetero- } \\
\text { scelus } \\
\text { incanum }\end{array}$ & & & & - & & 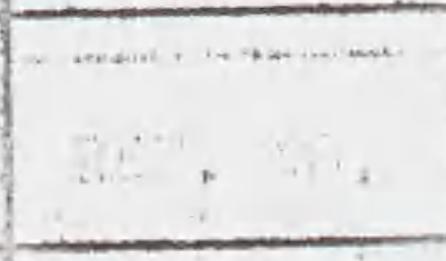 & 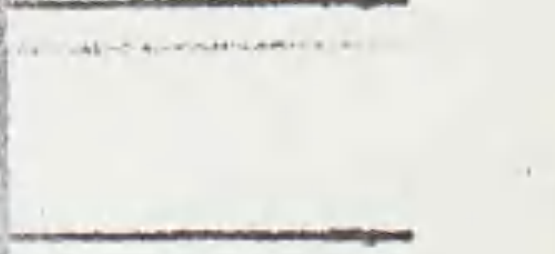 \\
\hline 110002 & $\begin{array}{l}\text { Hetero- } \\
\text { scelus } \\
\text { ins anmm }\end{array}$ & & $+\cdots+\cdots$ & & - & & 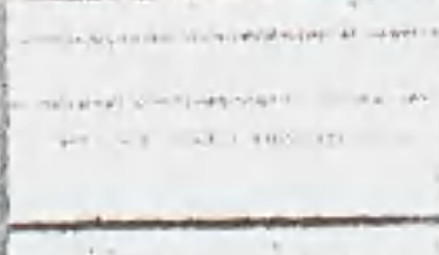 & Foraminifera \\
\hline
\end{tabular}


* Fish remains indicated

by + or - sign
SOSC REF. NO.

POBSP NO.

p. 10 of 10

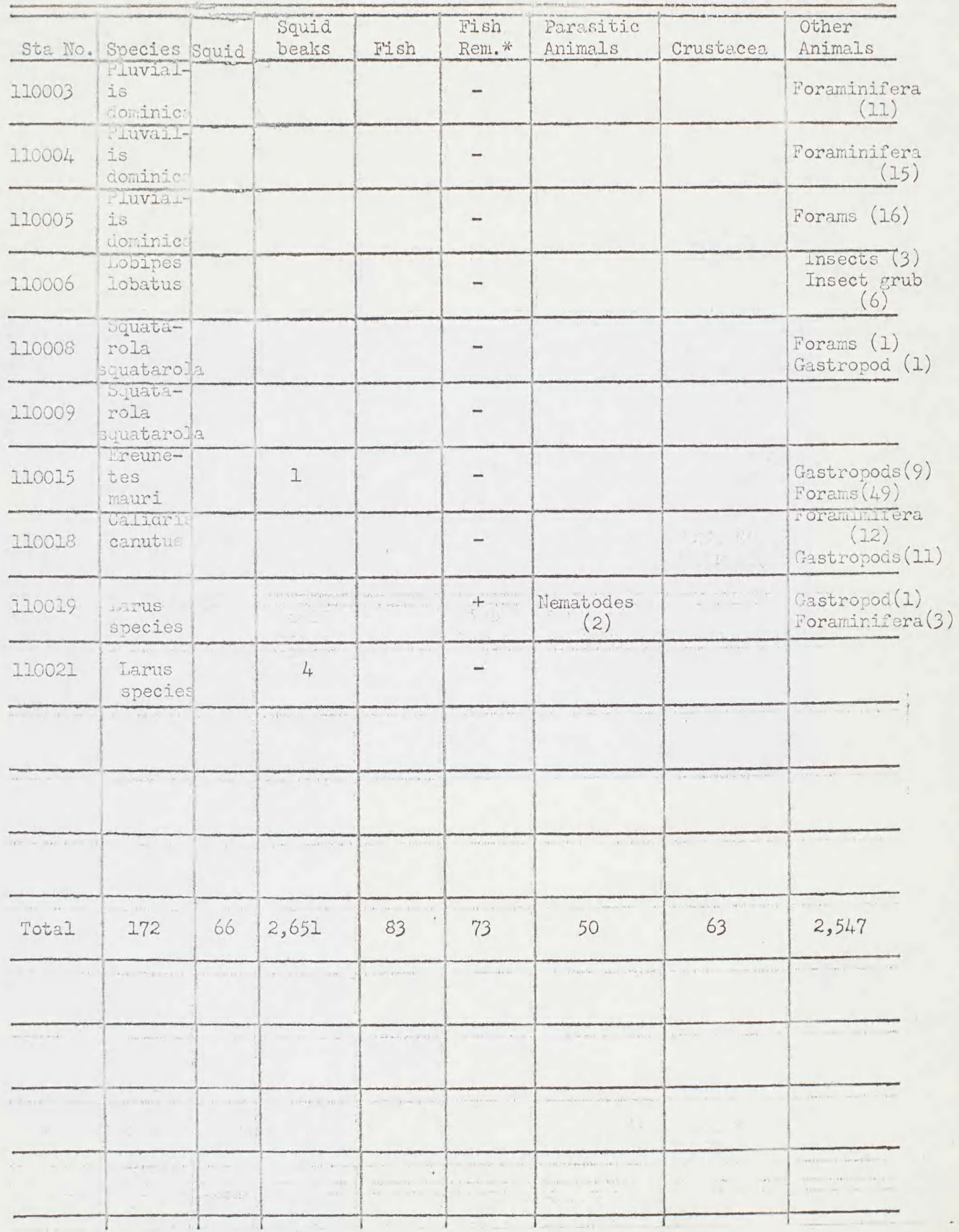

